The Institute has attempted to obtain the best original copy available for filming. Features of this copy which may be bibliographically unique,

which may alter any of the images in the reproduction, or which may significantly change the usual method of filming, ere checked below.

Coloured covers/

Couverture de couleur

Covers damaged/

Couverture endommagée

Covers restored and/or laminated/

Couverture restaurée et/ou pelliculée

Cover title missing/

Le titre de couverture manque

Coloured maps/

Cartes géographiques en couleur

Coloured ink (i.e. other than blue or black)/

Encre de couleur (i.e. autre que bleue ou noire)

Coloured plates and/or illustrations/

Planches et/ ou illustrations en couleur

Bound with other material/

Relié avec d'autres documents

Tight binding may cause shadows or distortion along interior margin/

La reliure serrée peut causer de l'ombre ou de la distortion le long de la marge intérieure

Blank leaves added during restoration may appear within the text. Whenever possible, these have been omitted from filming/

II se peut que certaines pages blanches ajoutées lors d'une restauration apparaissent dans le texte, mais, lorsque cela était possible, ces pages n'ont pas été filmées.

Additional comments:/

Commentaires supplémentaires:
L'Institut a microfilmé le meilleur exemplaire qu'il lui a été possible de se procurer. Les détails de cet exemplaire qui sont peut-être uniques du point de vue bibliographique, qui peuvent modifier une image reproduite, ou qui peuvent exiger une modification dans la méthode normale de filmage sont indiqués ci-dessous.

Coloured pages/

Pages de couleur

Pages damaged/

Pages endommagées

Pages restored and/or laminated/

Pages restaurées et/ou pelliculées

Pages discoloured, stained or foxed/

Pages décolorées, tachetées ou piquées

Pages detached/

Pages détachées

Showthrough/

Transparence

Quality of print varies/

Qualité inégale de l'impression'

Includes supplementary material/

Comprend du matériel supplémentaire

Only edition available/

Seule édition disponible

Pages wholly or partially obscured by errata slips, tissues, utc., have been refilmed to ensure the best possible image/

Les pages totalement ou partiellement obscurcies par un feuillet d'errata, une pelure. etc., ont été filmées à nouveau de façon à obtenir la meilleure image possible.

This item is filmed at the reduction ratio checked below/

Ce document est filmé au taux de réduction indiqué ci-dessous.

$10 x$

$14 x$

$18 \times$

$22 x$

$26 x$

$30 x$

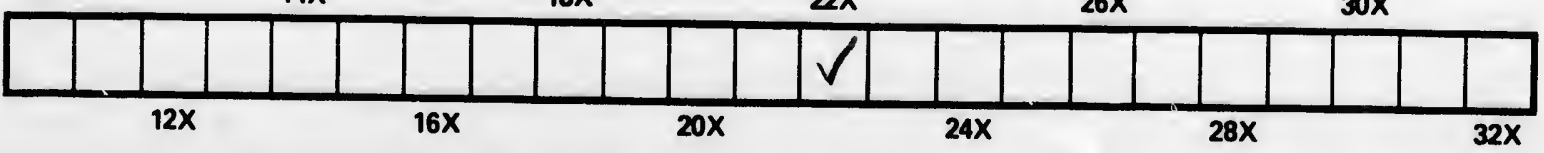


The copy filmed here has been reproduced thanks to the generosity of:

\section{National Library of Canada}

The images appearing here are the best quality possible considering the condition and legibility of the original copy and in keeping with the filming contract specifications.

Original copies in printed paper covers are filmed beginning with the front cover and ending on the last pags with a printed or illustrated impres. sion, or the back cover when appropriate. All other original copies are filmed beginning on the first page with a printed or illustrated impression, and ending on the last page with a printed or iflustrated impression.

The last recorded frame on each microfiche shall contain the symbol $\rightarrow$ (meaning "CONTINUED"), or the symbol $\nabla$ (meaning "END"), whichever applies.

Maps, plates, charts, etc., may be filmed at different reduction ratios. Those too large to be entirely included in one exposure are filmed beginning in the upper left hand corner, left to right and top to bottom, as many frames as required. The following diagrams illustrate the mothod:
L'exemplaire filmé fut reproduit grâce à la générosité de:

Bibliothéque nationale du Canada

Les imagus suivantes ont été reproduites avec le plus grand soin, compte tenu de la condition $\epsilon t$ de la netteté de l'exemplaire filmé, et en conformité avec les conditions du contrat de filmage.

Les exsmplaires originaux dont lá couverture en papier est imprimée sont filmés en commençant par le premier plat at en terminant soit par la dernière page qui comporte une empreinte d'impression ou d'illustration, soit par le second plat, selon le cas. Tous les autres exemplaires originaux sont filmés en commençant par la premiere page qui comporte une empreinte c inpression ou d'illustration et en terminant par iá Iarnière page qui comporte une telle empreinte.

Un des symboles suivants apparaîtra sur la derniére image de chaque microfiche, selon le cas: le symbole $\rightarrow$ signifie "A SUIVRE", le symboie $\nabla$ signifie "FIN".

Les cartes, planches, tableaux, etc., peuvent être filmés à des taux de réduction différents. Lorsque le document est trop grand pour être reproduit en un seul cliché, il est filmé à partir de l'angle supérieur gauche, de gauche a droite, et de haut en bas, en prenant le nombre d'imag as nécessaire. Les diagrammes suivants illustrent la méthode.
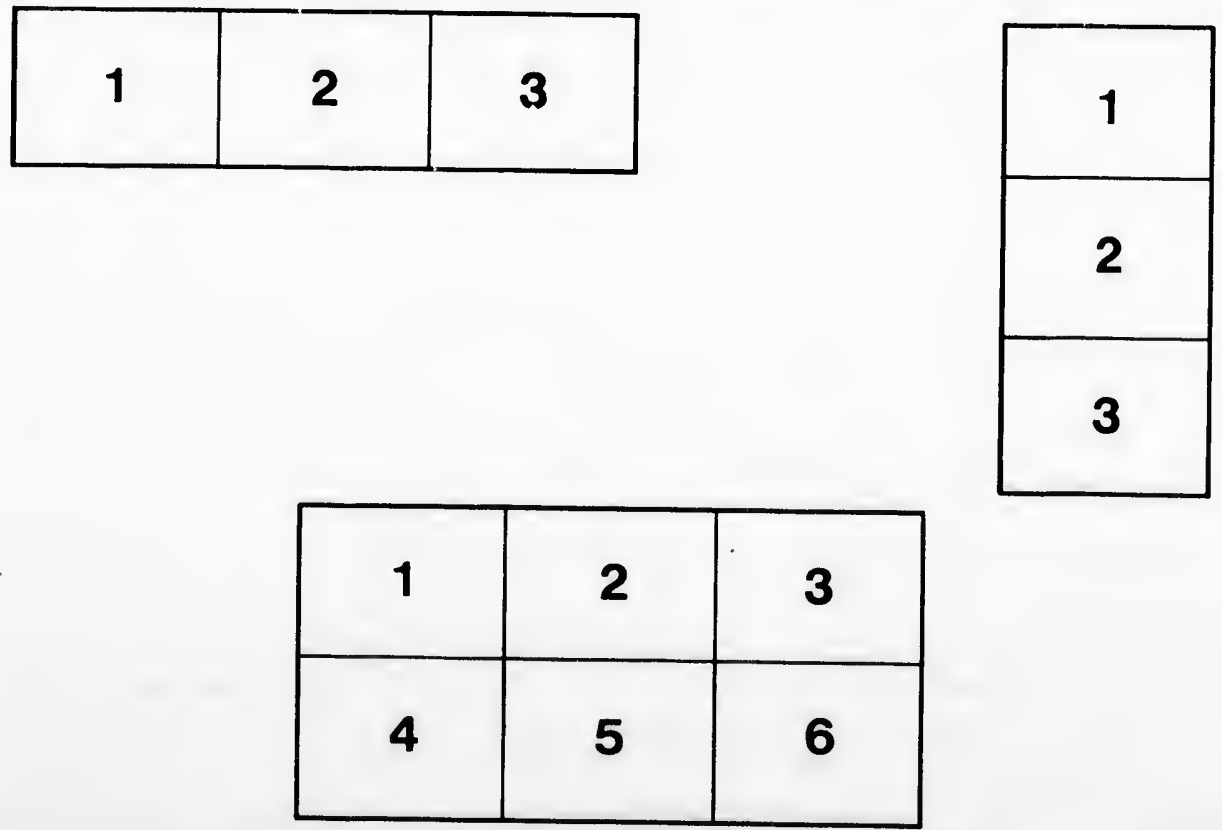


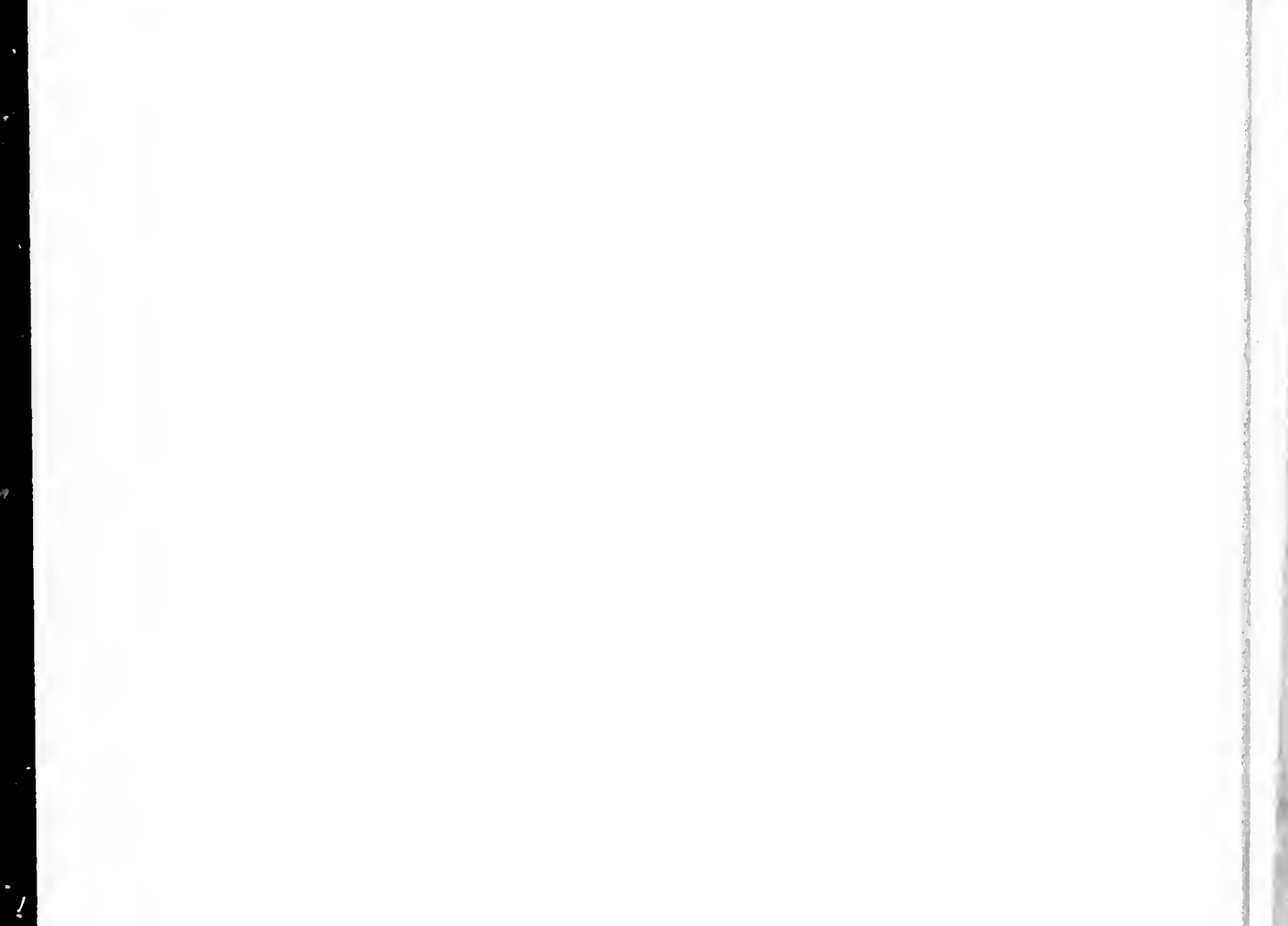





\section{ECOLE D'ARPEMTAGE RT DE GÉNIE FORESTIE}

\section{LE \\ BASSIN MERIDIONAI,}

DE LA

B A I E D'H U DSON

CHAPITRE I

SITUATION-LIMITES-ÉTENDUE-APERCQU GENERAI

Nons comprenons sous cette désignation la partie du bassin de la baie d'Hudson située au sud du soixantième degré de latitude.

Cette immense région a pour limite orientale le cent-vingtième degré de longitude. Sa limite onest et nord-onest est formée par la ligue de partage des eaux séparant le bassin du Mackenzie de celni de la baie d'Hudson et courant à peu près dans la direction suivante: à partir de la montagne du Cheval, vers $54^{\circ}$ de latitude et $113^{\circ}$ de longitude, elle se prolonge entre le nord et le nord-ouest jusqu'an portage Methy en passant au sud-est du lac de la Biche. De ce point elle féchit au sud pour traverser le lac Wollaston et elle remonte ensuite itu nord pour atteindre le soixantième degré de latitude dans les environs de son point d'intersection par le cent cinquième méridien. La limite méridionale de cet immense territoire s'étend depuis la montague du Cheval jusqu'au cent vingtième degré de longitude, en passant par les points qui suireut: au sud de la rivière au Castor et du lac Vert, où elle décrit une courbe vers le sud pour remonter au nordest jusqu'au portage de la Grenouille, près de la vallée du fleure Churchill, vers $103^{\circ}$ de longitude. Ici elle dévie à droite et court au sud-est jusqu'à la décharge du lac Winnipeg, en passant par la Pointe-à-la-Monsse et à l'ouest du lac Jack pour atteindre le lac Favorable, qui se trouve sur la ligne de fatte séparant les eaux qui coulent directement vers la baie d'Hudson de celles qui se jettent dans le lac Winnipeg. Cette ligne passe ensuite cntre le lac Seul et le lac Saint-Joseph puis se prolonge au sud-est jusqu'à l'intersection dú $50 \mathrm{e}$ parallèle par le 90e méridien. En cet endroit, elle prend la dirsction du levant et décrit un $\mathbf{S}$ pour contourner le lac Népigon au nord, le lac Long au 


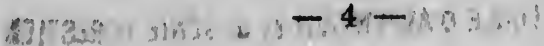

sud, les sources de la rivière Pic au septentrion, les lacs Missinaibi et Kenogamisi-formant les sources de la rivière Albany-au midi, après quoi elle reprend sa course régulière, en déviant un pen à gauche pour passer eutre le lac Témiscamingue et le lac Abittibi pour courir daus la mêmo direction jusque dans le voisinage du 75 e degré de longitude. Enfin, elle remonte au nord, contourmant le lac Mistassini au sud-est, jusqu'au 70e degré de longitude, qui forme la limite orientale de cet immense territoire. (*)

L'aire comprise dans ces limites forme une étendue d'environ 750,000 milles carrés, ou plus de trois fois l'étendue de la province de Québec. Sa plus grande longueur dépasse sept cents milles. La configuration générale de ce pays représente a peu près le développement extérieur d'un cône tronqué, avec la base vers le sud, où elle cescend, dans la partie est, jusqu'an delà de $47^{\circ}$ de la latitade. Ce territoire occupe à peu près la même position astronomique que le grand plateau central de la Russie d'Europe, peuplé par plus de vingt millions d'habitants. En retranchant de l'étendue totale, l'aire occupée par ia partie inférieure de la baie d'Hudson et la baie James, dont les eaux couvrent à peu près 250,000 milles carrés, il reste pour les terres plus de 500,000 milles, ou au deld de deux fois et demie la superficie de la province de Québec.

A raison de sa position astronomique et de la nature du sol qu'il renferme, ce vaste territoire n'est pas susceptible de culture dans toutes ses parties, et à ce point de vue, il faut défalquer de l'étendue des terres plus du tiers de leur surperficie totale pour compenser la lisière contigüe aux euux de la baie d'Hudson et qui, au point de vue agricole, n'a pratiquement ancune valeur. Dans la partie orientale, ce désavantage est racheté par l'existence de riches mines do fer et d'autres muerais qui j urront fournir matière à des opérutions considérables, du moment qu'on aura mis ce pays en communication régulière avec les régions habitées qui se trouvent plus au sud.

Avant d'entrer dans les détails, dennons un aperçu général de ce grand bassin hydrographique de la baie d'Hudson, qui est en général si peu ou si mal connu.

Dans l'esprit de beaucoup de gens, - la baie d'Hudson n'est qu'une partie des régions polaires. C'est une erreur et une erreur grave, puis: qu'aucune partie de ce grand bassin ne pénètre dans le cercle arctigne et que la latitude de son éxtrémité méridionale so trouve mème an sud du méridien de Londres. Cette erreur provient de ce que l'on n'a généralement qu'une idée très incomplète de cette grande méditerrannée du Canada.

En comprenant la baie James, qui en forme le prolongement vers

(*) Ces bornes, ninsi que tous les renseignements relatifs a l'ótendue de ce territoire,sont pris sur la Carte indiquant 'a limite nord des principaux arbres forestiers du Canada, préparée par le Dr Robert Bell, et sur la Carte d'une partie du territoire du Nord-Ouest,pour accompagner le hupport du Ministre de l'intérieur, 31 déc. 1880.

(") Ces détails sont pris dans une excellente étude publiée par le Dr Bell, dans le Rapport, de la Commission Géologique du Canada pour 1879-80. 
le sud, la baie d'Hudson mesurè à peu près mille milles de longueiur et plus de six cents milles de largeur à son extrimiténord. Sou étendue totale approche 500,000 carrés, ou plus de la moitió de celle de la Méditerrannée. Elle est environnée par la terre de tous les cotés, excepté celui de l'est, où elle a par le détroit d'Hudson son débouchó dans l'océan Atlantique.

La baie d'Hudson forme le grand bassin par lequel s'écoulent les eanx de la partie centrale de l'Amérique du Nord. Du côté de l'ouest,ce bassin est pour ainsi dire prolongé par celui du lac Winnipeg, qui recueille les eaux venant de cette direction pour les écouler ensuite par la rivièNelson dans la baie d'Hudson. Au sud, les rivières qui se jettent dans la baie James ont leurs sources à peu de distance du lac Huron et la rivière Albany prend même une partie de ses eaux à moins de viugtcinq milles au nord du lac Supérieur. En y conprenant le bassin sécondaire du lac Winnipeg, l'étendue du teritoire dont les eaux coulent dans la baie d'Hudson mesure une largeur de 2,100, de l'est à l'ouest et nne longueur de 1,500 du nord an sud, ce qui forme une superficie totale d'a peu près $2,000,000$ de milles carrés.

Sur une grande partie de cette immense région, le climat est tempéré et si le sol n'est pas partout susceptible de culture, il offre cependant de grandes étendues qui n'attendent que le travail de l'hom. me pour produire d'excellentes moissons.

La baie James, qui forme pour ainsi dire le centre de la région que nous nous propcsons d'étudier spécialement, commence du côté de l'ouest au cap Henriette-Marie, et du côté de l'est au cap Jones, puis s'átend vers le sud jusqu'à une distance de 350 milles. Sa largeur moyenne est d'environ 150 'milles. On désigne généralement sous le nom d'Eest Main la partie orientale de la baie James et de la baie d'Hudson. Le long de cette dernière baie, entre le cap Jones et le cap Dufferin, qui forme l'extrémité du promontoire de Portland, le littoral est haut et escarpé, atteignant sou vent une élévation de près de 2000 pieds au-dessus du niveau de la mer. La partie sud-ouest de la baie d'Hudson et le pays situé à l'onest de la baie James sont bas et généralement unis et les rivages assèchent $a$ une grande distance à mer basse.

Au sud, la baie d'Hudson et la baie James reçoivent les eaux de plusieurs grandes rivières, dont quelques-unes sont assez profondes pour être navigables par des bateaux à vapeur d'un moyen tirant d'eau. Ainsi la rivière Albany offre à partir de son embouchure une ligne de navigation d'environ 250 milles de longueur. Dans les grandes eaux, la rivière Moose pourrait aussi fournir, à partir de son embouchure, une autre ligne de navigation d'une centaine de milles. Enfin la rivière Nelson peut être remontée par les plus gros bateaux à rapeur jusqu'à soixante et dix ou quatre-vingts milles de la mer et la rivière Hayes et deux de ses tributaires peuvent donner en sus cent quarante milles de navigation a la vapeur.

Sur le côté ouest de la baie d'Hudson, l'estuaire de la rivière Nel- 
son est à peu près le seul port de mer praticable, à l'état naturel. Cet estuaire assèche a marée basse ; mais il est treversé au inilieu par un chonal qui remonte jusqu'au point où la marée cesse de se foire sentir et donne une profondeur moyenne de deux brasses dans les basses eaux. Il serait très facile et peu coûteux de faire disparaltre les hantsfonds qui soulèvent le lit de ls rivière et de la rendre parfaitement navigable jusqu'd une distance de quatre-vingts milles de la mer. Outre ce chenal, il y en a un autre plus profond qui avance dans la baie et forme le havre qu'on désigue sous le nom de York Roads, ou rivière du Nord.

L'accès de la rivière Churchill est plus facile, à raison de sa plus grande profondeur; mais cet avantage est plus que contre-balancé par le peu d'étendue de ce havre, qui est eutouré par des falaises rocheuses d'une grande hauteur et n'offre aux gros narires qu'il pourrait recevoir qu'un ancrage difficile et peu sûr.

Eufiu la baie James offre par elle-rnème une ligue de navigation de plus de 350 milles, avec une profondeur d'eau suffisante pour recevoir les navires propres à desservir le commerce de ces régions.

Au point de rue géologique, la région que nous étudions appartient aux formations laurentiennes. Des roches cambro-silurionnes, reposant presque horizontalement sur les couches lanrentiennes, forment une grande bordure irrégulière au côté sud-ouest de la baie, et dans les va!lées des grandes rivières, ces couches cambro-silurieunes s'étendent jusqu'à deux cents milles dans les terres, où elles sont recourertes pardes formations dévonienues d'une grande étendue. Les couches métalliféres se rencontrent principalement $d u$ côté est, au nord $d u$ cap Jones.

Les ressources du pays qui environne la baie James et la baie d'Hudsou sont nombreuses et variées : elles comprennent la pêche ì la baleine, la clıasse au marsouin, au morse, à l'ours blanc, au loup marin et à d'autres animaux fournissant l'huile, l'ivoire et d'autres produits ; mais les plus précieuses de ces ressourcesse trouvent dans le sol agricole, les forêts et les mines. Au sud et au sud-onest de la baie James,sous la même latitude que le Devonshire et le Cornouailles, il y a une grande aire dont le sol est bon et le climat favorable aux opérations agricoles. Ces conditious se rencontrent pareillement dans, une lisière située à l'est de la baie James. Dans la région égoutée par les rivières Nelson et Churchill, entre le lac Winnipeg et la mer, la moitié orientale n'est pas susceptible de culture ; mais la moitié ouest pourrait être en général cultivée avec profit. Dans la région de ia baie James, il y a beaucoup de bois susceptible d'exportation et qui deviendra avant longtemps l'objet d'un commerce considérable. Leś principales essences sont le pin ronge, le pin blanc, le pin résineux, l'epinette noire et blanche, le sapin, le cèdre blanc et le boulean. Les rivières qui se jettent dans la baie offrent les jlus grandes facilités à l'exploitation de ces forêts.

Mais les mines constituent de beaucoup la plus grande ressource de la région avoisinant la baic d'Hudson. Il y a des gisements considérables de fer carbonaté daus la vallée de la rivière Mattagami, 
qui formo la principale branch de la rivière Moose. Les tles qai bordent la côte de l'Liast-Main renferment des minérais de for spathique en quantité inépuisable et la galène existe dans les environs du golfo de Richmond et de la petite rivière à la Baleine. On a aussi trouvé de l'or, de l'argent et du nolybdène sur les côtes de l'East-Main. Il y a du lignite dans la vallée de la rivière Missinaibi, au gypse dans celle de la rivière Moose et du pétrole sur les bords de la rivière Abittibi. Enfin, il y dans cette région de l'anthracite et plusieurs bonnes variétés de pierre d'ornementation, sans compter les pierres à bâtir et ane foule d'uutres matières minérales qui prendront de la valeur a mesure que le pays s'établira.

Te! est, en résumé, lo pays que nous nous proposons d'étudier un peu en détail dans catte esquisse.

\section{CHAPITRE II}

\section{HYDROGRAPHIE-RIVIלRES ET LACS}

L'hydrographie du territoire qui nous occupe peut se diviser en trois sections : celle de l'est, celle du sur et cell h de l'onest.

La première de ces sections est traverséo de l'est à l'ouest par les rivières qui prennent leurs eaux dans le plateau central $d u$ Labrador et les écoulent dars la partie orientale de la baie James et de la baie d'Hudson. A partir du nord, on trouve en allant vers le sud la rivière Nastapoka, la rivière du lac à l'Eau Claire, qui se jette dans le golfe de Richmond, ainsi que la petite et la grande rivière d la Baleine. T'outes ces rivières ont leurs sources dans de grands lacs situés entre les 70 e et 75e degrés de longitude, au nord du 55e degré de latitude. Elles sillonnent un pays inculte et qui n'a de valeur qu'd raison des mines qu'il renferme sur les bords de la baie d'Hudson. Les forèts de cette région se composent de sapins, de peupliers, de trembles, do bouleaux et d'épinettes blanches qui ne valent rien comme bois de commerce.

Plus au sud, ou rencontre la Grande-Rivière, qui se jette dans la baie du fort George, l'East Main et la rivière Rupert. Par la longueur de son cours, qui traverse deux degrés de longitude, l'East-Main est à peu près la plus grande rivière de cette région, d̀ laquelle elle a donné son nom. La rivière Rupert ramasse ses eaux par trois branches supérieures, dont une sort du lac Mistassini. Elle traverse une contrée plus unis et bien moins élevée que celle à travers laquelle coulent les rivières situées plus au nord. Les forêts qui bordent son cours inférieur renferment des bois susceptibles d'exploitation mercantile et une partie des terrains qui avoisinent son embouchure pourraient faire un bon pays de pâturages, ainsi que le pronve l'existence du bétail que la compagnie de la baie d'Hudson entretient depuis bien long. temps dans cet endroit. La même observation s'applique à la vallêe 
inférieure de la rivière Eupert, qui traverse un pays eucore plus avantageux.

La rivière Notavay à sa principale souzce dans le lac Abatagomaw, situé sur la hauteur des terres et à moins d'un demi-mille du lac $\mathrm{Nl}_{\mathrm{t}}$ koubau, d'où sort la rivière Chamouchouan, qui se jette dans le lac SaintJeau. Lu lac Abatagomaw est élevé de 1339 pieds au-dessus du niveau de la mer et situé dans un pays plât et convert de bois de bonnes dimensions. A partir du lac, la rivière coule de l'est à l'onest jusqu'à l'extrémité sud-est de lo baie James, où elle a son embouchure. La rivière Hannah à son embouchure' un peu plus au sud et prend ses eaux à peu de distance des sources de la Gatineau et de la rivière des Dutaouais. Sa vallée ressemble sous tous les rapports à celle de la Notaway: mais traverse une région douée d'un meilieur climat.

La rivière Moose, avec ses importants tributaires, se jettc dans la partie la plus méridionale de la baie James. En allant de l'est à l'ouest, ces tributaires sont la rivière $A b i t t i b i$, la rivière Mattag $a m i$ et la rivière Missinaïbi.

La rivière A3ittibi a sa principale source dans un petit lac situé à peu de distance de la hauteur des terres et à environ trente milles du lac Des-Quinze, dans la vallée de l'Outaouais. En sortant de ce lac, la rivière continue sa course ou nord et traverse le lac Matawasojign, qui a huit milles de longueur et n'est éloigné que de deux milles de la hauteur des terres. 'A partir de ce dernier lac, la rivière parcourt une distance de onze milles, où elle forme quatre rapides, pour atteindre le lac Agotawekami, qui est long de six milles et entouré d'un pays passablement accidenté. Quelquers chalnes plus bas que ce lac, la rivière reçoit un affluent venant du sud-ouest et suit la même direction jusqu'au lac Abittibi, qui est à neuf milles plus loin.

Le lac Abittibi se compose de deux nappes distinctes. Celle du hant, qui reçoit les eaur de la rivière à son extrémité nord-est, s'étend de l'est à l'onest. Sa lougueur est de trente-trois milles et sa largeur varie de deux à huit inilles, excepté vers le milieu, où deux grandes baies situées vis-à-vis l'une de l'autre portent la larger $r$ du lac à environ dix-sept milles. A son extrémité sud-est, cette partie du lac communique par une espèce de détroit de deux milles de longuear avec l'autre partie, qui est presque circulaire, dont la largeur varie de quinze à vingt milles et dont la longreur est d'environ douze milles, ce qui forme avec la gorge qui unit les denx parties vne longueur totale de quarante-sept milles pour tout le lac. Le rivage est partout fort irrégulier et les deux parties du lac sont parsemées d'un très grand nombre d'lles. La surface de ce lac est élevée de $\$ 57$ audessus du niveau de la mer.

A la sortie du lac, d̀ son extrémité sud-ouest, la rivière Abitibi fait deux milles au sud-ouest puis fléchit à l'ouest et à cinq milles plus loin forme la chute Couckiching,'qui a cinquante pieds de hauteur. A partir àe cette chate, le cours de la rivière suit à peut près la direction suivante : 
1. De la chute an confluent de la rivière Noire...

2. De la rivióre Noire nu ruisseande la Machuire.

N. $89^{\circ} \mathrm{E} 45+$ milles

3 De la Jachoire ì la riviùre Frédéric.

4. De la rivière Fródéric au rapide Sexiant...

N. $13 \circ 054$ "

5. De ce rapicle is son contiuent.

$\mathrm{V}_{1} 83^{\circ} \mathrm{F} 10$ \%

S. $5 \circ \mathrm{E}$ hin 4

Entre la décharga du lac Abittibi et le confluent de la rivière du même ucm avec li rivière Moose, la distance, en suivant le cours de la ri, ière, est de 186 milles et la pente de 807 pieds, puisqu'à son confluent le lit de li Moose est élevé de einquante pieds au-dessus du nireau de la mer. Avant de se jeter dans la rivière Monse, l'Abittibi forme plusieurs chenaux séparés par des tles, et dont le cours est fort rapide, sur une distance d'une dizaine de milles.

A partir de son embouchure et en remontant, la rivière Abittibi conle à traters un sol uni at réconvert de diluvium. Lus bords, qui ne sont pas élevés, se composent de glaise souvent recouverte par un peu le sable et de gravier ainsi que par une terre brune grasse. Plus haut, jusqu'à une centaine de milles de son embouchure, la vallée est étroite et borçée par des collines dont la hauteur varie de 50 à 200 pieds. Au delà de cette distance, le pays environnant est uni, excepté dans le roisinage de la sortie du lac, où l'on trouve des deux côtés des collines de 80 à 120 pieds de hauteur.

La rivière Mattagami, qui forme la branche centrale de la Moose, coule à l'ouest de l'Abittibi. Elle a sa source daus le lac Kenogamisi et se jette daus la Missinaïbi après avoir décrit un cours de $170 \frac{1}{2}$ milles, divisé comme suit:

- 1 Du lac Kenogamisi au coude formé par le confluent $\mathrm{du}$ premier ruissenu............

2 De ce ruisseau à un autre faisant un coude au N. E

3 De ce deuxième ruisseau à un troisième faisant un coude au sud-ouest................

4 De ce troisième ruisseau au rapide de l'Ours, ou Mackwa Povitik ..............

7 Du rapide do l'Ours an pied du Long-Por-

6 Du pied du Long Portage à la Missi-

Direction Distances

N. E. 12 milles

N. 3 “

O. 5 "

N. 66 “ naibi........................................

N. $18^{\circ}$ O. 45
N, $42^{\circ}$ E. $\frac{39 \frac{1}{2}}{170 \frac{1}{2}} "$

Entre le lac Kenogamisi et le Long-Portage, la largeur moyenne de la Mattagami est d'environ cinq chałnes. Dans cette espace, des in. tervalles d'eau tranquille alternent arec de petits rapides; mais audessous du Lnng-Poriage, le lit de la rivière est peu profoud, son cours très rapide et sa largeur varie de dix à quinze chaines. 
Depuis la hauteur des terres jusqu'au Long-Portage, la Mattagami coule à travers un plateau de formation huronienne et laurentienne dont la hauteur moyenne est d'environ 1,200 pieds an-dessus du niveau de la mer. La surface du pays est un peu accidentée; mais les jnégálités du sol excèdent rarement un ou deux cents pieds. Les hauteurs sent rocheuses; mais les intervalles sont rernplis par des lacs ou des marais reposant sur un sous-sol de gravier, recourert à la surface par une couche plus ou moins épaisse de terre végétale. A partir du Long-Portage, la rivière coule à travers uu pays plat tet un sol reposant sur des roches primitives. Les bords, qui sont généralement bas, se composent d'un terrain de glaise dans lequel on rencontre du gravier et des cailloux à certains endroits. Près de l'embouchure de la rivière, les fles et la terre ferme se composent d'une terre d'alluvion très propre à la culture.

La rivière Missinaïbi forme la branche ouest de la rivière Moose, dans laquelle elle écoule ses eaux après les avoir versées dans la Mattagami. Elle a sa source dans le lac Missinaïbi et coule du sud au uord en décrivant une courbe rers l'ouest. Depuis son embouchure jus-. qu'au Grand-Coude, distance d'euviron quatre-vingt-un milles, la direction générale de son cours est à peu près sud $67^{\circ}$ ouest. Sur tout ce parcours, le courant est très fort, quelquefois intercepté par de petits rapides et la largeur de la rivière varie de huit à dix channes. Les bords sont généralement bas ; ils s'élèvent en beancoup d'endroits à trente et cinquante pieds de hauteur et sont formés par une glase bleue, grisâtre reposant sur une couche de glaise bleue. A plusieurs places, ces bandes de glaise bleue renferment des gisernents de lignite que l'on rencontre sur un long parcours.

La rivière Moose, qui n'est que la continuation des trois rivières que nous venons de décrire, se jette dans l'extrémité sud de la baie James. Entre le confluent de la Missinaibi et la mer, distance de quarante-six milles, le ccurs de la rivière Moose est nord $52^{\circ}$ est. Il est tranquille et à peu près libre de tout ubstacle. La région traversée par la rivière est unie et repose sur des formations primitives qui n'ont subi aucun boulerersement. Les bords de la rivière sont peu élevés et généralement formés par des conches de terre et de glaise renfermant du gravier et quelques cailloux. Au-dessus de ces bords, le sol est sec et supporte une deuxième crue de peupliers, de bouleaux et de conifères. En gaguant l'intérieur, le terrain est marécageux; mais les tles et la terre ferme qui avoisinent l'embonchure de la rivière sont formées par une terre d'alluvion on ne peut plus propre à la culture, notamment l'lle sur laquelle est bâti le fort Moose, à dix-huit milles de la mer.

La rivière Albany a ses deux principales sources dans le lac Long et et dans le lac Saint-Joseph. La branche est, qui sort du du lac Long et atteint le bras principal au fort Henley, coule presque parallèlement à la rivière Missinaibi et reçoit pltisieurs affluents considérables. Cette rivière est désignée sous le nom de Kenogami ou rivière des Anglais. 
L'extrémité sud du lac Long, ou Kenogami, est à environ vingt et un milles au nord du lac Supérieur, en droite ligne. A partir de son extrémité supérieure et sur une distance de hriit milles et demi, la la direction générale du lac est franc nord et sa largeur varie de deux à quarante chaines. Depuis ces huit milles et demi, jusqu'à sa décharge dans la rivière Kenogami, la course du lac va à peu près en droiteligne, nord $30^{\circ} \mathrm{est}$, de sorte que la course générale de tout le lac entre ses deux extrémités est à peu près nord-nord-est. Sa largeur moyenne dans la dernière section, qui a quarante-six milles de longueur, est de cent quatre chaines, on un peu plus d'un mille et quart. La contrée qui aroisine l'extrémité sud est accidentée, montagneuse et n'offre que des roches de gneiss presque nues. La plus haute de ces moutagnes, située à deux mille's et demi à l'ouest, est élevée de 540 pieds au-dessus du nivau du law. En allant au nord, la région avoisisinante devient moins accidentée et d partir du milieu de la longneur $\mathrm{du}$ lac, elle est comparativement unie.

En sortant de ce lac, la rivière Kénogami coule sur une distance de deux milles à travers des marais où la compagnie de la baie d'Hudson fait couper le fourrage nécessaire à l'alimentation du bétail qu'elle entretient au poste du lac Long. Dans les premiers neuf milles, à partir du lac, la rivière court nord $10^{\circ}$ est, forme deux petits rapides et reçoit les eaux de deux affluents. Vers le sixième mille, la largeur de la rivière n'est que de deux chaines, mais elle va toujours en augmentant, et à quatre-vingt-dix milles du lac, elle a une moyenne de dix à douze chaines. Au bout des neuf milles mentionnés plus haut, la rivière fait un détonr, courant nord $86^{\circ}$ est sur une distance de huit milles, pour entrer à angle droit dans le lac des Pins, qui a sept milles et demi de longueur et un mille et demi de largeur.' Plus bas, elle traverse le lac du Brus et le lac Ka-ples-a-Watan, reçoit plusieurs affluents et forme une dizaine de petits rapides qui, avec les antres portages, donnent au cours de la rivière une déclivité totale de 282 pieds depnis le lac Long jusqu'au furt Henley.

Sur tout son cours, la rivière Kenogami traverse une région unie. Dans la partie supérieure, on rencontre à certaines distances des bosses de syénite et de gneiss; mais ces petites inégalités disparaissent plus bas que le lac des Pins et toute la surface du pays environnant devient uniformément uni. Des assises ou des terrasses de terre brune grasse et de gravier s'élèvent sur les bords de la rivière de dix à quarante pieds. Sur le haut de ces terrasses et à une certaine distance de la rivière, le sol a paru très bon dans presque tous les endroits où il a été examiné. La forêt se compose en graude partie d'épinettes, de sapins, de cèdres blancs, de tamaracs, de bouleaux et de trembles. Les plus grosses épinettes blanches et rouges mesurent de cinq à six pieds de circonférence à cinq pieds au-dessus $\mathrm{du}$ sol ; mais le diamètre moyen de ces arbres est d'environ dix-huit pouces.

La branche ouest, ou branche principale de la rivière Albany, a plu- 
sieurs sources et prend ses eaux dans le grand plateau qui s'étend du sud-est au nord entre la hauteur des terres, au nordd-onest du lac Nipigon, et le lac au Loup-lle-mer. Le plus grand de ces lacs est le lac Saint-Joseph, qui mestre plus de 50 milles de longtur. Presque toutes ces branches supérienres se jettent dans le cours principal di la rivière Albany avant l'entrée de cette dernière dans le lac Abazotiki/chebaw. En sortant de ce lac, la rivière court sud-est sur une distance de huit milles, pour atteindre la tête du lac Makokebatan. Dans ce parcours, elle franchit sept rapides et sa largenr varie entre une dizaine de chaines et un demi-mille. Le lac Makokebatan est presque droit ; il a onze milles de longueur et un mille et demi de largeur. Les bords de ce lac sont formés par de petits cailloux et des bancs de sable, et le pays qui l'environne est tellement uni, qu'en regardant de l'uno des eztrémités du lac, on ne peut pas découvrir la terre à l'autre. La rivière Albany en sort par deux canaux qui se réunissent au lac Moosewaki, à vingt milles plus loin. Entre le lac Moosewaki et la chute Martin, distance de vinit milles, le cours de la rivière est intercepté par une multitude d'iles et de rapides.

Depuis la tête du lac Makokebatan jusqu'à la chute Martin, qui en est éloignée d'environ einquante-six milles, il y $\cap$ quinze rapid's ou cascades. La plus grande de ces cascades est la chule de Ka-gi-ami, qui a quarante-einq pieds de hauteur. Sur toute eette distance, la course générale de la rivière est nord $70^{\circ}$ est. De chaque côté, le sol \& st à peu près uni et paraft être de bonne qualité.

En bas de la chute Martin; la largeur, la profondeur et la vélocité du courant de la rivière devienuent plus uniformes. Dans les cent vingt milles compris entre la chute et le confluent de la rivière Kenogami, sa largeur varie de vingt à trente chafnes, sa profondeur de cinq à vingt nieds et la vitesse du crurant est d'environ trois milles à l'heure.

Entre la chute Martin et le confluent de la rivière Ogoki, l'Albany décrit une courbe dont le sommet se prolonge à trente-sept milles vers le nord, puis court à l'est sur une distance de vingt et un milles 't dévie au sud-est pour arriver jusqu'à la rivière Kenogami, à soixante et un milles plus loin. A partir du confluent de la Kenogami, la rivière Albany court au nord-est jusqu'à son embouchure dans la baie James. Depuis son embouchure jusqu'à la chute Martin, qui en est éloigné de 250 milles, la rivière Albany est narigable pour de forts bateaux à vapeur. Sur tout ce parcours, le terrain qui avoisine ses bords est uni et se compose de glaise, de marne et de détritus de coquillages dans lesquels on découvre à peine quclques petits cailloux. En s rançant dans l'intérieur, le terrain est un peu marécageux, mais pourrait être facilement égoutté et transformé en un excellent.sol agricrile.

Los autras rivières de la section sud sont l'Attawapiscat et l'Equan,qui coulent nest à l'est et se jettent dans la baie James, la Sever. at coulent au nord et débouchent dans la baie d'Hudson. 
La Severn est une rivière considérable. Elle a sa source la plus éloignée dans le lac Favorable et un cours de plus de trois cents milles de longueur. Elle traverse une région unie et la vallée de son cours supérieur renferm + de grandes étendues de terrain $q$ qui, à raison de la qualité du sol et du climat, sont suceptibles de culture.

La section ouest est traversée par les rivières Hayes, Nelson et Churchill.

La rivière Hayes a plusieurs branches. La principale sort du lac Molson, ou Winnipegosis, et sous le nom de rivière Franklin court vers le nord-est en traversant lo lac Robinson, le lac des Pins et le lac du Vent. Les cascades Robinson, les plus grandes qu'il y ait sur toute la rivière, foiment pour ainsi dire la décharge du lac. Polir éviter ces vascades, qui ont une déclivité de 45 pieds, les voyageurs out ouvert sur les bords de la rivière un chemin de portage passant sur un terrain de glaise grise. Cette glaise grise forme partout le sol de cette région. Quatorze milles plus bas que les cascades, la rivière traverse le lac des Pins et un peu plus loin le lac du Vent. A partir de ce dernier lac, la rivière fuit un détour de quatre milles vers le nord-ouest, saute une chute de six pieds et traverse un marais pour entrer dans la baie ou le bras sud-ouest du lac Oxford. Ce lac a trente milles de longueur et il est large de huit à neuf milles. Il écoule ses eaux dans la rivière à la Truite, qui les déverse daus le lac du Genou après leur avoir fait parcourir une distance d'une douzaine de milles. Le lac du Genou a quarante milles de longueur et six de largeur dans la partie inférienre. Il est onvironné par un terrain plat et un sol de glaise grise et de terre brune d'une grande fertilité. Le lac du Genou se décharge par Ja rivière Jack dans le lac des Savannes et à partir de ce dernier lac la rivière abandonne sa cor rse vers le nord-est pour se diriger à peu près au nord jusqu'à son embouchure dans la baie d'Hudson.

Entre le lac des Savannes et le confluent de la rivière aux Reuards, le cours principal est désigné sous le nom de rivière de la Montagne, fit de ce dernier point an confluent de la Shamattawa, e'est la rivière d'Acier. Enfin, depuis la Shamattawa jusqu'à la baie d'Hudson, c'est la rivière Hayes. Entre la montagne Brassy et la rivière aux Renards, le cours de la ririère a jusqu'à trois quarts de mille de largeur et il est intercepté par plusieurs rapides. Le dernier de ces rapides, qui se troure à 109 milles du fort York, tire son nom d'un massif de gneiss situé à peu de distance de la rivière et qui s'élèric à 292 pieds au-dessus du plateau environnant. A partir du confluent de la Shainattawa, la largeur de la rivière Hayes augmente de dix chatnes, à vingt-quatre milles de son embouchure, jusqu'à un mille en face du fort York.

Le cours supérieur de la rivière Hayes, jusqu'anx cascades Robinson, est parfaitement navigable. Ces quarante-neuf milles de navigation pourraient facilement se rattacher aux trente-sept milles qu'il y a depuis la sortie du lac Winuipeg jusqu'à la chûte de la rivière de I'Est, ce qui ferait une ligue de quatre-vingt-six milles' en tout, à 
travers une région qui peut fournir de très bons terrains à la colonisation.

La rivière Nelson égontte tout le bassin du lac Winnipeg et le plateau situé entre la rivière Hayes et la rivière Churchill. Sa course géuérale est vers le nord-est, en décrivant une légère courbe au nordonest. Elle renferme dans la première partie de son cours une foule d'sles et de lacs qui forment comme un labyrinthe à travers lequel l'éuorme volume de ses eaux se précipite avec une impétuosité extraordinaire.

La région qu'elle traverse dans les deux premiers tiers de son cours es unie el va ell s'abaissaut vers la mer à raison d'environ deux pieds au mille. Sur les cent premiers milles, à partir du grand lac Playgreen, la Nelson ne coule pas dans une vallée, mais se ramifie par de nombreux canaux à travers une région d'une étendue considérable. Cette tendance à se répaudre en de nombreux canaux manque dans toutes les grandes rivières de la région que nous étudions, mais elle caractérise tout particulièrement la rivière Nelson. Ses rameaux se réunissent parfois, mais ce n'est que pour se diviser de nouveau et former un réseau de rivières entourant des lles àe toutes formes et de toutes grandeurs. Ces canaux sont accidentés par des chutes et des rapides d'autant plus nombreux qu'ils n'ont généralement que peu de déclivité. Tous ces rapides rendent la navigation impossible jusqu'au lac Sipi-Wesk, à environ quatre-vingts milles de la ehute de la rivière de l'Est ; mais entre le lac Sipi-Wesk et le lac Fendu, éloignés d'à peu près cen milles l'un de l'autre, il n'y a pas le moindre obstacle à la navigation et de forts bateaux à vapeur pourraient parcourir cette distance arec la plus grande facilité.

A partir du lac Fendu, où elle reunit à peu près toutes les eaux de ses principaux tributaires, la rivière Nelson coule jusqu'à la baie d'Hudson dans un lit profond et moins accidenté. Un peu plus bas que le lac Fendu, la largeur de la rivière est d'à peu près un quart de mille et elle angmente toujours en allant vers la mer, où elle excède six milles. A partir de son embouchure, la Nelson est narigable sur une distance de plus de eent-milles, en sorte que toutes ses parties navigables forment environ 200 milles, on les deux tiers de la longueur totale de son cours.

La source la plus éloignée de la rivière Churchill est la tête de la rivière aux Castors, qui n'est séparée que par un portage de deux milles du lac de la Biche. (") De cette première source au lac de l'yle à la Crosse, à l'eau haute du moin's, la rivière aux Castors est navigable pour des canots d'écorce. Si l'eau pouvait se maintenir à cette hauteur, cette rivière serait d'autant plus avantageuse qu'elle traverse un pays en grande partie propre à la colouisation. La plaine au milieu de laquelle elle coule est toute couverte de lacs magnifiques où

(*) Mgr Taché, Esquisse sur le Nord-Ouest de l' Amérique. 
abonde le poisson. Les ramifications qu'elle forme à ses scurces relient un grand nombre de ces petits lacs. Parmi ses afluents, on remarque ensuite la rivière du Nord. Le lac Froid, qui reccit les eaux du lac des Outardes, commence la série des lacs de la Truite, du Détroit et de la Poule d'Eau qui, avec la rivière qui porte ce dernier nom, formeut une route parallèle à la rivière aux Castors et qui est souvent suivie pour passer de l'lle à la Crosse au lac la Biche.

La rivière aux Castors se décharge dans'le lac de l'ile à la Crosse, un des principaux anneaux de la chatne de lacs connue sous le nom de rivière Churehill. Remontons à une autre source de cette dernière, le lac des Iles, alimenté par des rivières qui viennent du pays des Montagnais. Cu lac enroie une partie de ses eaux au lac des Roches, qui les remet au lac des Eufs, où elles attendent celles du lac de lạ Loche, qui 'st ordinairement considéré comme la tête de la rivière Churchill. En laissant le lac de la Loche, on entre dans celui du Bœuf, long de 40 milles, et qu'en richit la rivière du mème nom.

Le lac de l'lle à la Crosse, long d'environ 60 milles, réunit les eanx de toutes les sources de la rivière Churchill et les confie ensuite à la rivière la Puise, qui se jette dans le lac Primeau. Après avoir franchi plusieurs cascades, les eaux de la Churchill arrivent à l'embouchure de la rivière Rapide, affluent du sud qui, par le lac du Lièvre, décharge le grand lac Laronge et les antres qui s'y rattachent.

la partie de la rivière Churchill que nous venons de décrire, longueur de 300 à 400 milles, n'est navigable que pour des légères embarcations ; mais de l'extrémité sud du lac de l'yle à la Crosse jusqu'à l'embouchure de la rivière de la Loche; distance d'environ 120 milles, il n'y a pas d'obtacl.'s à la navigation.

Un peu plus bas que le portage du fort de la Truite, on aperçoit l'embonchure de la rivière Caribnu, qui sort du lac du même nom, Deer Lake. Ce lac est l'un des plus grands de l'Amérique et ne mesure pas moins de 150 à 200 milles de longueur. Il reçoit les eaux du lac des Brochets et du lac Wollaston, qui a aussi une de ses issues vers le lac Athabaska.

A partir de l'embouchnre de la rivière Caribou, la Churchill continue son cours sinuenx et accidenté vers le nord-est et forme par l'expansion de ses eaux le lac Grenville, le lac South Indian, ou Grand Lac, et le lac North Indian, qui est moins grand que les deux précédents. Sur tout ce parcours, la largeur de la rivière Churchill est d'environ un mille. La région qu'elle traverse est rocheuse, dépourvue de végétation ou couverte par une forêt d'arbres de petites dimensions. In certains endroits, on rencontre des épinettes rouges d'une assez grande hauteur, mais d'un faible diamètre.

Telles sont les principales rivières ou fleuves qui se jettent dans le bassin méridional de la baie d'Hudson. La conformation même de la contrée qu'elles égouttent indique assez que leur cours doit être accidenté, intercepté par des cascades et des rapides, puis impropre à la navigation. Toute cette région est à peu près entourée par le ver- 
sant nord des Laurentides, composé cie terrains de différents âges et donnant prise à l'action de l'eau, lorsque cette dernière sort des roches huroniennes ou laureniiennes pour frayer son chemin à travers les terrains glaiseux qui avoisinent la baie James et la baie d'Hudson.

Comme on a pu le voir par la discription des principales rivières, les lacs se trouvent tous dans la partie la plus élevée de cette contrée et surtont dans la partie la plus rapprochée de la ligne de paitage des eanx entre le versant rord et le versant sud des Laurentides. Ceux de la région ouest sont les plus grands et les plus nombreux ; ils ocupent presque le quart du grand plateau dans lequel les rivières Nelson et Churchill out leurs sourees. Ces grands laes sont tous remplis de poisson et pourraient devenir plus tard la source d'une exploitation importantu, lorsque la civilisation sura rapproché ces régions incultes des grands centres de commerce et de population.

\section{CHAPITRE III}

ESQUISSE GEOIOGIQUE-RELIEFS DU SOL-MONTAGNES-TERRAINS

CULTIVABI.ES-LEUR ETFNDUE

Au point de rne géologique, la région que nons étudions se troure comprise dans les grandes formations laurentiennes du Canada. Des roches cambro-siluriennes. reposant presque horizontalement sur les couches laurentiennes, forment une bordure irrégulière autour de la partie sud-onest de la baie, et dans les vallées de quelques-unes des rivières qui riennent du sud-ouest, ces terrains cambro-siluriens s'avancent jusqu'à cent et même deux ccnt milles dans l'intérieur. des terres. Au sud et à l'onest de la baie James, ces couches cambro-siluriennes sont recouvertes par des terrains dévoniens qui forment une aire considérable. Ces terrains cambro-siluriens forment un pays uni, à peine accidenté par de rares dykes de trap. La surface du sol se compose de matièrus diluviennes, recouvrant nne couche de glaise cailloutense. Du côté est, la chatne d'iles qui hordent la côte jusqu'à 300 milles au nord du cap Jones, ainsi que la terre ferme daus le voisinäge du golfe Richmond, est formée par des roches sédimentaires ot des strates rolcaniques, qui n'ont subi presqu'ancune altération et paraissent appartenir à l'âge cámbrien inférieur.

Les terrains d'origine cristalline forment une espèce de bande qui contourne l'extrémité méridionale da bassin de la baie d'Hudson. Cette bande a son plus grund développement à ses deux extrémités, 
¡l'est at à l'ouest. Vers le miliou, ello est. rétrécio par les terrains diluviens qui atteignent presquo les bords du lac Supérieur, nu sud de la baie fames. Un pou plus ì l'ouest, lo groupe laurentien est dominé par les formations huromnienmes, qui forment nuo lisière se prolongeant au nord jusqu'aux environs de la rivière Nelson, eu passant par lo lac Oxford. Dans cetto région, comme dans la province de Québec, le systìme laurentien est loin deformer un sol aride et sans valeur. Au contraire, il est entrecoupó par des espaces de terrains diluviens et d'immenses étendues de sol glaisenx et très fertile, ainsi que cela so roit surtont dans les environs du lac Mistassini et dn lac $\Lambda$ bittibi et de presque,tous les grands lac's qui se tronvent dans lo voisinage de la hauteur des terres.

Toute la bande occupcée par le systime laurentien forme un plateau dont le point le plus élevé, elitre les sources de la rivièro Hanmah et celles du Saint-Maurice, n'excède guire 1300 pieds de hautenr au-dessus du niveau do la mer. Eu gagnant vers l'onest, ce niveau s'abaisse graduellement : dans la région du lac Abittibi, ii n'est que de 857 picds, puis s'élève à une douzaine de cents pieds daus la vallée supérieure de la Mattagami, cutro la hauteur des terres et la PortageLong. Il n'est que de 710 pieds au nord du lac Winnipeg et il n'atteint pas 850 pieds daus touto la région situóo au uord-onest do co derifier point, e'est-à-dire dans lo grand plateau où la Churchill et la Nelson ont leurs sources.

Au nord cle cette bande do terrain laurentien, à partir de l'LastMain et en allant vers l'onest, la surface du sol ost mic, bien moins élevée et s'abaisse graduellement en gagnant la région traversée par la rivière Nelson. De 857 pieds de hauteur qu'il a daus les environs du lac Abittibi, ce nivean descend à 500 pieds environ dans la région centrale de la rivière Moose et de ses grauds aflluents, à 400 pieds au lac Oxford et à 440 pieds au lac Fendu, qui u'est qu'nue expansion des caux de la risière Nelson, vers le milien de son cours.

A part ectte pente graduelle et uniforme, la surface du sol est unie; les dykes quel'on aperçoit à de rares intervalles n'ont pas mème les dimensions d'ume colline et ne forment quo des rochers isolés, dont l'élévation n'atteint que rarement trois ou quatre cents pieds ; la partie la plus basse et la plus unie de cette immense territoire se trouve au sud de la baie James, dans la vallée de la rivière Moose, et surtont dans celle de la rivière Albany, qui est navigable jusqu'à la chute Martin, à 250 milles de son embouchure.

A l'est, daus la région de l'East-Main, les terres sont peu úlerées jusqu'à une trentaine de milles dans l'intérieur; mais plus loin elles se soulìvent considérablement et atteignent jusqu'à 2000 pieds de hauteur dans le platean central du Labrador, d'où vieunent toutes les grandes rivières qui éconlent leurs caux daus la partic orientale de la baie James et de Ja baie d'Hudson.

Dans tont lo bassin méridional de cettebaie, on ne rencontre nulle part des chaines de montagues ni des lignes de hauteurs continues. A proprement parler, les rivieres ne forment pas de bassins, 
mais coulent à travers ees grandes plaines daus des cannux qui sont au noème niveau que la surface générale du scl. Liaction érosivo des eanx de l'époque diluvienno s'est exercóe dans la région situéo au sud des Laurentides et n' $₫$ pas affecté la configuration superficielle du sol au nord do ee grand platean. Durant la période dilnvienue, le cours des immenses étenclnes d'eau qui recourrnient lo globe allait du nord au sud et, arrêtó à sa bise pin la barrière quo Iui opposait la cha1ne des Laurentides, ce courant n'a pu aroir aucun effet sur le bassin comparativement bas qui aroisine la partie méridionale de la bain d'Hudson. C'est cé qui explique pourquoi la surface do ce bassin est si rinie et si pen affettéo par l'érosion. Les seules traces d'érosior qu'on rencontre dans cette région sont le résultat de l'action des glaces. Ces stries glaciaires se rencontrent surtout dans l'East-Main, où leur direction est presque toujours entre lo snd et l'onest, ce qui correspond à pen près avec la direction générale des stries glaciaires de tout le littoral de l' $\Lambda$ tlantique, depuis la Mas. sachnsetts jusqu'an détroit d'Hudson. 'La rivière Nolson offre un des exemples les plus remarquables do ces érosions causées par lo mouvement des glaces. Fintro le lac Sipi-Wesk ct le lac Fendu, où son lit est comparativement profond, la carité qu'elle remplit a été creusée par un glacier,

Ces stries ou érosions causées par lo monvement des glaces sont tris nombreuses au nord du lac Winnipeg, dans le platean où les rivières Nelson et Churchill prennent leurs eaux. Co platean est rempli de lacs crensếs dans des terrains de formation cristalline. Ces lacs out évidemment été creusés par les glaciers à l'époque oì ces derniers ont traversé la barrière da roches solides que lezir opposait la cha1ue des Laurentides. Dans toute cette contrée, lin surface des roches atteste l'action érosive des glaces. La direction des stries est g'éneralement sud-onest et ne dévio que dans les endroits où les rocher's, plus forts que les glaciers qui les heurtaient, ont fait dévier cens derniers de leur course réguliêre.

Dans la région qui avoisine la baie James, il n'y a pos de traces d'érosion par les glaces. Durant toute la période où ces dernières ont été en mouvement, ces terrains étaient submergés et trop bas sous l'ean pour être attaqués par les glaciers qui passaient à la surface. C'est un peu ce qui explique pourquoi cette région est si unie, sans compter qu'étant d'origine archéenn̊, sa surfacen'a jamais été affectée par les bouleversements qui ont souleré les contrées plus éloignées.

Ce que nous venons de dire indique assez qu'il u'y a pas de montagnes dans le bassin méridional de la haie d'Hudson. C'est à peine si l'on pent déconvrir quelques buttes isolées qui ne s'élèvent gìère au-dessus du niveau général du sol pour mettre plus en reliefl'uniformité générale de ces grandes plaines siluriennes. Il n'y a pas même de ces petites collines, formées par des amas de gravier ou d'autres matières diluviennes, qu'on rencontre dans les plaines de la Saskatchewan, où elles ont été accumulées par les courants qui circulaient eatre la mer des Antilles et la baie d'Hudson. Ici les caux retenues 
captives par les hanteurs qui environnent la baie au sud, ne pouraient former ancuns courants, et les soules mutières diluviennes qu'on trouve a la surfice du sol sont les aggrégations glaisenses quo les glaciers laissent tomber en se fondant. après les avoir enlevés aux terrains neptuniens situés au nord do la baie d'Hudson. Ces dépóts n'ont pas ju s'uccumuler, puisqu'ils se dégagenient do glaises súdentaires, ni former les collines et les turgescences qu'ou rencontre dans les plaines de l'onest.

L'étendue de bon sol arable compris dans cette région est immense. Ce sol se compcse d'une argile yni donne dans notre province les terrains agricoles les plus fertiles. Presque partont la glaise forme it la surface des couches profondes et qui constituent nno terre arable inépuisable. Ces conches do glaise sont sonvent entremêlées de gravier, ce qui ne fait que les rendre plus avantageuses pour la culture. Cette argile est quelquefois plastique ; mais, en général, elle a été accumulée par l'action des eaux et des glaces et alors elle est plus friablo et beaucoup plus facile ì cultiver.

Ces terrains agileux s'étendent depuis le lac Mistassini jusqu'i la riviìre Churchill, dans tout le pays plat et peu élevé qui entoure la partie sud do la baie d'IIudson, c'est-à-diro qu'ils occupent une aire d'environ 250,000 milles carrés. C'est dans la région égontéo par la rivière Moose et la rivière Albany qu'ils ont le plus grand développement et qu'ils paraissent former le meilleur sol. Dans nue étendue nussi vaste, on conçoit qu'il doit y avoir des exceptions, des espaces rocheux ou sablomeux; mais ces exceptions ne font que confirmer la règle générale et l'on pent dire sans crainte qu'il y a dans cette plaine silurienne des millions et des millions d'acres de bonne terre arable. C'est à pen près l'opinion du Dr Iell, qui a pendant plusieurs années parcourn tout ce pays en sa qualité de membre de la Commission géologique du Canada. Voici cequ'il dit dans lo rapport sur une exploration faite en 1875 :

"Entre les grands lacs et la baie James, la nature des terrains diffëre considérablement dans chacune des denx formations géologiques qui so partagent cette région, c'est-ii-dire le plateau laurentien et huronien, puis le bassin paléozoïque et probablement tertiaire de la baie James. Le premier est un pen ólevé, d'uni surface ondoyante et parsemé d'un grand nombre de lacs, au lieu r ae l'autre est bas uni, marécageux et, autant qu'ou a pu lo coustater, ne renferme généralement pas de lacs, formant au point de vue géographique comme an point de vue de la géologie un bassin bien distinct, limité visiblement par une bande de roches anciennes sur les cinq-sixièmes de sa circonférence.

"Quoique le platenu laurentien et huronien situé entre les grands lacs et la baie Jannes puisse être désignó comme un pays rocheux; je crois cependant que la partie de cette aire dans laquelle les roches nues sont exposées est bien moins g'tande qu'on ne le suppose généralement. J'en suis arrivé à cette opinion après avoir examiné ce platean à des centaines de places, $i$ distance des bords des lacs et des 
rivières, sur une étendno de deux mille milles carrés, entrè la rivière Ontaonais et lo lac Winnipeg. Les points rochenx et élevís sont naturollement plus en évidence, en proportion de lenr étendue horizontale, que les autres parties du pays, en riême temps que les portages, qui sont it peu près les senles parties vues par les voyugeurs ordinaires, sont presque toujours dans les endroits les plus rocheux des vallées et des terraius bas. Ces circonstances rénnies donneit aux visitenrs d'occasion une plus mauraise opinion do ces régions qu'elles ne méritent. Des débris d'une espếce quelconque reconvrent réellement la plus grande partie de cette aire, et sur une très grinde étendne lo sol est plus ou moins propre à l'agricnlture. J'en ni décrit la nature d'une manière précise, pour différentes régions, daus les rapports que j'ai faits depuis 1869 jusqu'ì ce jour. Relativement à cette espèco de prys, il est constaté par l'expérience, dans le district d'Algoma et ailleurs, que la ln quantité de terres enltivables, lorsque les établissements se forment, est tonjours plus grande qu'elle ne paraissait l'étre lorsque tout était dans l'état naturel. D'une manière générale, il y a pent-être une plus grande proportion de hon sol dans le platean qui se tronve au nord que dans celui qui se tronve au sud de la hauteur des terres. Quant il la région comprise dans le bassin paléozoïque de la baie James, la trop grande muiformité de sa surface est plutôt un désarantago qu'un avantage, car, quoique lo terrain puisse étre généralement assez élevé au-dessus do la rivièro la plus rapprochée, il paratt êtro généralement marécagenx; excepté dans la lisière qui borde inmédiatement la rivière. "

M. le Dr Bell ne parle pour ainst dire que du platean laurentien, où il constate qu'il y a beancoup de terriain cultivalble, et il exclut du sol susceptible de culture tonte la région plus basso que ce plateau. Après une exploration plus soignée et plus étendne, il est revenu sur cetto opinion, ainsi qque le constate l'extrait suivant do son rapport pour $1877-78$ :

"Dans mon rapport pour 1875, j'ai domné un aperçu général du sol et de la région située entre les grands lacs et la baie James. Fin suivant la route des cannts entre Michipicoten et lo fort Moose, le pays est plus ou moins rocheux jusqu'au lac Missinaibi. Cependant, même dans cette région, la proportion de la surface rochense ì l'aire totale semble étre comparativement petite. Mais après avoir passó les "terrains marécageux," au nord du lac Missinaibi, le voyageur ne pent pas s'empècher d'être frappé par l'alondance et la fertilité gónérale du sol exposé sur les bords de la rivière Missinaibi et de la rivière Moose, jusqu'au fort Moose. Ce sol se con pose en grande partie de terre grasse gräreleuse, de conleur bruntre, reposant sur du tuf' (till), et quelquefois sur des strates de glaise on de roc solide, roc qu'on aperçoit rarement, exceptó anx principaux rapides et aux chutes. Mais dans le tiers central de la section comprise entre le lac Supérieur et la baie Janes, on depuis la rivière Brunswick jusqu'an Portage-Long, la surface du sol est généralement formée par une glaise de couleur légère. A plusicurs endroits, j'ai examiné le terrain 
jusqu'it une distance d'un mille ou deux de la rivière, pour m'assurer de la nature du sol, et je l'ai trouvé excellent partout, mais tendant ¿ derenir plus marécagenx à mesure qu'on s'óloigne de la rivière, daus la rígion devonienno qui so trouvo plus bas que le Portage Jongr. Lin traversant une anssi grande étenduo de forêt presque continue, ou est porle a oublier la valeur qu'une aussi vaste région peut avoir pour les fins agricoles; mais l'exemple des formes qu'on exploito au fort New-Brunswick et an fort Moose montre, sur une petite échelle, ce que l'on pourrait fuire dans une grande partio de ce pays. .Te n'ai pas de dunle qu'un jour a venir ce lerritoire fera virre une nombreuse popmlatron."

Ce qui précèdo pent donner une juste idée des terrains qui entonrent la baic James et s'étendent an nord du platenu laurentien depuis le lac Mistassini jusqu'aux sources do ln rivière Albany. Dans les environs du lac Mistassini, le sol est également bon, insi quo le fait est constaté dans le rapport de M. James Richardson, dont l'expérience et la compétence ne sauraient être révoquées en doute. "Le terrain se compose d'uno terre grasse sablonnense, bien propre anx fins agricoles." Cette contrée repose sur des strates de caleaire dont la décomposition produit un sol fertile. I la surface des terrains est unic, ne s'élerant pas plus de trente pieds au-dessus du lac, ce qui rend cette région favorable à l'agriculture. " 1

Un sol aussi bon, sinon meilleur, convre la région dans laquelle so trouvent lo lac et la rivière Abittibi. "I.o lac $\Lambda$ bittibi est environné do tous les côtés par une terre glaise unic. En plusieurs endroits, cependant, le ros perce la surface de la grlaise. Ceci so voit partictilièrement le long da côté sud do la partie supérieuro du lac, où des collines dioritiques approchent de ses bords; mais mêmo dans ces endroits, il y a génér alement uno lisière de terre glaise le long dn rirage. An nord, et surtont au nord-ouest. lo niveau de la terro glaise semble presque continu et il est bien connu que ce sol se prolonge dins celle divection jusqu'aux rivagres de la baie d'Hudson. Plusieurs acres de ce sol de glaise sont cuitivés an poste de la compagnie de la baie d'Hudson, un lac Abittibi, et avec des résultats satisfaisants." 2

An nord-ouest des sources de la rivièro Albany, le terrain semble itre preschu'aussi bon, nussi propre à l'agriculture, dans la région du lac de l'Ile, du lae Oxford et du lac Genou, anx sources de la rivière Inyes. "De grandes étendues de terrain bas et sablomnenx se rencontrent aux environs des lacs Oxford et du Genou, particulièrement du côté nord. Les endroits les plus élevés, quoiqu'ils ne sont pas rocheux, sont ordinairement recouverts par une glaise forte, de couleur légère, et un sol semblable, avec plus ou moins de terroir, se

1 Rapport Géolsyique, 18'70-72, pages 302 et 303.

2 Rupport Giologigute, 1872-73-3[COuat, page 134. 
rencontre daus la valléo de la rivière à la Truite. Le fort Oxford est bâti sur un sol do glaise forte, dans lequel l'orge et toutes les sortes do légnmes de jardin croissent à perfection. Au nord du lac, vis-à-vis le fort Oxford, le terrain s'élève a environ 200 pieds de hanteur et parait être plus élevé que dans aucun antre endroit de cette région. Sur la route conduisant de la baie Jackson, à l'extrémité orientale du lac Oxford, à la partie supérieur du lac Dien, le pays est plus accidenté que dans le voisinage du lac Oxford. Qnoique la surface soit en général moins unie, M. Cochrau estime que la proportion des rorhes aux autres matières formant la surface du sol ne forme gu'un quart de'la superficie totale. Le sol, on les débris superficiels, se compose de terroir, de gláise, de snblo et de gravier, ou d'un mélange de toutes ces matières. Autour du lac des Iles, en s'éloignant un peu des bords du lac, on troure généralement mn terrain recouvert par un bon sol. A près aroir exploré les environs de se lae, M. Cochran a inscrit dans son livre de notes les remarques suirantes: "Lo sol que j'ai ru en faisant le tour du lac est réellement très bon, se composant généralement a'une gglaise brune légère, mêlée en beancoup de places avec nn pen de gravier fin. Dans chaque cas, lorsqu'on s'éloigne ì une certaino distance, lo roe qu'on voit au bord du lnc disparait ou est reconvert de terre, puis les arbres sont plus gros et d'une plus belle venue que sur le bord dc l'eau. Il y a un très bon jardin à ce poste et je n'ai certainement jannis ru de pommes de terrearoir meilleur apparence que celles qu'il y a ici." 1

L'exactitnde de ces renseignements si farorables, sur la nature dn sol de la régivin où la rivière Ifayes a son conrs supérieur, sont confirmés par le Dr Bell, qui arait exploré ce pays l'nnnée précédente. " La nature des dépôts superficiels, dit-il, et du sol, a été exposée en détail dans la description des régions explorées durant cette saison. La prédominance, dans une région aussi étendue, d'une glaise de conleur légère, formant souvent un bon sol, exempt de cailloux, est ru fait qui a beaucoup d'importance relativement à la raleur future de cette partie du pays. On dit que ces dépôts recouvrent la plas rrande partie de la région située entre la rivière Nelson et la rivièo Ćhurchill et même avdelà... Dans cette région, le roe massif est gónéralement recouvert par la glaise grise qui domine partout et qui, en certains endroits, est cuite par le soleil et se crerasse, mais dans d'antres, forme un sol meuble, friable et d'excellente quaiité. Comme de raison, beancocp de roc vif apparait sur le bord de l'eau, le long des principales rivières; mais, méme là, la partie supérieure dess berges, y compris celle des petites íles, se compose généralement de glaise." "2"

"Le caractère général de la contrée qui bordo la rivière Nelson de-

1 Raphort Géoloyique, 1878-79, pages 32, 33c.

2 Rayporl Géologique 1877-78, pages 38 et $24 \mathrm{cc}$, 
puis lo fort Norway jusqu'a la rivière Goose-IIunting est joliment nniforme. Toute la surfice clu terrain parait ètro recouverte d'une glaise grisître, qui formo un excellent sol. On aperçoit les roches sous la glaise dians les fles et sur les bords de la rivière et clans le roisinago des rapides elles surgissent sur une plus on moins grande étendue. Intre le lac Sipi-Wesk et le lac Fendu, où la rivière coule dans in seul chenal, creux, droit et d'une largent presqu'uniforme, la berge orientale se compose de glaise, presque continuellement, tandis que la borge gauche laisso roir plus on moins de roches.

"Lo beau sol do glaiss que l'on rencontre dans la moitié supérienre do la vallée de la rivière Nelson a déjà été mentionnó. Tous les rap. ports s'accordent pour constater qu'un sol de glaise également bon, avec de rares parties rocheuses, s'étend de là vers le nord-ouest à travers la régrion égonttée par la ririère du Bois-Brûlé et jusqu'à la rivière Churchill.

" On peut dire qu'un terrain semblable s'étend tont lo long de la ronte des bâteaux entre le fort Norway et le fort York. Dans la moitié nord de cet te distance (au delì de 300 milles) la surface dn pays, excepté dans lo roisinago des rivières, parait être généralement unie, humide, courerte de couches monsseuses et supportant une forêt composée de petites épinettes blanches et rouges. lin regardant du sommet de la colline Brassy, près de la rivière Hill, la surfice du pays parait être généralement unie dins toutes les directions; mais on voit anssi de petites ondulatious et des buttes de terre entremèlées do lács. On dit que cetto vue doune en moyenne tine bonne idée de toute la région comprise entre le platean laurentien et lo. mer, depuis la rivière Wanisick jusqu'à la rivière Churchill, ce qui forme une étendue de peut-être cent mille milles carrés. On clit qu'une région de bons terrinins forme une aire considérable au sud de cette ronte des bîteanx, y compris ies terres qui enviromnent le lac Dien et les sources de la rivière Severn." 1

Au nord-ouest du lac Winnipeg, sans être d'me occurrence aussi générale que dans les régions que nous venons de décrire, le bon sol irable est loin de faire défaut . La région comprise entre le lac Travers et lo lac Moose, qui se troure a quatre-ringts milles à l'ouest, a été partielloment explorée par M. Cochran, de la Commission géologique, en 1879 . D'après son rapport, lo lac Hill, qui se trouve vers le millieu de"la région explorée, " est entouré par des falaises de belle glaise stratifiée, qui ont de vingt ì vingt-eing pieds de hanteur. Le calcaire fossilifêre se rencontre à l'extremmité sud-onest de ce lac "... Du calcaire semblable à celui du lac Winnipeg a été r’n en qualques endroits sur la rire nord-ouest du lac Moose. "Le lav des Pieds-Noirs se truuve plus loin rers le nord-ouest. "Les bords immédiats ont en ri-

1 Dr Bell-Rapport du ministre de l'Intérieur pour 1878, partic III, pages 7 et 0.

2 Dr Bell-Rupport du ministre lle l'Intírieur pour 1878, partie III, pages 7 et 3. 
ron dix pieds de hanteur ; mais au sud, la berge a do cinquante à soixante pieds d'élévation et paratt être formée par nu sol de glaises supportant uno forte forêt d'épinettes. Les mémes calcaires se roient en un grand nombre d'endroits, mais on n'a pu y déconvrir ancuns fossiles.

Ces données, si maigres qu'elles soient, établissent elairement que dans cette région, comme partout ailleurs, le sol calcaire et la glaise ne manquent pas.

Le plateau dans leqnel la rivièro Churchill a ses nombreuses sources renferme des terrains arables bien supérieurs à cenx de la région que nous verrons décrire. La partie compriso entre le portage Methy et le lac Vert, distance de plns de deux cents milles, a été exploréo par le professeur Macoun en 1875. Nous extrayons de son rapport les notes qui suivent.

"La distance entre le Portage-la-Loche et l'Ile-ì-la-Crosse est estimée à 130 milles. Entre le portage et le lac du Bouf, le pays n'est guère antre chose qu'une tourbière on un marais ; mais du moment qu'on atteint l'extrémité sud du lac, il se produit un changement marquant et la forêt antour du lac Clair (ou lac des (Enfs) so compose presque toute de tremble... La rivière Crense et le lac Lacrosse sont tous les denx entonrés de forêts de tremble, forêts qui dans le nord indiquent toujours un bon sol. Le fort (de i'sle ì la Crosse) est agréablement situé sur un bras da lac... Le sol, au fort, est jauvre, comparé à celui de la rivière ì la Paix. Il se compose surtout de terro janue mêlée à beancoup de sable blanc. En s'éloignant du lac, le sol s'améliore et se compose en grande partie, d'une terre glaiseuse... Nous suivimes la brazche ganche (entre le lac de l'tle à lia Crosse et le lac Clair) et remarquâmes de suite une amélioration dans l'apparence du pays. De beaux bosquets de trembles et de peupliers bordent la rivière sur les deux côtés et tont le pays paraft être convert par les mémes bois. Toute la régrion ruog cette après-midi est bien propre pour faire des établissements, et des myriades de poissous fourmillent dans la rivière. Nons partimes an premier petit jour, et après avoir passé deux rapides, le courmnt cessa et les bords de la rivière étaient recouverts jusqu'à l'ean par dessaules, des annes, des cornonillers et des peupliers. Le sol parait être d'excellente qualité. et où il n'y a pas de hois, il est couvert de vesces, ete. Li rivière se bifurque encore. La branche gauche conle à travers mu bean terrain. Les bords sont formés d'alluvions et ils n'ont que dix jieds de hautenr. Le sol est tris riche des deux côtés.

Le lac Vert, vn de la décharge de la rivière, est une belle nappe d'ean, d'environ dix-hnit milles de longeur dn nord an sud, arec une largenr moyenno d'un feu plus d'un mille. De chaque côté le terrain s'élère graduellement, à partir des bords de l'ean, qui sont formés par des galets, et il est couvert d'une épaisse forêt de grands trembles et penpliers, ce qui indique un sol bon et trés productif. Le sol dans lo voisinage du lac Vert est d'excellente qualité. Cette région' est propre 
à la colonisation d'un bout a l'autre, lo sol étant d'excellente qualité et tout a fait libre d'humidité."

Disons de suite que le climat est aussi bon que le sol et que dans toute cette région, on cultive avec succès toutes les céréales, mème le blé.

Au sud-juest, une autre branche du fleuve Churchill, la rivière aux Castors, traverse une grande valiée dont le sol est excellent et très propre à l'agriculture sous tous les rapports. Ce fait est constaté par tous ceux qui ont visité cette localité. "La rivière aux Castors, dit Mgr Taché, I est navigable pour des cauots d'écorce. Si l'eau pouvait se maintenir a cette hauteur, cette rivière serait d'autant plus avantageux qu'elle traverse un pays en grande partio propre à la colonisation.

Cette appréciation est plus que corroborée par le chef facteur Ar. chibald McDonald, daus ses notes sur le Voyage en Canot de Sir John Simpson. "La partie supérieure, dit-il, ou la rivière aux Castors, de cette ligne de partage des eaux de la rivière Churchill, intermediaire et, de fait, lors de la crue des eaux, se déversant des derx cótés, entre le bassin de la Saskatchewau et celui du Mackenzie, est un beau pays pour faire des établissements. Le sol est bon et fort, bien boisé, méme fortement boisé de pins magnifiques et d'autres bois précieux, et ce territoire se troure dans la zone-" la zone fertile "-dont nos écrivains parlent dans les livres bleus. C'est vrai, mais j'espère leur démontrer avant longtemps, qu'en décrivant les limites de leur " zone fertile "ils ne vont pas assez an nord et à l'ouest. "2

Enfin du côté de l'est, l'East-Main, qu'oul regarde ordinairement comme une région inculte, renferme aussi une certaine ótendue de trrre arable et eultivable, au témoignage du Dr Bell. "Lo long du rirage est de la baie James, depuis le voisinage du fort Rupert jusqu'au car Jones (distance de plus de 300 milles), il y a une bande de terrain, dont la largeur moyenne varie de vingt à trente milles à partir du rivage de la mer, qui, d'uprès tout ce que j'ai pu apprendre des autres et constater par moi-même, pourrait apparemment avoir un jour une certaine rale ur agricole. Vue de la baie, elle parait avoir une surface verdoyante et elle incline graduellement jusqu'au rivage. Ce terrain est boisé d'épinettes, de tamaracs, de peuliers et de petits bouleaux. Au fort George, j'ai vu une quantité be bons billots d'épinette qui avaient été descendus sur la Grande Rivière pour servir à la construction. Beaucoup de ces billots mesuraient deux pieds de diamétres au bout... Le sol de cette bande de terrain est généralement sablonneux, :eposant sourent sur des strates de glaise grisâtre qui sortent parfois à la surface, au-dessus desquelles se trouvent des cail-

\section{Esquises sur le Nord-Ouest, page 40}

2 Peace River, a Canoe voyagefrom IIudson's Bay to Pacifc, by the sate Sir George Simpson, Pags 56. 
loux ou des roches solides; mais la surface se compose de sable ou de glaise. Les jardins du fort Rupert, de l'East-Main et du fort Goorge prouvent que les pommes de terres et tous les légumes ordinaires poussent bien dans ce sol." "

En résumant toutes ces données. on arrive n'écessairement à la conclusion que le grand bassin méridional de la baie d'Hndson renferme une immense étendue do bon sol arable. On a généralement une opinion contraire, opinion qui est malheureusement aussi fausse qu'elle est en vogue; mais, enfin, les faits contredisent positivement cette opinion, et il faut bien se rendre à leur évidence. La moitié au moins, en mettant les choses au pire, de l'étendue de ce grand bassin renferme nn sol susceptible de culture et généralement d'excellente qualité. Ces terrains argileux sont identiques à ceux de la presqu'ile de la province d'Ontario et de la plaine comprise entre le Saint-Laurent et le Richelien, dans la province de Québec, deux des régions úgricoles les plus fertiles et les plus riches du Canada. Aux environs de la baie d'Hudson, comme dans les deux autres localités que nous avons mentionnées, les terrains appartiennent aux différents étages dé l'âge silurien et au nord comme au sud des Laurentides, on rencontre un sol de même nature et de même fertilité, également capable de rétribuer au centuple la population qui voudra bien se donner le trouble de le mettre en bon état de culture. Le seul désavantage que présente ce terrain, c'est l'uniformité de sa surface," ce qui rend l'égouttage plus difficile, mais ce désavantage n'est pas plus grand au nord qu'au sud des Laurentides et la main laborieuse du cultivateur réussira aussi facilement à assécher les terrains si riches de la baie James qu'elle a réussi à assécher ceux de la vallée du Saint-Laurent. Quand le sol est bon, on trouve aisément moyen de le débarrasser de ce qui peut l'empècher de produire tout ce qu'il est susceptile de rapporter.

En estimant, comme nor l'avons fait, que le sol arable forme au moins la moitié du bassin sud de la baie d'Hudson, nous arons une étendue de 250,000 milles ou 160,000,000 d'acres carrés. Nous verrons plus loin qu'd raison de la rigueur du climat, ce; terrain cultivable n'est pas tout susceptible d'exploitation ; mais, quant à la nature du sol exclusivement, c'est là l'aire des terrains arables. C'est-a-dire quo dans cette région, l'étendue du terrain arrable excède d'une cinquantaine de mille carrés l'étendue totale de la province de Québec. Et nous mettons le tout au plus bas, car la partie que nous retranchous est bien supérieure, comme sol arable, à la plus grande étendue des terrains que l'on cultivo avec succès en Norvège en Suède, ainsi que dans la Finlande, pays situés dans la même latitude et soumis aux mèmes influentes climatériques que le bassin méridional de la baie d'Hudson. A elles seules, la Suède et la Norrège ont une population de 6,000,000 d'habitantis : si notre région du nord se peuple dans la

- Rapport Glologique, 1877-78, pages 24-25 
proportion, il est facile de prévoir l'avenir qui eat réservé a cette partie du Calnada.

\author{
CHAPITRE IV \\ CLIMAT-T'LMPÉRATURE-COMPARAISONS AVEC D'AUTRES LOCALITKS \\ SAISONS-OPERATIONS AGRICOLES
}

- Cetto partie de notre étude est la plus importante. Pour déterminer si un pays est habitable ou ne l'est pas, il faut d'abord constater si la température est assez élevée pour permettro à la végétation do prendre tout le développement dont elle est susceptible, si les produits de l'agriculture penvent arriver à leur pleine maturité. Quand la question du climat est résolue, toutes les autres questions relatives à une région comme centre d'occupation et d'établissement sont réso. lues, car dans les climats habitables, le sol est toujours cultivable. Les montagnes, la configuration physique et les caractères géolo. giques du sol peuvent affecter sa fertilité ; mais il est bien rare que dans une région jouissant d'un climat propice aux opérations agricoles, ces circonstances puissent reudre le sol absolument impropre à la culture. Les régions stériles constituent l'exception et non pas la règle générale.

L'idée principale de la climatalogie des latitudes tempérées de l'Europe et de l'Amérique, c'est la correspondance ou la similitude du climat dans les latitudes et les positions géographiques semblables. Ceci comporte, pour ainsi dire, une similitude d'arrangement dans les climats, qui aide beancoup à déterminer les conditions qui les ré. gissent. Ainsi l'tle de Vancouver occupe une position analogue à celle - des Iles Britaniques-elle se trouve dans la mème latitude et du même côté du continent et par les obserrations qui ont été faites dans cette 1le, nous constatons qu'il y a dans l'ile de Vancouver les étés frais, les hirers chauds et l'humidité générale de l'admosphère qui caractérisent le climat des Iles Britaniques. En dehors de ce fait, il faut bien pen d'observation pour arriver à la conclusion que l'adaptation à l'agrieulture et la capacité productive de l'Augleterre doivent se retrouver au mène degré dans l'tlo de Vancouver, ainsi que l'ii: mense systeme d'opérations industrielles et commerciales qui accompagnent toujours l'agriculture et constituent ce qu'on appelle un pays habité et civilisé.

Pour les autres régions de l'Amérique, et particulièrement pour celles de l'intérieur; comprises entre $47^{\circ}$ et $60^{\circ}$ de latitude, on peut

- Blodget, Climatology of the United States. 
tirer les mèmes analogies des climat européens. La Norvège, la Suède, la Russie centrale et la partie nord de l'Allemagne oecupent; au point de vue climatérique et géographique, uno position semblable à celle de la partie sud du bassin de la baie d'Hudson, et par conséquent, celle-ci doit ètro également susceptible de culture, également capable de supporter une nombreuse population dans l'aisance et le confort:Actuellement, cette région est inhabitée Cela ne dépend pas du climat, mais de l'iguorance qui existe au sujet du elimat. On est généralement sous l'impression que tout le pays qui entoure la baie James et la partie sud de la baie d'Hudson n'est qu'un pays de glace, à peine capable d'être habité par des Esquimaux; on suppose que l'intérieur a le méme climat que le Labrador, à l'est, et les régions arides situées au nord du détroit d'Hudson. Ces régions glaciales sont à peu près les seules dont le climat ait été étudié par les explorateurs qui ont voulu se rendie au pôle nord, et quand ces explorateurs nous disent, dans leurs récits, que le mercure descend jusqu'à cinquante degrés au-dessous de zéro dans les régions qu'ils ont risisitées, que la glace ne se fond pas, même en été, dans les mers où ils ont uavigué, on est porté à crrire que les mêmes froids se font sentir jusque dans la partie méridionale de la baie d'Hudson. Pour beaucoup de gens, les mers polaires et la baie d'Hudson ne sont qu'une seule et méme chose. Il y a biell douze ou qninze cents milles de différence entre la latitude de la région que nous étudions et celles des localités visitées par les navigateurs qui cnt essayé de pénétrer jusqu'au pôle nord ; mais peu de gens se domnent le trouble de faire cette réflexion et l'on continue à condamner tout le bassin de notre grande méditerranée du nord comme une région glaciale et inhabitable.

Pourtant, en jettant un coup d'œil sur la carte de l'Europe, il est bien facile de voir qu'une région absolument semblable sous 'e rapport climatérique est peuplée par plus de $25,000,000$ d'habitants. Cette région forme le contour de la mer Baltique. Il y a une ressemblance frappante, au point de vue géographique et climatérique, entre la Baltique et la baie d'Hudson. Ces deux mers intérieures sont séparées de l'Attantique par des pénisules èlevées et rocheuses au centre, puis touchant au nord les eaux de la mer polaire: en Europe, c'est la Scandinavie qui sépare la Baltique de l'Atlantique, et en Amérique, e'est le Labrador. La Baltique communique avec l'Atlantique pir le Cattegat et la mer du Nord; la baie d'Hudson communique arec le même océan par le détroit d'Hudson et le bras de mer qui sépare le Groënland du Labrador.

Dans l'un et l'autre cas, la direction et l'influence des courants océaniques sont identiques. Repoussé vers l'ouest sur les côtes de l'Ecosise, le gulf-stream passe en dehors de la mer du Nord et bien loin de la Baltique, qui subit un peu l'influence du courant venant des mers froides du pôle. Le même phénomène se produit en Amérique. Repoussé par les côtes de Terreboune, le gulf-stream ne fait qu'effleurer le bras de mer dans lequel aboutit le détroit d'Hudsou, 
et c'est surtout le courant renant des mers polarres qui se fait sentir dans notre graude mer du nord.

Relativement à l'influence que les courants océmiques peuvent erercer sur le climat, le bassin de la baie d'Hudson se trouve donc dans la rnême position que celui de la Baltique.

Mais il est une autre cause qui influe sur la température, c'est la hauteur du sol au-dessus du niveau de la mer. Or, sons ce rapport, les deux bassins se trourent absolument dans la même position. Vers l'intérieur, la plaine qui avoisine la Baltique est plate, unie. peu élevée et contournée par les monts Valdai, dont la hauteur moyenne n'ercède pas onze cents pieds. Du côté de l'océan, la péninsule scandinave, avec son relief accidenté, surmonté de quelques sommets, tels que le Galdköppigue, haut de plus de 7000 pieds, escarpé du côté de l'Atlantique et s'abaissant graduellement vers la Baltique, offre plusieurs points de ressemblance avec le Labrador. Cette péninsule sépare le bassin de la baie d'Hudson de l'Atlantique. Vers le milieu elle a environ 2000 pieds de hauteur. Elle est escarpée du côté de l'acéan et elle descend en pente douce vers la baie d'Hudson. Enfin les hauteurs des Laurentides, qui couronnent la plaine unie, peu élevée, avoisinant notre grande méditérannée canadienne, occupent à peu près la mêmo position et ont la même altitude que les monts Valdai,, qui séparent la Russie centrale de la région méridionale, absolument comme les Laurentides séparent le bassin de la baie d'Hudson de la partie sud du Canada. En ce qui regarde les reliefs et la hauteur du sol, les deux bassins sont absolument semblables.

Pour ce qui regarde la position géographique, l'influence que les courants océaniques peuvent excercer sur le climat, l'élévation du sol au-dessus du niveau de la mer, ses réliefs et sa configuration, enfin toutes les causes qui peuvent affecter le climat et la température, la ressemblance, l'identité sont donc partaites entro le bussin de la Baltique et celui de la baie d'Hudson, et pour que le climat de co dernier ne fût pas aussi favorable, aussi propice que celui de l'autre, à l'agriculture et aux différentes opérations qui constituent la vie industrielle des peuples civilisés, il fandrait que la nature eût changé ses lois par simple caprice et traité l'Amérique en marâtre.

Heureusement que les faits et l'observation sont lì pour établir qu'il n'en est pas ainsi.Le climat du bassin méridional de la baie d'Hudson n'a jamais été soumis à une étude méthodique et bien coordonnée'; mais les observations thermométriques qui ont été faites en plusieurs endroits, les nctes des voyageurs, les témoignages entendus dans deux enquêtes parlementaires et les rapports si précieux des membres de la commission Géologique, qui ont exploré cette région, fournissent des éléments qui permettent de se former une bonne idée du climat de la baie d'Hudson, dans sa partie sud, et de le comparer à celui des autres pays. En réunissant toutes ces données, nous avons pu former le tableau suirant : 

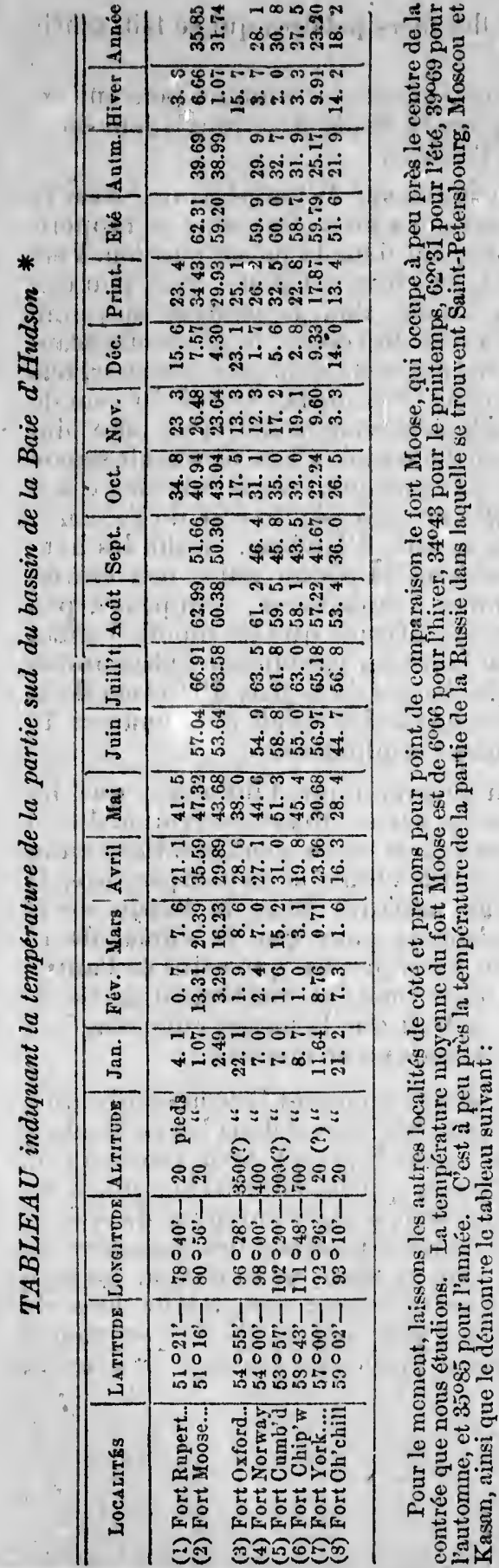

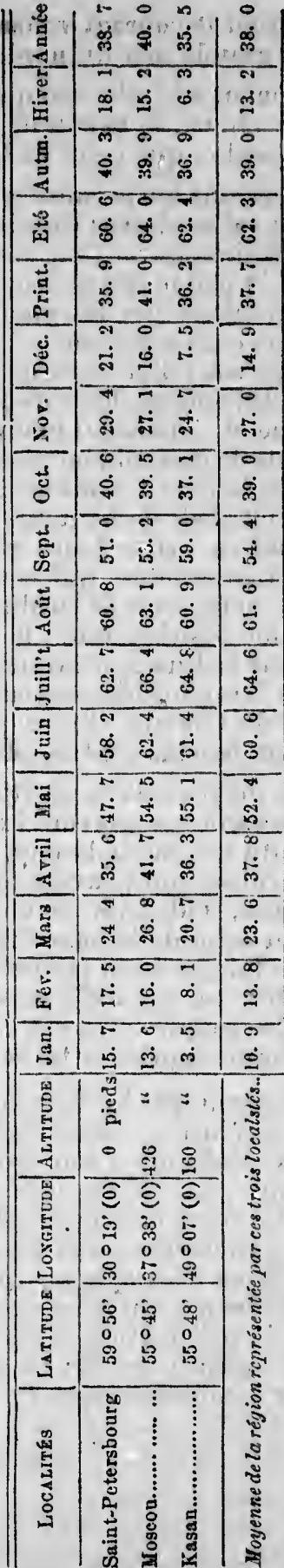

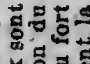

년중

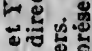

造造

웅.

总

क्ष.

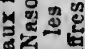

g서

En

造的

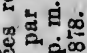

造

政屯

ํㅕㅇำ

跑

मิं

स.

प艹 की

可势

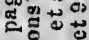

सै स्से है

हo혀

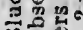

g.

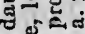

象跑

글

동현.

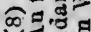

ऽ

Q⿹

कि

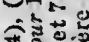

$\checkmark$ :

क्षे

-

욜

ठำ 조

ํํㅇ

घ.

s. है.

농

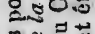

.

동 记

$\checkmark$.

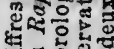

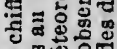

m

글

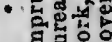


Comme il est facile de le coustater eu comparant ces tableaux, la température du fort Mooso est à peu près exactement la mème que cello de Saint-Pétersbontrg, la ville la plus populense et la plus avancée de la Russie, dout elle est la capitale. L'hiver est $11^{\circ} 44$ plus froid au fort Moos? qu'à Saint-Petersbourg; mais, en revanche, l'été est $1^{\circ} 71$ plus chaud. La températ nure do l'automne ne diffère que de $0^{\circ} 61$, et celle du printemps de 0 47. Eufin la température anuuelle de la capitale de la Russie n'est quo de $2{ }^{\circ} 85$ plus élerée que cello du fort Moose, ce qui s'explique par la différence qu'amènent les températures hibernales. Il serait difficile d'établir plus clairement, d'une manière plus irréfutajle, qu'au point vue du climat l'immeuse territoire dont lo fort Moose forme lo point central est parfaitement habitable. Quand les czars de Russic font leurs lélices ì habiter ce climat, il semble qu'il serait plus qu'absurde de soutenir qu'il est trop rigourenx pour permettre d'y former des établissement prospires,

Les chiffres représentant la moyenne des trois villes russes accusent une différence de $2^{\circ} 27$ pour lo printemps, de $6^{\circ} 54$ pour l'hiver et de $2^{\circ} 15$ pour l'année; mais il faut bien remarquer qu'ils s'appliqueut a une région fort étendue, couvrant un degré do latitude et quatre de longitude, dont la plus grande partie se troure vers le sud, dans les provinces de Mocnny et Kazan. Pour faire uno comparaison équitable, il faudrait faire entrer en ligne de compte l'immense région qui s'étend à plus de deux cents milles au sud du fort Moose, et alors la différence que nous renons de constater en favenr des provinces russes ferait plus;que disparaitre. Du reste, malgré ce désavantage, l'crcès des températures de l'été ct de l'automne est d'une fraction en faveur du fort Moose, ce qui fait plus quo balancer l'autre excès pour les deux saisons qui ont bien moins d'importance an point de vue agricole que l'hirer et le printemps.

Comparé à cếlui de la Suède, le climat de la région du fort Moose est plus élevé en été, à peu près égal en printemps et en automne, plus froid en hiver et de $3 \circ 76$ moins élevé pour toute l'année. Cette comparaison ressort clairement du tableau suivant : 


\begin{tabular}{|c|c|c|c|c|c|c|}
\hline ' Loealites & Latitude & Prinlempn & Ete & Automns & Hiver & Annfe \\
\hline Fort Moose $\ldots \ldots \ldots \ldots \ldots \ldots \ldots \ldots$ & $6101 \mathrm{R}$ & $34^{\circ} 43$ & $62 \circ 31$ & $39 \circ 60$ & $6^{\circ} 60$ & $35 \circ 85$ \\
\hline Sudde $: \ldots . . . \ldots \ldots \ldots \ldots \ldots \ldots \ldots \ldots$ & & & & & & I \\
\hline Lillnd .......... & $65 \circ 42$ & $41 \circ 78$ & $62{ }^{\circ} 66$ & $47=69$ & $29 \circ 87$ & $45 \circ 10$ \\
\hline 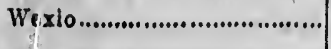 & $56 \circ 53$ & $41 \circ 53$ & $63 \circ 45$ & $14 \circ 40$ & $28 \circ 23$ & $44 \circ 56$ \\
\hline Gottenburg........................... & 07011 & $43 \circ 33$ & $62 \circ 13$ & $47 \circ 77$ & $31 \circ 51$ & $46 \circ 34$ \\
\hline 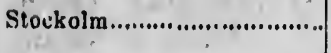 & $59 \circ_{21}$ & $38 \circ 20$ & $60 \circ 40$ & 44040 & 26000 & $42 \circ 30$ \\
\hline Gurlstad $\ldots \ldots \ldots \ldots \ldots \ldots \ldots \ldots \ldots$ & $59 \circ 23$ & $40 \circ 40$ & $61 \circ 19$ & $44 \circ 43$ & $27 \circ 10$ & $43^{\circ} 28$ \\
\hline Faluh ............................... & $60 \div 39$ & $37 \circ 82$ & $57 \odot 90$ & $11 \circ 51$ & $18^{\circ} 71$ & $39 \circ 02$ \\
\hline Hernosund $\ldots . . . . . . . . . . . . . . . . . . . . .$. & $62 \circ 28$ & $32 \circ 00$ & $56 \circ 06$ & $388^{\circ} 41$ & $17^{\circ} 35$ & $36 \circ 36$ \\
\hline Ostersund $\ldots . . . . . . . \ldots \ldots \ldots \ldots \ldots$ & $63 \circ^{\circ} 24$ & $34^{\circ} 03$ & $56 \circ 11$ & $31 \circ 57$ & $17 \circ 10$ & $35 \circ 80$ \\
\hline Umen.................................. & $63 \circ 50$ & $33^{\circ} 14$ & $57^{\circ}+48$ & $37 \circ 67$ & $13 \circ 07$ & $35 \circ 42$ \\
\hline Knontokis .......................... & $68 \circ 50$ & $24^{\circ} 96$ & $54 \circ 61$ & $27 \circ 07$ & $10 \circ 36$ & $27 \circ 04$ \\
\hline Moyenne de ces dix localitts... & (............. & $35 \circ 72$ & $59 \circ 21$ & $40 \bigcirc 83$ & $21 \circ 03$ & $39^{\circ} 61$ \\
\hline Fort Moose.......................... & ............... & $34^{\circ} 43$ & $62 \circ 31$ & $39=69$ & $6 ? 60$ & $35 \circ 85$ \\
\hline ifförence $\ldots \ldots . . . . . . . . . . . . . . . . .$. & , o... & $-1 \circ 39$ & $+3 \circ 10$ & $-1 \circ 23$ & $-14^{\circ} 37$ & -3076 \\
\hline
\end{tabular}

Les chiffres représentant la température de la Snède sont le résultat d'une série d'observations cou rrant une période de plus de dixans et compilés avec le plus grand soin par le colonel Forsell, de Stockolm. Les dix localités auxquelles ces chiffres s'appliquent sont disséminées dans toutes les parties de la Suède, en sorte qu'ils donneut mue idée fort exacte du climat de tont le pays. Il n'y a que denx localités dont la température d'été excède un peu celle du fort Moose ; ce sont Lund et Wexio. Celle de Stockolm, la capitale du pays, est de $1^{\circ} 91$ moins élóvée que celle du fort Moose.

Enfin la température du bassin méridional de la baie d'Hudson ne differe guère de la région dans laquelle se trourent Bergen et Christiania, les deux villes les plus populenses et les plus commerciales de la Norvègo, les deux grands centres de la partie la plus riche, la plus avancée et la plus peuplée de ce pays, Les chiffres suivants établissent cet énoncé : 


\begin{tabular}{|c|c|c|c|c|c|}
\hline . & Printemps & Et6 & Automne & Hiver & Annés \\
\hline 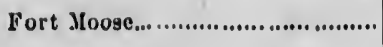 & $34 \bullet_{43}$ & $62 \odot^{\circ} 3$ & $30^{\circ} 69$ & $8^{\circ} 60$ & $35 \bullet 85$ \\
\hline Bergen ....................................... & $44 \circ 40$ & $58^{\circ} 60$ & $47 \circ 80$ & $36^{\circ} 30$ & $46 \circ 80$ \\
\hline 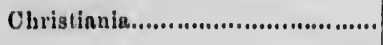 & $39 \circ 40$ & $69 \circ 70$ & $42 \circ 00$ & $22 \circ 80$ & $41 \circ 00$ \\
\hline Moyenne ........................... & $41^{\circ} 90$ & $50 \circ 15$ & $44^{\circ} 00$ & $20^{\circ} 40$ & $43 \subset 90$ \\
\hline 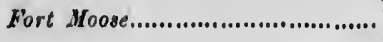 & $34 \bullet_{43}$ & $622^{\circ} 31$ & $39^{\circ} 60$ & $6^{\circ} 06$ & $35 \circ 85$ \\
\hline Difförence..................................... & -7.047 & $+3 \circ 16$ & -8021 & $-22 \circ 74$ & $8 \circ 05$ \\
\hline
\end{tabular}

C'est-ì-dire que la partie la plus chaude, la plus fertile et la plus habitée de la Norvège, telle que représentée par Bergen et Christiania, a une température de $7^{\circ} 47$ plus élevée que celle des environs de la baie James dans le printemps, de $3^{\circ} 16$ moins élevée en été, de $5^{\circ} 21$ plus élevée en automne, de $22^{\circ} 74$ plus haute en hiver et de $8^{\circ} 05$ plus chaude durant toute l'année.

Contrairement à ce qui a lien pour la vaste région avoisinant la baie James, la Norvège n'a qu'un sol montagneux et peu fertile, ce qui n'empêche pas que son territoire de 121,400 milles carrés est habité par une population de 1,742,000 âmes. L'étendue cultirée est de 2,840,500 acres et la population agricole excède 500,000 âmes. La production des céréales excède annuellement $15,000,000$ de minots. Quand l'agriculture donne de pareils résultats dans un pays dont le sol est montagneux, beaucoup moins fertile que celui de la région qui a le fort Móose pour centre, avec une température d'été de $3^{\circ} 16$ moins élerée et une atmosphère sonvent chargée de brouillard et d'inmidité, ce qui ne se voit pas au sud de la baie d'Hudson, il serait bien difficile de dire ponrquoi l'immense plaine qui aroisine cette baie au midi ne pourrait pas être cultivée avec profit et faire vivre une numbrense population agricole dans l'aisance et la prospérité.

En remontant daus l'intérieur des terres, c'est-à-dire en allant,vers le sud et le couchant, le climat de la contrée qui envoie ses eanx à la baie James s'améliore encore et la température moyenne est un peu plus élevée. Il n'a jamais été fait, que nous sachions, d'observations thermométriques régnlières dans cette région ; mais en consultant les donnnées elimatologiques que nous avons pour certaines localités situées dans le voisinage de la limite méridionale du bassin de la baie James, il est facile de voir que cet énoncé n'est pas contraire à la vérité. Une ligne tirée de l'est à l'ouest entre le fort Témiscamingue, sur l'Outaouais, et le fort Michipicoten, à l'extrémité nord-est du las Supérieur, suit à peu près la limite sud du bassin de la baie James. A ces deux points la température est comme suit : 
Latilude Longitude Printemps Ele Automne Hiver Anneo

Tomiscamingue............ $47010 \quad 79031 \quad 370^{\circ} \mathrm{O} \quad 65^{\circ} 2 \quad 400^{\circ} \quad 160 . \quad 380^{\circ}$

Mlchlpicoten ............... $47000 \quad 85000 \quad \frac{3706}{3706} \frac{5703}{0102} \frac{4102}{4006} \frac{1006}{1508} \frac{3802}{3804}$

Ces deux localités sont élevées de 660 pieds an-dessus du niveau de la mer, tandis que lo niveau moyen du bassin de la haie James nexcède guère trois cents pieds au-clessus de l'océan. Cette différence de niveau équirnut a un degré d'élévation dans la tempórature. Guyot prétend qu'une augmentation de hanteur de 330 pieds diminue la température d'un degré, d'où il snit qu'une diminntion correspondante de hauteur élève aussi la température d'autant. Pour Michipicoten, la température de l'étó est uu peu moins élórée: cela provient du voisinage des eaux du lac Supérieur, qui absorbent une partie de la chaleur atmosphérique et font baisser le mercure d'un ou deux dégrés. Mais cette influence ne se fait pas sentir au delà de la hauteur des terres et n'affecte aucunemont lo climat $d u$ bassin de la baio James. Dans la région du lac Supérieur, la température d'été est plus élevée au nord qu'au sud des Lanrentides.

En résumant toutes ces données, on arrive facilement à la conclnsion que les chiffres relatifs à la températ ure du fort Moose expriment exactement l'état de la température do la grande plaine silurienne égouttée par la rivière Abittibi, la rivière Moose et ses nombrenx rameaux, ainsi que par la rivière Albany. Cette plaine a une aire d'environ 125,000 milles, ou 80,000,000 d'acres carrés.

Dans la partie occientale, ou la valléo supérieure des rivières Nelson et Churchill, il n'y a qu'une localité-le fort Norway - où il a été fait des observations régulières sur la température. D'après Sir John Richardson, cette localité est située à $54^{\circ}$ de latitude et $: 8^{\circ}$ de longitude. Son élévation au-dessus du niveau de la mer est de 400 pieds. La tempórature moyenne résultant d'observations faites régulièrement durant sept ans, cie 1841 à 1847 , est de $26^{\circ} 2$ pour le printernps, $59^{\circ} 9$ pour l'été, $29^{\circ} 9$ pour l'antomne, $-3 \circ 7$ pour l'hiver, avec une moyenne annuelle de $28^{\circ} 1$ : Cette température n'est pas anssi farorable que celle $d n$ fort Moose aux opérations agricoles ; mais elle est suffisante pour faire mûrir la plupart des céréales et assez élevée pour permettre aux plantes gramincuses et aux légumes d'arriver à leur pleine croissance, ce qui est assez dire qu'en autant que cela dépend du climat, le contrée qui a lo fort Norway pour centre est tont à fait susceptible de culture et d'exploitation comme pays de paturage et d'élevage du bétail. Lo sol, du reste, se prèterait bien à ce geure d'exploitation.

$A u$ nord-ouest du fort Norway et dans les limites de la région qui nous occupe, il n'a jamais été fait d'observations thermométriques ; mais il est facile de constater, par induction an moins, que le climat n'est pas un obstacle à la culture et qu'eu général la température 
est assez élevéo pour faire mûrir toutes les céréales. Ontre la végétation forestière, qui suffirait ì établir co fair, il y a pour lo corroborer lo tómoignage de l'annlogie, ou plutôt de l'iủentité do la température des localités qui avoisinent cette région.

Le fort Chipewan, sur les bords du lac Athabaska, se trouve au nordouest, et le fort Cumberland, sur la Saskatchewan, au sud-est du plateau dans lequel les rivières Nelson et Churchill ont leurs" sources. Une ligne tirée entre ces deux forts partagerait presque ce platenu eu deux parties égales et passerait au nord-est do l'étendue dont le sol est assez fortile pour être cultivé avec profit. La tempórature moyenne de ces deux points extrêmes cloit étre à peu près cello do la région intermédiaire. Or, cu rapprochant-les observations thermométriques qui ont été faites avec soin dans ces deux localités, on forme le tableau suivant :

\begin{tabular}{|c|c|c|c|c|c|c|}
\hline Latitude & Longitude & Printemps & Ete & slutomne & Hiver & Annce \\
\hline .............58 $53 \circ 57$ & $102 \circ 20$ & $32 \circ 70$ & $62 \circ \mathrm{C2}$ & $33 \circ 04$ & -0017 & $33^{\circ} 20$ \\
\hline hippewnn .................880 83 & $1180^{\circ} 20$ & $22 \circ 76$ & $58 \circ 70$ & $31 \circ 89$ & $-3 \circ 34$ & $27 \circ 82$ \\
\hline
\end{tabular}

Ces données sont confirmées par l'expérience. Mgr Taché, qui con. nait personnellement le pays, et le professeur MaCoun, botaniste, qui l'a exploré pour la Commission Géologique, s'accordent à dire que le blé mûrit dans la belle vallée de la rivière aux Castors ainsi qu'au lac de l'ile à la Crosse. Or, il faut une température d'été de $60^{\circ}$ pour faire mûrir le blé, ce qui correspond avec le chiffre que nous avons formé en prenant la moyenne des températures de deux localités extrêmes, où il u été fait des observations thermométriques régulières. Cette concordance doit se reproduire pour les autres saisons, et alors nous arrivons à une température qui rend cette contrée parfaitement habitable comme pays agricole. L'étendue de bon sol arable qu'elle renferme est d'environ 12,000, milles ou 7,680,000 acres carrés, plus do cinq fois la grandeur de la province de l'Ile-du-Prince-Edouard, qui n'a qu'une superficie de 2,133 milles, ou 1,365,120 acres carrés.

La température de ce plateau correspond à celle de la Strède, dans la région d'Ostersund, où la température du printemps est de $34^{\circ} 03$, celle cle l'été de $56^{\circ} 11$, celle de l'automne de $34^{\circ} 57$, celle de l'hiver de $17=16$ et celle de l'anuée de $35^{\circ} 80$. La vallée de la rivière aux Castors se trouve sur les confins des prairies et reçoit une partie des rénts chands qui viennent du golfe du Mexique, par le sud; de l'onest, elle reçoit aussi une partie des vents qui viennent de l'océan Pacifique et arrivent jusqu'au lac de l'ile à la Crosse après avoir traversé les montagnes Rocheuses dans la dépression formée par la valléc de la rivière à la Paix. Ces diverses circoustances forment un climat comparativement peu rigourenx en hiver et dont la température en été est de deux dégrés plus élevée que celle de l'Angleterre. Est-il 
besoin d'ajouter que dans un pareil climat l'agr' ul+ure peut réussir complètement?

Sur les bords de la baie d'Hudson, à l'smbouchure des rivières Hayes et Charchill, le climat est trop rigoureux pour permettre l'établissement d'une population agricole. La température de l'été, c'est-d̀-dire la température moyenne, serait peut-être assez élevée pour faire mùrir l'orge et l'avoine, mais elle est sujette a des variations qui rendiaient fort précaire la croissance et la muraison de ces grains. Cependant, la chose n'est pas impossible, comme on l'affirme généralement sans tenir compte des taits. Il est certain qu'avec une culture soignée et dę variétés hâtives, on pourrait compter sur une assez bonne récolte. Pour atteindre leur pleine croissance et mûrir complètement, l'orge et l'avoin's n'exigent qu'une tempérnture d'éte de $58^{\circ}$; or, au fort York, cette température est de $59^{\circ} 79$, ce qui laisse u'c marge suffisante pour affirmer sans crainte que ces régions puis it produire l'orge et l'avoine. En 1876, le merrure n'est descendu que deux fois au-dessous du point de congélation durant les mois de juin, juillet et août: le ler juin à $27^{\circ}$ et le 16 ì $31^{\circ}$. Cela se roit assez sour znt dans les environs de Québec, ce qui n'empêche pas l'agriculture de réussir. D'un autre côté, la température s'est élerée jusqu'à $72^{\circ}, 74^{\circ}$ et $79^{\circ}$ dans le mois de juin; à $82^{\circ}$, $85^{\circ}, 87^{\circ}, 91^{\circ}$ et $99^{\circ}$ dans le mois de juillet, sans descendre plus bas que $39^{\circ} 5 ;$ à $70^{\circ}, 71^{\circ}, 76^{\circ}, 80^{\circ}$ et $86^{\circ}$ dans le mois d'août, arec une journée marquant $37^{\circ} 5$, ce qui montre que la tempérnture de ces trois mois n'est pas impropre ì la culturo des céréales les plus rustiques. Dans la dernière quinzaine do mai, la moyenne des plus basses températures a été de $36^{\circ} 0$ et celle des plus inautes a atteint $60^{\circ}$ 7. La moyenne générale de ces quinze jours a été de $47^{\circ} 4$, et le mercure a varié entre $25^{\circ}$ et $78^{\circ}$, les deux indications extrêmes. La température moyeune de la première quinzaine de septembre a été de $44^{\circ} 4$, aver des indications extrèmes de $32^{\circ}$ et $74^{\circ}$. Ces deux quinzaires allongent considérablemezt la saison agrico'e et donnent une température assez élevée pour préparer le sol dans le mois de mai, puis aider à la muraison des grains dans le mois de septembre.

La plupart des géographies et presque tous les récits de voyages on de voyageurs disent qu'au fort York la neige commence à tomber dans le mois de septembre et que lo sol en est courert avant la fin de ce mois. Les indications thermométriques que nous ronons de donner opposent un dementi formel à toutes ces fables: s'il tombe de la neige avant le $15^{\circ}$ de septembre, ce qui peut arriver accidentellement, elle ne peut toujours pas rester sur le sol, car la température est assez chaude pour la fondre. Du reste, s'il fallait condamucz comme impropres à la culture et inhabitables, les localités où il tombe de la neige dans le mois de septembre, il faudrait condamner à l'isolement plusieurs parties habitées de la province de Québec, notamment plusieurs paroisses du comté de Charlevoix: il y a cinq ola six ans, il est tombé lì̀, vers lo milieu de ssyptempre, une couche de neige de près d'un pied d'épais- 
seur,' ce qui n'empêche pas que ces paroisses sont encore habitées par une nombrruse population et produisent en abondance des céréales de toutes sortes.

Du reste, nous ne tenons pas à faire du fort York et du fort Churchill des centres agricoles; il y a plus au sud, dans les envirous de la baie James, des millions d'acres d'excellentes terres, jouissant incontestablement d'un très bon climat, et avant que toutes ces terres soient occupées, la température du fort York aura probablement le temps de s'améliorer. Nous constatons le fait que cette région jouit d'un climat habitable, pour le moins égal à celui d'Aarchangel, port fréquenté de la mer Blanche, seulement dans le but de montrur qu'on a beaucoup exagéré la righueur du climat de tout le bassin sud de la baie d'Hudson.

La région sud-est de la baie James est bien moins froide. Au fort Rupert, la température de l'hiver, au rapport de Richardson, est de $3^{\circ} 6$ et celle du printemps de $23^{\circ} 4$, avec $41^{\circ} 5$ pour le mois de mai et $34^{\circ} 8$ pour le mois d'octobre. Il n'a pas été fait d'observation pour les mois d'été ; mais en comparant cette localité à d'autres qui se trouvent dans la même position climatérique, il est évident que la température des mois de juin, juillet et août est pour le moins aussi élevée que celle du fort Norway, qui acruse pour ces trois mois une moyenne de $59^{\circ} 9$, c'est-à-dire $54^{\circ} 9$ pour juin, $63^{\circ} 5$ pour juillet et $61^{\circ} 2$ pour août. Le fort Rupert est plus à l'est ; mais il est aussi plus au sud et moins éleré que le fort Norway, en sorte que la température d'été derrait être plus élevée. De fait, on récolte actuellement de l'orge, des pommes de terre et toutes sortes de légumes sur le bords de l'EastMain, où la compagnie de la baie d'Hudson fait depuis longtemps l'élevage du bétail, autas.t de faits qui démontrent clairement que l'agriculture est praticable dans cette région et que la température d'été ne peut pas être beaucoup an-dessous de soixante dégrés. Le Dr Bell est d'avis que cette contrée pourrait faire un bon pays de pâturages et qu'à ce point de rue il a une raleur agricole incontestable.

$\mathrm{Au}$ fort Churchill, la température, sans ètre extrême en tout temps, est trop basse pour rendre pratiquement cette région habitable. Curtaines herbes, des légumes et les pommes de terre y vieunent assez bien ; mais la culture des céréales est impossible et h̀ raison de son climat, il faut ranger cette contrée parmi les régions incultes et inhabitables.

'Pour complétèr ces données, examinons un pen les renseignements que nous avons sur les phénomènes atmosphériques qui caractérisent ces régions. Si peu considérables, si maigres qu'ils soient, ces retrseignements sont nécesuires pour se former une idée complète du climat.

Les, phénomènes météorologiques, les variations de l'atmosphère n'ont été observées régulièrement qu'à deux localités, au fortYork et au fort Moose. Le résultat do ces obserrations est condensé dans les tableaux que nous domnons parmi les pièces annexbes. 
In consultant ces tableaux, on voit que les différents vents soufflent dans la proportion suivante:

$\begin{array}{ccccccccc}\text { N. } & \text { N.E. } & \text { D. } & \text { S.E. } & \text { S. } & \text { S.0. } & \text { 0. } & \text { Y.O. } & \\ \text { Fort-York .........21.83 } & 10.40 & 10.10 & 0.41 & 15.25 & 7.15 & 11.72 & 17.75 & \text { pour } 100 \\ \text { Fort-Noose........15.56 } & 16.96 & 2.29 & 2.99 & 5.88 & 21.84 & 13.07 & 20.35 & \text { “ “ }\end{array}$

Dans la partie sud du bassin de la baio d'Hudson, les reuts qui apportent de la chaleur sont cenx qui sonfflent du sud, du sud-ouest et de l'ouest. La proportion de ces rents est de 34.12 pour $100 \mathrm{au}$ fort York et de 40.79 pour 100 au fort Moose, ce qui accuse une différence de 6.67 pour 100 en faveur de cette dernière localité. Ce sont aussi ces mêmes rents qui apportent la pluie et la neige, de sorte qu'il doit en tomber plus au fort Moose qu'au fort York. Daus la saison douce, ces vents transportent l'humidité qu'ils premneut dans les lieux où ils originent et en arrivant dans les couches d'air plus froides de la baie d'Hudson, cette humidité se condense et tombe en pluie. La quantité de pluie est de 10.54 ponces au fort Moose et de 47.50 pouces au fort York, avec 42 jours pluvieux dans la dernière localité, èt seulement 38 dans l'autre, où les orages sont biez moins abondants.

Au fort Moose,ce sont les vents de nord qui prévalent durant les mois d'avril, mai et juin. L'air, se réchauffant plus tôt que dans les régions situées au nord, est déplacé par les courants froids venaut dn nord et ces courants constituent les vents dominnuts, ce qui retarde la végétation et abaisse la température naturelle. Cependant, même dans le mois de mars, le thermomètre marque parfois jusqu'à $45^{\circ}$ et $50^{\circ}$, lorsque la température n'est pas abaissée par les vents.

Ou sait que daus les latitudes élevées, comme au fort Moose, le soleil communique beaucoup plus de chaleur à la cerre que dans les régious plins rapprochées de l'équateur, à raison de la plus grande longeur des jours. En été, la durée du plus long jour est de 16.3 heures au 50e degré de latitude, de 17.3 heures au 55e et de 18.7 heures au $60 \mathrm{e}$. On comprend facilement que ce séjour prolongé du soleil à la surface de la terre élève considérablement la température, sans compter que les couches d'air diminuant d'épaisseur en allant au nord, elles absorbent moins la chaleur solaire, qui arrive presque toute à la terre. Ces faits expliquent jusqu'à un certain point li haute température d'été de la région située au sud de la baie James, or jil n'y a pas de grandes masses d'ean comme an nord pour absorber uno partie de la chaleur que le soleil répand dans ces contrées.

Dans la région de la baie James, les saisons se ressentent nn peu des conditions climatériques particulières à co pays. Le printenıps est un peu tardif, comparé à celui de la Rivière-Rouge. Le sol se découv'e assez à bonne heure-généralement daus le mois d'avril et souvent arant le quinze de ce mois-mais la température de l'atmosphère ne s'élève que lentement. Les glaces qui recouvrent la baie absorbent une partio de la chaleur solaire pour se fondre, et quand olles sont 
disparues, les vents de nord qui soufflent assez souvent à cette époque refroidissent considérablement l'air. Ces vents alternent avec le calme et les vents chauds du sud, ce qui produit une température variable et parfois un peu désagréable. Dans la première quinzaine d'avril, lo mercure descend quelquefois jusqu'à n'uf et même cinq degrés et dans l'antre partie de ce mois il varie entre ringt et soixałte. En 1878, le thermomêtre a inarqué durant les premiers ciuq jours de mai $62^{\circ} 9$, $65^{\circ}, 58^{\circ}, 61^{\circ} 1$ et $54^{\circ} 9$ pour les plus hantes températures, puis $46^{\circ} 1,40^{\circ} 9,42^{\circ} 1,43^{\circ}$ et $37^{\circ} 6$ pour les plus basses. Lo mercure est descendu à $19^{\circ}$ le 13 et le 14 , mais n'a pas haissé au-dessous du point de congélation le reste du mois, excepté le 18, où il s'est arrêté à $30^{\circ} 9$. En revanche, la plus haute température des deux derniers jours a atteint $66^{\circ} 1$ et $76^{\circ} 5$.

Ces températures extrèmes ont beaucoup d'analogie avec celles de la prorince de Québec à la même saison. Jans la partie est de la province, il est rare qu'il ne gèle pas plusieurs fois durant le mois de 'mai, ce qui n'empêche pas les cultivateurs de commencer leurs semailles et de se lirrer sans difficulté aux traranx des champs. Et nos vents de nord-est, qui souffent presqu'en permanence pendant ce mois, sont pour le moins aussi froids et aussi désagréables que les vents de nord du fort Moose et de la baie .James.

L'été est très bean et comparativement très chaud dans la partie sud du bassin de la baie d'Hudson. Au fort Moose, la température des premiers jours de juin forme souvent des écarls considérables, variant entre $78^{\circ}$ pour maximum le premier et $26^{\circ} 9$ pour minimum lo six, ainsi que cela est arrivé en 1878; mais à partir dn quinze, le thermomêtre se tient généralement anx environs de $60^{\circ}$ et indique parfois jusqu'ǹ $92^{\circ}$. En juillet, le mercure ne descend pas au-dessous de $4 i^{-}$, ce quı n'arrive que par exception, et sur le midi il est presque tous les jours au-dessus de $70^{\circ}$, atteignant parfois jusqu'à $91^{\circ}$, même dans les premiers jours du mois. Ia même température se maintient durant la première quinzaine d'août, et durant tout co mois les écarts entre les températures extrèmes sont bien moins considérables que durant le précédent. En 1878, la plus basse indication du thermomêtru a été $34^{\circ} 0$ le 28 août.

Durant toute cette saison, l'atmosphère est claire et l'air calme. Les vents $d u$ sud et de l'onest, qui sont les plus fréquents, ne sont quo des brises donces qui ne font pas baisser le thermometre. Il ne tombe que pou de pluie: le registre tenu on 1878, au lort Moose, mentionne trente-huit jours pluvieux pour les trois mois : 9 en juin, 12 en juillet et 17 en août. Mais ces pluies sont légères et de peu de durée, puisque le registre que nots venons de mentionner n'indique que 10.54 pouces : $1.6 \dot{t}$ en juin, 2.79 en juillet et 6.11 en août. Durant ces trois mois, il n'y a ci que quatre orages accompagnés de tonnerre, ce qui proure claire'nent la pureté de 'atmosphère. Enfin, le elimat d'été des envirolis de la baie Jannes est excellent sous tous les autres rapports, et nous avons vu ailleurs que sa température est plus élevée, 
que celles des régions les plus peuplées et les plus productives de l'Europe.

L'automne est comparativement beaucoup plus chaud que le printemps, dans les elıvirons de la baie James . L'eau de la mer ne perd que très lentement la chaleur qu'elle a absorbée jurant l'été et neutralise les effets des autres causes qui tendent à abaisser la température. En 1878, le mercure n'est descendu au-dessous de 0 (à $31^{\circ} 1$ ) que le 27 septembre et jusqu'au 15 de ce mois les plus basses indicatious ont généralement varié entre $40^{\circ}$ et 5()$^{\circ}$. Il est remonté a $78^{\circ} \mathrm{le}$ 4 et à $64^{\circ}$ le 28 . En octobre, la première gelée a eu lieu le 16 et le mercure est redesceudu au-dessous de 0 huit fois jusqu'à la fin ciu mois. Les plus hautes indications ont été $66^{\circ}$ le $2,60^{\circ} 5$ le 10 et $63^{\circ} 6$ le 21 . Comme on le roit, les gelées ne commencent pas avant lo quinze, f t inême après cette date, il y a encore plusieurs journées de chaleur, ce qui correspond à la température des plus belles parties de laprovince de Québec. Le registre pour 1878 mentionne pourseptembre: un orage accompagné de tonnerre, 19 jours pluvieux et 5.46 pouces de pluie ; pour octobre, 1.74 pouees et 14 jours de pluie, 5 jours et 3 pouces de neige ; pour novembre, 3 jours plnvieux, puis 19 jours et 25.5 pouces de neige. Comme on le voit, l'automne est aussi bean sur les bords de la baie James que sur ceux du Saint-Laurent et la température du mois de septembre, surtout, n'oftre rien qui puisse nuire d'une manière sériense aux opérations agrieoles. En 1878, la première neige est tombée le 15 octobre et il n'y en arait encore que quatre pouces sur le sol le 4 novembre, deux jours après que la glace fût prise sur la riviere Moose, ce qui correspond encore avec l'occurence des mêmes phènomènes dans notre province.

L'hiver, proportion gardée, est beaucoup plus rigoureux que les autres saisons. En novembre, la température baisse parfois jusqu'à $4^{\circ}$ et ne s'élève pas beaucoup au-dessus de trente-deux. Les froids extrêmes de décembre vont jusqu'à-18 ${ }^{\circ}$ et-20 ${ }^{\circ}$ et en 1878 le thermo. mètre n'a douné qu'une indication-32०9-au-dessus du point de cougélatiou. Janvier est généralement le mois le plus froid. Le regis: 're que nous arons sous les yeux indique pour ce mois trois extrênes de froid :-35 9 le $5,-31^{\circ} 9$ le 7 et-31 ${ }^{\circ} 4$ le 23 . La plus haute température, $32 \circ 7$, a été enregistrée le 19. Les températures extrêmes de té. rrier ont été $-21^{\circ} 7$ le 9 et-42 ${ }^{\circ} 5$ le 26 . Il y a en deux jours pluvieux et 0.65 pouces de pluie, ptiis 12 jours et 15.8 pouces de neige en déeembre. En janrier, il a été enrégistré 16 jours et 9.6 pouces de neige, puis 10 jours et 8.1 ponces de neige pour février, en qui fait pour les trois mois 38 jours et 33.5 ponces de neige. Nons en arons heancoup plus dans les envirous de Québec. Durant ees trois, mois ce sont presqu'exelusivement les rents d'ouest qui souffent, principalenant celui du nord-ouest, ce gui est un point de ressemblance avec nos hivers de la province de Québec.

On a géuéralement me idée très erronée sur la rigueur de l'hiver dans la contrée que nous étudions. Jugéant du froid par les indicacations thermométriques, on se figure que ce froid est intolérable, 
tandis qu'il est réellement moins rude que la température de nos hivers de la province de Québec. C'est l'huraidité de l'atmosphère qui rend le froid insupportable, en le rendant beaucoup plus pénétrant. Quand le temps est sec, comme on dit vulgairement, on ne souffre guère du froid, même quand le mercure deseend bien bas au-dessous de 0 , au lieu qu'on souffre beancoup lorsque le temps est humide et le mercure beaucoup plus haut. Dans une atmosphère sèche et pure, le thermomètre peut descendre au-dessous de zéro sans que la gelée se produise. D'après les observations du commissaire Wheelock, du Minnesota, la température peut baisser-et. dans cet état a souvent baissé-jusqu'à $20^{\circ}$ sans affecter visiblement la végétation, quand l'air est sec. L'air sec et pur est mauvais conducteur du froid et il faut que le thermomètre descende extrêmemert bas pour qu'on souffre $\mathrm{du}$ froid quand l'atmosphère est sèche. C'est précisément ce qui a lieu dans la région de la baie James, où le ciei est tonjours clair en même temps que l'air est sec, en hiver, et c'est ce qui explique pourquoi, ld̀ comme dans les prairies de la Rivière-Rouge, avec des indications thermométriques extrêmes, on a une ttmpérature d'hiver très-supportable, pas plus froide en réalité et beancoup plus agréable que celle des hivers des autres provinces du Canada.

Nous croyons aroir démontré que le climat n'offre aucune objection à l'établissement des plaines fertiles qui envirounent la partie méridinale du bassin de la baie d'Hudson, notamment la baie James. S'il fallait ajouter d'autres preuves à l'appui de cet avancé, nous pourrions constater que la saison libre, c'est-à-dire l'intervalle durant lequel il ne gèle pas, est aussi longue et même plus longue que dans plusieurs des parties actuellement habitées du Canada. En 1878, au tort Moose, la dernière gelée du printemps a eu lieu le 21 mai et la première gelée d'antomne le 27 septembre, ce qui laisse un intervalle libre de 129 jours entre ces deux dates. En consultant les rugistres météorologiques pour les deux ou trois dernières années, nous constatons que cet intervalle libre de gelées n'a été que de cent vingt jours à Winnipeg, du 14 mai au 15 septembre, ce qui fait une différence de neuf jours en faveur de la région de la baie James. Pourtant, il serait difficile de soutenir aujourd'hui que Winnipeg ne jouit pas d'un beau climat agricole!

En plusieurs endroits habités de la province de Québec et de la partie nord de celle d'Ontario, cet iutervalle libre est encore plus court qu'à Winnipeg et surtout au fort Moose, ce qui n'empéche pas l'agriculture de réussir parfaitement dans ces contrées-là.

Citons un dernier fait. Pour la saison agricole, c'est-à-dire les six mois compris entre mai et octobre inclusivement, la moyenne de la température an fort Moose est de $54^{\circ} 48$, telle qu'enrégistrée en 1878. En présence de ce fait, scrait-il possible de croire que le climat do cette région peut offrir le moiudre obstacle à l'agriculture, quand il est constaté par l'expérience qu'en Amérique comme en Furope le blé, qui est de toutes les céréales la plus délicate à cultiver, se contente d'une température moyenne de $60^{\circ}$ pour les trois mois d'été ? 
Qu'on motte les préjugés et l'ignorance de côté, et l'on sera forcé d'admettre qu'au point de vue du clinat, comme sous le rapport du sol, la plus grande partie du bassin méridional de la baie d'Hudson est une des parties les plus habitables du Canada. Il en sera do cette région comme il en a été de celles de la Rivière-Rouge et de la Saskatchewan: quand on la connattra bien, on se disputera au poids de l'or les belles terres qu'elle renferme et l'on s'occupera bien moins des indications $d u$ thermomètre que des richesses inépuisables recelées dans son sol.

\section{CHAPITRE V}

FORETS-I.EURS IAIMITES-PARTICULARITES-ESSENCES DOMINANTES PARTIL 3 SUSCEPTIBIES D'EXPLOITATION-FACILITK POUR IAA

DESCENTE DES BOIS

Toute la région que nous esquissons est couverte de forêts, à l'exception d'une petite lisière située au nord d'une ligne tirée entre l'embouchure de la rivière Nataskopa et la partie sud-ouest de la baie d'Ungava, dans le Labrador. Les dimensions des arbres appartenant aux différentes essences ligneuses sont plus ou moins considérables, suivant la uature du sol et du climat ; mais la forêt, quelle qu'elle soit, recouvre partout le sol.

Dans les forêts du bassin méridional de la baie d'Hudson, les es. sences ligneuses offrent des particularités qu'il est intéressant de no. ter. Ainsi des espèces qui recherchent ailleurs des terrains murécageux et froids, croissent de préférence, dans ces régions, sur les terrains secs et montueux. Dans le sud de la région de la baie James et dans presque toutes les autres parties du Canada, l'épinette rouge, le sapin et l'épinette blanche qui, dans les autres régious, ne poussent que dans les marécages ou les terrains humides et froids, ne se voient généralement, dans le nord de la contrée de la baie d'Hudson, que daus les terrains secs et an peu élevés, principalement sur les hauteurs des endroits montueux. En plusieurs loc:alités, le cèdre blanc et le pin blanc semblent avoir la même tendance $\grave{z}$ rechercher les terrains secs et élevés.*

La zone occupée respectivement par chacune des différentes espèces n'est pas entièrement limitée par la moyenne de la température annuelle. L'humidité de l'atmosphère entre ponr beaucoup dans la déterminatiou de ces limites. Si grand que soit l'écart entre les froids et les chale:urs extrêmes, la végétation forestière se développe et la zone de certaines espèces s'agrandit du moment que ces espèces rencontrent les conditions atmosphériques qui leur conviennent. Aiusi

- Dr Robert Bell-Rappórt Geologique pour 1879-80, page 40c et séq. 
l'épinette blanche et noire qui, sur les bords de la baie d'Hudson, descend considérablement, rumonte graduellement au nord, a l'est et à l'ouest, en s'éloignant des rivages de la mer et en atteignant les terrains plus élevés. Dans la partie nord-onest du Labrador, la limite des zoues forestières incline vers le nord à mi-distance entre les rivages de la baie et du détroit d'Hudson. La végétation forestière est arrêtée sur les bords de la mer par l'influence défnvorable qu'exerce le voisinage de l'eau, tandis qu'elle se développe dans le plateau plus élevé et plus sec qui se trouve an miliè. Le même phénomène a été constaté dans la contrée qui s'étend à l'ouest de la baie d'Hudson. Sous une température plus haute, la régétation souffre plus du froid dans une région humide, qu'elle n'en souffre dans les endroits où l'air est sec, mais la température plus basse. In amollissant l'écorce des arbres, l'humidité les expose beaucoup plus anx attaques du froid et de la gelée, qui les péuètre beaucoup plus facilement. L'air sec, au contraire, est maurais conducteur du froid, affermit et endurcit l'écorce des arbres an lien de l'amolir et favorise la végétation dans des localités où le thermomètre indique des froids extrêmes. C'est pourquoi la limite septentrionale de toutes les zones forestières s'élève vers le nord en s'éloignant des bords un pen humides de la baie James pour gaguer les plaines plus sèches qui s'étendent de chaque côté à l'est et à l'ouest. C'est aussi ce qui explique pourquoi certaines essences lignénses, dont la limite nord se trouve au sud de la région de la baie d'Hudson, telles que le pin et l'orme blanc, qui ne croissent pas dans la coutrée humide du lac Supérieur, se trourent de l'autre côté des Laurentides et croissent bien dans lo plateau où les rivières Abittibi, Missinaibi et Albany out leurs sources. Sur les bords de la Missinaibi, l'orme blanc crott à 130 milles au nord de la limité générale de la zone qu'il occupe.

Donnons maintenant quelques détails sur les quinze ou seize espèces de bois qui composent les forêts de la partic sud de la baie d'Hudson. La limite nord de chacune de ces espèces est indiquée sur la carte."

... EPINETTE MLANCHE, EPINETTE DE MER, EPINETTE SEULE (abies alba, MICHAUD). Les sauvages l'appellent épinette hlanche pour la distinguer de l'épinette noire. Cette espèce est celle qui s'avance la plus loin vers le uord. A l'embouchure de la rivière à la Baleine et de la Grande-Rivière, ou rivière George, eet arbre est asse\% gros pour être utilisé comme bois de service, mais son tronc est court et souvent gâté par les nœuds. Cependant, le Dr Bell a vu an fort George une quantité de billots faits de ce bois qui mesuraient vingt-quatre pouces de diamètre. Ces billots avaient été coupés dans la vallée de la rivière George, à quelques milles de la mer. Sur les bords de la baie d'Hudson, cet arbre disparatt aux environs du golfe Richmond, vers $57^{\circ}$

- Ces indications et ces renseignements sont pris sur la carte publibe par le I)r Boll et dans l'excellente étule qui accom jagne cutte carte. 
de latitude ; mais de l'autre côté de la baie, il monte plus au nord et on lo trouve jusque sur les bords de la rivière au Loup-Marin, par $59^{\circ}$ de latituds. Dans les environs de la baie James, puis entro cette baie et le lac Supérieur, il atteint des dimensions qui le rendent propre à l'exploitation comme bois de commerce, et rnême sur les bords des rivières Hayes et Nelson, le Dr Bell a vu des billots d'épinette blanche bons, sains, mesurant deux pieds de diamètre. C'est de beaucoup le bois le plus commnn dans les forêts de cette région, où il crott dans les espaces de sol riche, ou sur les bords des lacs et des rivières.

2. EPINETTE NOIRE, EPINETTE DOUIBLE (abies nigra, POIR). Cet arbre a la même limite nord que l'épinette blanche; mais on le trouve surtout dans les terrains marécageux et froids ou sur les hauteurs des collines. Il est plus petit que son congénère et, en général, n'a pas assez de développement pour aroir quelque valeur comme bois de service. Dans les endroits où il est le plus beau, on pourrait s'en servir tout au plus pour faire des traverses de chemin de fer.

3. MELEZZE, TAMARAC, EPINETTE JROUGE, JUNIPER (Larix americana, MICHAUX). La limite septentrionale de cette espèce est peu au sud do celles des deux précédentes, et comme l'épinette blanche, elle atteint son plus grand développernent sur les terrains secs et élevés ainsi que dans les terres riches qui avoisinent les riviêres. Cet arbre crott aussi dans les terrains humides et marécageux, avec l'épinette noire ; mais là il est petit et sans valeur. Il ponsse à perfection dans la contrée située au midi de la baie James, où il atteint des proportions qui en font un bon bois de service et de commer..

4. SAPIN BLANC, SAPIN, PIN ARGENTÉ (Abies balsamea, MARSHaLI.) On le trouve dansles environs de la baie James ; mais sa limite septentrionale ne dépasse pas le sud-onest de la baie d'Hudson, passant entre le fort Severn et le lac à la Truite pour atteindre le confluent de la Shammattawa avec la rivière d'Acier. A ce point, la ligne fléchit. au sud-ouest et traverse la rivière Nelson à la décharge du lac Sipiwesk, pour courir ensuite au nord-ouest jusqu'au fleuve Mckenzie, qu'elle atteint vers $65^{\circ}$ de latitude. Au sud-onest de la baie d'Hudson, cet arbre ne crott que dans les terrains fertiles et chauds; on ne le trouve nulle part dans les régions froides et marécageuses. En beatcaup d'endroits, il a des proportions qui le rendent utile comme bois de service, notamment dans la région des rivières Nelson et Chur. chill, où il mesure jusqu'à quatre pieds de circonférence daus les envirous du lac de l'Ile.

5. PIN DE BANK, PIN NAIN, CYPriss (Pinus tunlsiana, LAMB3.)-Cet arbre ne se rencontre pas an nord d'une ligne partant du lac Mistassini, passant à cent mille: de la baie James et courant ensuite au nord-ouest jusqu'au fleuve McKenzie, qu'elle traverse dans le cercle arctique. Petit et rabougri aux extrèmes de la zone qu'il occupe, ce pin prend les proportions d'un bon urbre forestier au centre de cette zone. Sur les branches sud de la rivière Albany, le Dr Bell a vu do petites forêts de ces arbres, mesurant soixante-dix pieds de hauleur et deux pieds de diamètro à la hanteur de la sonche, et les troncs do 
ces arbres sont droits et sans branches jusqu'à une hauteur de vingt ou trente pieds, ce qui indique assez que cette essence est précieuse comme bois de service.

6. PIN BLANC (Pinus strobus, L.) On trouve des arbres de cette espèce, de bonnes dimensions, dans la vallée supérieure des principaux tributaires de la rivière Moose. Antrefois, il se rencontrait bien plus au nord, dans cette région, mais les forêts qu'il formait ont été détrnites par le fen. Sans être abondant, on le tronve en forêts assez considérables dans la région que nous venons d'indiquer.

7. CEDDRE BIAANC, CEDRE (Thurya occidentalss, L.) La limite nord de cet arbre part dn fort Rupert, passe daus le voisinage du fort Moose, traverse la rivière Albany à peu de distance de la mer, se prolonge jusqu'à soixante-quinze milles au sud-onest dn lac à la Truite et de là court au sud jusqu'à la tête du lac Winnipeg. Il pousse généralement dans les terrains bas, et en beancoup d'endroits il a les proportions voulues pour ètre utile comme bois de serrice.

8. CÈDRE ROUcie (Juniperus virgiana, L.) La variété de ceite espèce, le guniperus humilis (Hook), crott jusqu'à l'embouchure de ia rivière Nelson, dans les endroits secs et rocheux, ainsi que le long des lacs et des rivières, dans les régions boisées. Cet arbre n'a aucune valeur.

9. PEUPJiER BatsaMIFERE, ARBRT à COTON (Populus balsamifera, $\mathrm{x}$ ) Cet arbre ne va pas an delà d'une ligne tirée entre le fort Churchill et $65^{\circ}$ de latitude sur le McKenzie. Le long de toutes les rivières qui se jettent dans la baie James et la partic sud-onest de la baie d'Hudson, il pousse en abondance, mais n'atteint pas de grandes dimensions ; il forme de belles forêts sur les bords du lac Vert et la rivière aux Castors, où il est assez long pour être scié en planches.

10. TRkMBLE, PEUPIIER OOMMUN (Populus tremuloides, MICHAUd.) Sans aller aussi loin verś le nord que l'espèce précédente, le tremble pousse dans les environs de la baie James ; mais du côté ouest, on ne le trouve qu'à nue certaine distance du rivage de la baie d'Hudson. Il forme d'abondantes forêts dans les environs du lac Vert et le long des rivières qui conduisent de ce lac à celui de l'1le â la Orosse, où il atteint des dimensious considérables. Dans le Nord-Ouest, on s'en sert comme bois de chanffage, de préférence aux conifères, parce qu'il fait moins d'étincelles et n'expose pas les Sauvages à brûler leurs huttes d'écorce.

11. BOULEAU, whitebirch des Anglais, (Betula papyracea, ART.) La limite septentrionale de cette essence est à peu près la même que celle du tremble. Ce bois pousse presque partont dans la région de la baie d'Hudson, sans former de forèts ; il crott parmi les autres arbres. Il a jusqu'à dix et douze pouces de diamètre, et dans la région qu'il occupe il est assez gros pour que son écorce puisse servir à la confection des canots, ce qui fait qu'on l'appelle aussi bouleau i canots.

12. BOULEAU JAUNE (Belula excalsu, ART.) On trouve quelques arbres de cette espèce dans les environs du lac $A$ bittibi, mais pas plus au nord, où sa limite ne dépasse pas $40^{\circ}$ de latitude.

13. FRÊNE NOIK, FRENE DE SAVANE (Fraxinus Sambucifolia, L.) 
Le Dr Bell n'a pas rencontré cetie essence an nord du portage SaintPierre, sur la rivière Missinaibi. Ce bois crott le long de tous les tributaires, ou plutôt des branches de la rivière Moose, mais n'atteint pas de grandes dimensions. Il ne peut guère avoir d'utilité comme bois de service.

14. FRENe DE MONTAGNe, Rowan (Cyprusamericana, DE o.) La limite nord de cet arbre ne dépasse pas la baie James, n l'est ; mais en allant vors l'ouest cette limite monte lo long do la rivière Nelson jusqu'd quatre-vingt milles du lac Winnipeg. Il est de bonne taille dans la rógion de la baie James, mais devient plus petit vers le nord-ouest, notamment au lac de l'Jle, sur la rivière Shammattawa, et à la chute de la Vase-Blanche, sur la rivière Nelson, où il croit en assez graude quantité.

15. KRABLE DE MONTAGNe (Acuspicatum, LAMB.) Cet arbre so rincontre dans la région de la baie Janes et jusqu'au lac de l'Hle, sur la rivière Nelson, ce qui paratt être sa limite nord.

Telles sont les essences ligueuses qui constituent d'une manière d̀ pen près exclusive les forêts de la région méridionale du bassin de la baie d'Hudson. Lus plus répandues sont les conifềres, principalement l'épinette blanche, le tremble, le peuplier et le bouleau. Pour donner une idée de ces forêts, nous citons quelques extraits des rapports publiés par les voyageurs ou les spécialistes qui ont exploré ou parcouru ce pays.

Le bois (sur les bords de la rivière au Cariban), qui est petit et de pauvre qualité, se compose priueipalement d'épinette ; mais on trouve souvent $d u$ tremble, $d u$ peupliur balsamique, du bouleau et $d u$ pin de Bank. A un certain endroit, pas loin de la décharge du lac, j'ai vu beaucoup d'épinettes rouges: mais, quoique ces arbres fussent longs, ils avaient peu de grosseur.

(Rapport Gélogique pour $1879-80$, page 14 c.)

Rivière aux Castors.- "La rivière anx Castor est un beau pays pour former des établiesements. Le sol est bou et fort, bien boisé, et même fortement boisé, de magnifiques épinettes (pins) et d'antres bois de valeur." (Peace River-Journal of chief factor A. MacDonald, page 56.)

"De ce point à Carlton, il n'y a qu'environ cent milles à parconrir à travers un beau pays uni et bien boisé... La forêt d'épinette (pine forest), d'uprès Simpson, qui a parcouru toute la distance en hiver de Carlton au lac Vert, commence à la latitude $53^{\circ} 30^{\prime}$ nord, un peu plus qu'à la moitié du chemin pour se rendre au lac Vert." (Idem, pages 60 et 61.$)$

Du luc de l'Ile-a-la-Crosse au lac Vert.- " Lo long de la rivière, le terrain s'élève graduellement, mais il n'y a pas de changement dans l'apparence du pays. Des bords à pente donce, couverts de forèts de jeunes peupliers, à travers lesquels se trouvent quelques pins de Bank, rariés par une forèt d'épinette..." (Macoun.-Rapport déolog?que pour $1875 \cdot 76$, page 180 .)

"Nous suivons la branche ganche et remarquons de suite une 
amélioration dans l'apparence du pays. De belles forêts de trembles et de penpliers bordent la rivière des deux côtés, et tonte cette région semble couverte de la mème espèce de bois." (Idem, page 180.)

"Des deux côtés (du lac Vert) le terrain s'élève graduellement des berges de galets, et il est recouvert par une épaisse forèt do grands trembles et de grands peupliers, ce qui indiqno du bon terrain et nn sol productif." (Idem, page 181.)

Riviere Nelson.- "Aux environs (du fort Norway) le pays est rocheux, accidenté par des buttes et des marais, bien boisé de pins, d'épinettes et de cèdres (probablement), et il y a beaucoup de terrain sec. Les arbres, en grande partie, sont des pins (pines) de bonne qualité et de différentes espèces, des épinettes et des bouleaux, blanes et rouges, ou mẻme noirs, si je me rappelle bien. Il y a, dans tous les cas, une bonne sucrerie de boulenux (birch sugary) dans le voisinage... Il pent y avoir de l'érable, probablement qu'il y en a, c'esta.dire de l'espèce qu'on trouve dans le nord. Ies arbres sont d'une assez bonne taille, un billot de dix-sept ponces de diamètre au bout; au petit bout, n'étant pas chose impossible à tronver." (Peace RiverJournal of chief factor A. Mc.Donald, page 51.)

"Le paye avoisinant les lacs Travers et Walker est généralement uni. Le terrain paratt joliment rocheux, mais à différents endroits, sur les bords de ces laes, on tronvo beaucoup de sol glaiseux. La forêt, qui est presque tonte verte ou n'a pas été atteinte par lo feu, so compose d'épinettes, de pins de Bank, d'épinettes rouges, de sapins, do bouleaux, de trembles et de peupliers, avec des saules do deux ou trois espèces... et rarement des frènes de montague." (Rapport Geologique pour $1879-80$, page $6 \mathrm{c}$.)

"On dit que le peuplier et l'épinetto rouge disparaissaient entre la rivière Nelson et la partie inférieure de la rivière Churchill, au lieu que l'épinette noire se voit à quelque distance audelà de la rivière au Loup-Marin. L'épinette blanche, d'une assez bonne grosseur pour étre employée dans les constructions, se trouve sur les lles et les terrains plats le long du cours inférieur de la rivière Nelson... Une petite étendno de bois a été conservée stir le côté onest de l'lle Ross, à l'encroit où la rivière de l'Ouest entre dans lo lac des Gros-Roseaux, et là beaucoup d'épinettes blanches mesurent trois pieds de diamètre. Les endroits même les plus rocheux supportent une torêt d'arbres assez gros pour avoir de la valeur et être employés pour beaucoup de fins, si ce grand territoire vient jamais à être habité par des hommes civilisés.

"Les forêts et la flore de la région de la rivière Nelson, en général, indiquent un elimat plus donx que celui des localités situées dans une position correspondante de l'autre eôté do la baie d'Hudson... La terre parait facile à défricher, et considérant la quantité illimitée de bois de construction, de chauffage, \&c... cette région offre aux im. migrants des avantages qué u'offre pas la plus graude partie des régious de prairies situées plus à l'ouest." (Dr Bell-Rapport Geologique pour 1877-78, page 29 et $80 \mathrm{cc}$.) 
“La région traversée en suivant la route de la rivière à l'Herbe, entre son embonch ure et le lac Sipiwesk, présente généralement nuo surface ondoyante. Le terrain est ordinairenent glaisenx, et lo sol souvent bon. Ici et à quelques autres endroits le long da cetto ronte, la forèt a passé au feu ; mais dans cette région lo bois, pour la plas grande partie, est vert et de forte venue, les épinettes meucurant parfois au delà de six pieds de circonférence." (Dr Bell, Rappont Génealogique pour 1878-79, pages 28 et 39c.)

"Region cles lacs du Genou, Oxfurd, Sud et de l'Ile"-Dans toute cetto région, l'épinet te est l'essence la plus abondauto. Vienuent ensuito par ordre, lo tremble, le boulean blane, l'épinette rouge, lo peuplier et le pin de Bauk. Eu beauconp d'endroits, l'épinette atteint de très bonnes dimensions et est einployée sous forme de billots et de poutres dans les constructions de bâtisses. On la scie aussi on planches et en madriers pour l'employer dans toute sorte d'ouvrages de menuiserie. L'épinette rouge et le pin de Bank ont quelquefois vingt ponces de diamètre. Lue sapin est commun et de bounes dimensions aux envirous dı lac de l'Ile, où quelques-uns de ces arbres mesurent jusqu'à quatre pieds de circonférence; mais il est rare an lac God, et on n'en voit que peu et de petites dimensions an lac du Genou. En allant au sud, on rencontre pour la première fois le frêne de montrgne sur les bords du lac de l'tle. L'érable noire? (Groundmaple ?) n'a été v ue qu'su sud de ce lac." (Idem, page 38c.)

Rivière Kenogami. "La rivière Anglaise ou Kenogami coule à travers un terrain uni partout, depuis le lac Long jusqu'à la ririère Albany... Le sol, sur le haut des bords de la rivière et jusqu'd nne certaine distance, a paru très bon dans la plupart des endroits où il a été examiné. Le bois se compose en grando partie d'épinettes blanches, de sapins, de cèdres blancs, d'épinettus rouges, de bouleanx blanes et de trembles. Quelques-unes deis plus grosses épinettes blanches et rouges mesurent de cinq à six pieds de circonférence à cinq pieds du sol, mais en moyenne le diamètre des plus gros arores est d'environ dix-huit pouces." (Dr Bell, Rapport Géolomique pour 1870-71, page 340.)

" En arrière de ces lacs (Wa-wong et Fluming, ì la tête de la rivière Kenogami), la surface du pays est onduleuse et le sol se compose généralement d'un sable léger et gravelenx. La forêt se compose de bouleau, de tremble, d'épiaette rouge, d'épinette blanche, de sapin, de cèdre blanc et de pin de Banks, ou "cyprès" : beaucoup de ces arbres sont assez gros pour avoir de la valeur comme bois de construction. On dit que des terrains semblables à ceux qui viennent d'être décrits s'étendent dans le voisinage do la hauteur des ierres, à l'onest, juśqu'au lac Nipigon et à l'est jusqu'au fort Nouveau-Brunswick, sur la rivière Moose." (Rapport Géologique pour 1870-71, page 343.)

"D'une manière générale, on peut dire que toute la contrée examinée, au nord de la région montueuse qui environue le lac ĩupérieur et à l'est du lac Nipigon, est comparativement unie, que le sol est, sablonneux, généralement sec, accidenté en certains endroits par des marais peu profonds et des bancs de roo de peu d'élevation. 
Io vieux bois consiste, par ordre d'abondanoe, en éplnetto blanche, sapin, épinette rouge, bouleau, tremble, cèdre blanc, pin de Banks ou " cyprès " et peupierer balsamique; mais dans lés endroits ravagés par le fer, la nouvelle forêt se composio principalement de boulean et do tremble. L'épinette ronge, le pin do Banks el do cèdre blanc auront une grande utilité pour faire des traverses de chemin de fer et des poteaux cle télégraphe, lors de la construction projetée du chemin de fer Canadien du Pacifique, en mème temps que la région travorsée par n'importe quel tracé ou adoptera pour ce chemin, fournira tonjours une aboudance de bois de chauffage, " (Rapport Geologique pour 1870-72, page 350.)

"Le bois, le long de la partie iniérieure de la rivière Nelson, consiste principalement en épinettes blancho et rouge, tremblo et baumo de Giléad. Sur les lles et les terrains bas, l'épinette blanche atteint une assez boune grosseur et serait très propre à la construction; mais sur le terrain élevé qui s'étend au loin au niveau des berges, le bois est plus petit et le sol est couvert d'une épaisse coacho de mousse, sous laquelle un lit de tourbe d'épaisseur variable se montre sur le bord de chaque banc d'argile escarpé." (Rapport Géologique pour $1877-78$, page $13 \mathrm{cc}$.

" Une petite étendue de bois a été préservée du feu sur le côté oocidental de l'ile Ross, où la rivière de l'Ouest entro dans le lac des Grands-Roseaux, et dans cet endroit beaucoup d'épinettes blanches mesurent trois pieds de diamètre. Les terrains, même les plus ro. cheux, supportent une forêt d'arbres assez gros pour pouvoir servir a plusieurs usages, si ce grond territoire vient un jour à être habité par uno population civilisée. (Rapport Geologique pour 1877-78, page 29cc.)

"Cette région (à l'onest du fort du lac Long) est reconverte d'un sable jaunatre fin au dessous duquel on trouve en quelques endroits un épaisse couche de gravier, à certaines places, une glaise de couleur légère... Le bois se compose de bouleau, de tremble, d'ópinettes rouges, de sapius, de cèdres blancs et de pins de Bank ou cyprès ; beaucoup de ces arbres sont assez gros pour avoir de la valeur comme bois de charpente...On dit qu'une contrée semblable s'étend dans le voisinage de la hautenr des terres, à l'ouest jusqu'au lac Népigon et à l'est jusqu'au fort New-Brunswick, sur la rivière Moose "(Idem, page 343.)

Région de la rivière Moose- "J'ai pris arec soin des notes sur labois qui se rencontre dans la région quo j’ai parcourue ; mais ces détails ne seraient pas à leur place dans le présent rapport. Cependant, je puis mentiouner le fait que les bois de la plus grande valeur, c'est- $\mathbf{-}-$ dire le pin blauc et le pin rouge, sont communs partout entre le lac Yenson et le lac Kenogami, et qu'on cesse de les trouver un peu plus bas que le lac Kenogamisi. En venant du Fort Moose a Michicopoten, j'ai remarqué ces bois pour la première fois au lac Missinaibi, et je ne les ai rencuntrés que rarement entre ce lac et le lac Supérieur. (Dr Bell, Rapport Geologique pour 1876-76, page 841.) 
"Lo pin blane et le pin rouge se rencontrent dans toute la région explorée et sont ioin d'être rares même aussi au nord que le lac Abittibi ; mais sur les bords de ce lac, à l'exception de quelques arbres vigoureux, d'environ six pieds de circonférence, dans les voisinage de la décharge du lac, ces pins sont tcins très petits et nouenx, et ue croissent que sur les nombreuses lles et sur les pointes. Ils sont tout à fait abondants et d'excellente qualité sur ies versants des collines des deux côtés de la hauteur des terres. En faisant l'ascension de la colline décrite comme s'élevant à une hauteur de 700 pieds au-dessus du lac Matawagogig, sur le côté nord de la hanteur des terrc, j'ai mesuré plusieurs beaux arbies et trouré qu'ils avaient de huit à neuf pieds de circonférence à quatre ou cinq pieds du sol, et du sommet de la colline, j'ai aperçu des bosquets de pins blancs dans toutes les directions...L'espèce la plus abondante dans cette région, an nord de la zone de l'érable à sucre, c'est le tremble, puis le bouleau à canot, l'épinette blanche, le cyprès et le sapin." L'orme et le frêne se trouvent quelquefois sur les terrains bas et plats jusqu'au nord du lac Abittibi."( MicOuat. I upport Geologique pour 1872-73, pages 132 ct 133.)

"Depuis la hauteur des terres jusqu'au lac Abatagomaw (entreles sourees du Saint-Maurice et le lac Mistassini), distance de cinq milles, le terrain est un peu plus inégal, mais encore sablonneux et aride, généralement couvert de petits cyprès, avec un pen d'épinette blanche et de bouleau blanc... Le lac est parsemé d'tles rocheuses et basses, s'élevant rarement à plus de trente pieds an-dessus de la surface de l'eau. Le bois, cependant, est plus gros sur les lles et sur les bords du lac.

"Sur le côté snd-est (du lac Wakintche), une grande ótendue đa terrain a été dévastée par le feu et le reste esi parsemé de bois verts ; les arbres sont de bonnes dimensions et appartiennent aux espèces ordinaires-épinette blanche, bouleau blanc, épinette rouge, avec un peu de sapin. Do ce côté, aussi loin que j'ai pu apercevoir, l'élévation du terrain au-dessus du lac est de 100 à 150 pieds et le sol se compose d'une terre jaune sablonneus', bien propre à l'agriculture."

(Richardson. Rapport Geologique pour 1870-71, pages 301 et 302.)

East-Main.- "Le long du rivage oriental de la baie James, depuis le voisinage du fort Rupert jusqu'au cap Jones (distance de 350 milles), il y a une lisière de terrain, ayant une largeur moyenne de pent-étre vingt à trante milles a partir du rivage de la mer, qui, d'après ce que j'ai appris des autres et observé moi-même, paraft devoir avoir un jour à venir rne certaine valeur agricole... Ce terrain est boisé d'épinettes blanches, d'épinettes rouges, de peupliers et de petits bouleaux blancs. Au fort (reorges j'ai vu une quantité de bous billots d'épinette blanche qui araient été descendus sur la GrandoRivière pour servir à la construction des batisses. Beaucoup de ces hillots mesuraient deux pieds de diamètre au bout, et l'âge moyeu des arbres était de près de cent ans. " (Dr Bell-Rapport Géologique pour $1877-78$, page $24 \mathrm{c}$.)

Par tous ces renseignements, il cat facile de constater que dans 
toutes les parties de la région que nous étudions, les forêts ont une valéur indiscutable et renferment des essences qui sont assez développées et en assez grande quantité pour faire l'objet d'une exploitation avantageuse, quand le pays sera ouvert à l'agriculture et au com. merce. Dans les régions susceptibles d'être habitées et cultivées avec profit, dans la vallée supérieure de la rivière Nelson, dans toute l'immense contrée qu'égonttent les rivières Moose et Albany ainsi que leurs nombreux attluents, même dans la région de l'East-Main, l'épinette blanche forme des forêts d'une grande étendue et dont les arbres ont en moyenue de dix-hnit à vingt-quatre pouces de diamètre. l'épinette blanche n'est pas plus grosse dans la provinse de Québec, où elle est si recherchée depuis quelques années et où elle est l'objet d'un commerce si considérable. Quant aux forèts de jins blancs et de pius ronges formant une lisière qui s'étend depuis lés environs du lac Abittibi jusqu'aux suurces de la rivière Albany, bien au-delà da lac long, elles out une importance et une valeur énormes, qui ne feront que s'accroitre avec le temps. Enfin, les forêts de sapins, de peupliers, de trembles et de bouleaux out bien leur utilité: les arbres qu'elles renferment sont assez gros pour faire du bois de chauffage, des perches-ce sont les perches dont on se sert daus le Nord-Ouest pour cloturer-et servir à tous les usages clomestiques, sans compter que plus tard ces esserces pourraient être utilisées aux fins de l'industrie, notamment pour faire de la pulpe de bois, ainsi que cela se pratique dans le nord de l'Allemagne et ailleurs.

Par la description que nous avons donnée du cours des principales rivières, il est facile de roir qu'il n'est un pays qui offre plus do facilités pour la descente des bois que la région de la baie James. Ces rivières forment les grandes artères du systèrne hydrographique de cette contrée ; mais elles ont nu nombre infini d'affuents plus considérables, qui sillounent le pays en tous seus et permettraient d'aller chercher le bois dans toutes les parties de cette irnmense région. Les chutes considérables, qui nécessiteraient des travaux un peu dispendieux pour rendre ces grandes rivieres flottables tout le long de leur's cours, sont comparativement très peu nombreuses. A partir de la chute Couchichnig, d'me cinquantaine de pieds de hauteur et à sept milles du lac ou elle prend ses eaux, la rivière Abittibi n'a qu'une déclivité de 757 pieds sur un parcours de 207 milles, ce qui ne fait pas quatre ponces au mille. Elle forme une vingtaine de rapides, qui penvent presque tons être descendus et remontés en canot, ce qui pronve que cette rivière est parfaitement flottable. La rivière Mattagami semble offrir le même avantage. Sur nne distance de 116 milles, elle n'a qu'une pente de 430 pieds, et le reste de son conrs. 82 milles, est uniforne, sans rapides ni cascades. Le cours de la Missinaibi est d'environ 220 milles, et libre de toute obstruetion jusqu'au pied du Loug-Portage, à 85 milles do son confluent et à 136 milles de la baie Janes. Les quatre-vingt-dix milles, à partir du Long-Portage et en remontant. sont accidentés par une ringtaine de rapides dont l'élération collective est d'a peu près 520 pieds. Sur tout ce parcours, 
il D'y a que denx petites chutes : une de dix pieds et nne autro de vingt. La rivière Kenogami, longue de 214 milles, en y comprenant le lac où elle a sa source, n'a, dans tout ce parcours, qu'une pente de 285 pieds. Il y a près de 158 milles d'eau tranquille : 68 milles de lac et 90 milles de rivière, depuis l'lle Pembina jusqu'aux Fourches. La branche principale de la rivière Albany est navigable jusqn'd Martin's Falls, ¿ 250 milles de son embouchure. Plus haut que la chute Kagiami, qui a 45 pieds de hauteur, le cours de la rivière n'est intercepté que par de petits rapides jusqu'à 65 milles plus loin, a la tête du lac Ábagotikitch wan, qui se trouve à plus de 300 milles de la mer. Le cours des rivières Hayes et Nelson offre aussi beaucoup de longs intervalles d'eau tranquille, et la flottaison du bois pourrait se faire sans beaucoup de difficulté dans ces rivières ainsi quo dans celles de l'East-Main. On peut donc dire, sans crainte d'être démenti par l'expérience, que dans ces régions l'industrie forestière a certainement un avenir précieux. Il y a énormémént de bon bois de commerce et de grandes rivières "qui fourniront d'excellentes voies de transport pour l'amener à la mer.

\section{OHAPITRE VI}

\section{EQQUISBE MINERALOGIQUE-ESPLCES MINKRALES SUSCEPTIBLES}

\section{D'EXPLOITATION ET LEUR EMPLOI}

On a trouvé dans le territoire de la baie d'Hudson des échantillons de presque toutes les espèces minérales qui se rencontrent au Canada. Les explorations qui out été faìes jusqu'à ce jour ne sont quo très incomplètes; on comprend que dans une région aussi vaste, où il n'y a pas actuellement d'autre moyen de trausport que le canot d'écorce, où la forêt recouvre partont le sol, il est très difficile de faire une étude détaillée des roches et jninéraux qu'elles contiennent, on peut tout au plus se former des conjectures basées sur des faits généraux, observés en certaines localités. Cependant les membres de la com. mission géologique du Canada, notamment M. le Dr. Bell, ont réussi à constater l'existence, en quantités inépuisables, de minerais susceptibles d'exploitation. Les recherches laborieuses de ces hardis éclaireurs de la science géologique en Amérique ont amené à notre connaissance des gisemeints de fer qui seront arant longtemps l'objet d'un exploitation aussi lncrative qu'avantageuse au pays. Il y a là du fer, du plomb, du lignite, du manganèse en quantité susceptible d'exploitatation, et 'on a trouvé en plusieurs endroits du gypse, du pétrole, du zinc, de l'argent, des traces d'or, du molybdène, de l'asbeste, du cuivre et plusieurs espèces de pierres d'ornementation qui pourraient bien former des gisements d'une grande valeur. Il $\mathrm{y}$ a aussi plusieurs variétés de serpentino et de la bonne tourbe en abondance. 
Tous les voyagenrs et les explorateurs qui ont écrit sur la région de la baie d'Hudson depnis un siècle et demi ont constaté l'existence de ces richesses minérales. "Pour ce qui est des minéraux, écrivait Ellis il y a plus de vingt-cinq ans, il y en a sans contredit, beaucoup de différentes espèces. J'ai trouvé moi-même des mines do fer, et j'ai appris de boune part qu'on trouve à Churchill des mines de plomb a fleur de terre, sans parler d'une riche mine de cuirre, dont les Indiens septentrionaux apportent souvent de grands morceaux à Churchill; j'en conserve moi-mène encoro un. Il y a aussi de différents talcs en abondauce, des verres de Moscovie. On trouve aussi dans les parties septentrionales une matière combustible semblable à du charbon. L'Asbestos semblable an lin de roche (stone glass) est encore fort commun dans ces endroits, de même qu'une pierre d'uue surface noire, polic et luisante, qui se sépare parfaitement en feuilles transparentes, Eemblable au talc de Moscovie et dont les originaires se servent au lieu de miroirs. On y trouve aussi en abondance diverses espèces de marbre dont quelques-uns sont parfaitement blanes et d'autres djfféremment coloriés de rouge, de vert et de bleu." (Ellis' voyage vo N.-B., traduetion trançaise, page 219.)

Robson, qui a passé six ans a la baie d'Hudson, de 1733 à 1736 puis de $1744 \mathrm{a} 1747$, dit à pau près la mêmo chose dans son livre intitulé : Account of six years residence in Hudson's Bay. " Outre, dit-il, les pêcheries et le commerce des palleteries, et le fait que ces industries sont susceptibles d'un développement incontestable, il $\mathrm{y}$ a les plus fortes apparences de riches mines dans les différntes parties du pays. J'ai vu des morceaux de minérai brillant qui avaient été apportés de la colline de Knight,... et il a été établi dans l'euquête faite par le comité (de la Chambre des Communes en 1749) qu'on avait apporté aux comptoirs du sud du minérai dont on faisait des boncles; qu'il y a sur les bords de l'East-Main des mines de plomb très précieuses et dont on a produit des échantillons de minerai, et qu'il a été trouvé sur la côte, entro les rivières Churchill et Nelson, du cinabre natif dont on a extrait du mercure qui fut envoyé comıne échantillou à la compagnie. Il y a aussi la plus grande probabilité qu'il existo une riche mine de cuivre au nord-est de la rivière Churchill, j'ai va plusieurs morceanx de ce minérai ; les sauvages de cette région les portent comme ornements aux oreilles et aux poignets, et un monsicur qui était présent au règlement de comptes, à la rivière Churchill, m'informa que les sauvages ont des ciseaux à glace et d'autres outils faits avec ce cuivre." (page 69.)

L'exactitude de la plupart des renseignements donués par ces deux écrivains est corroborée par le Dr Bell, l'un des nembres les plus citpables et les pius distingués de la Commission Géologique du Canada. Cette unanimité de témoignages relatifs à la richesse minérale de la baie d'Hudson établit clairement qu'il y a là des gisements métalliforres d'une importance incontestable. Les rapports de la Commission Géologique nous donnent les renseignements qui suivent sur 
les espèces minérales trouvées jusçu'aujourd'hui dans la région do la baie d'Hudson.

Plâtre-Après avoir passé les Grands-Rapidès, je n'ai pas vu de roches in situ avant d'arriver au Buncs Blancs, ou do plâtre, sur le cours principal de la rivière Moose. Ces bancs so voient sur les deux bords de la rivière et commencent à trente-hnit milles plus haut que le fort Moose. Le banc du côté sud-est' s'étend sur nne distance d'environ deux milles, et celui du cóté opposé sur environ la moitié de cette distance. Il forme une couche, appartenant à la variété commune, hydratée greune, se prolongecnt des deux côtés de la rivière et ne s'élevant pas à plus de dix pieds an-dessus du nireau des basses caux. Ce gypse est généralement de couleur gris-blenâtre, arec quelques parties blanchâtres tachetées de jaune et d'autres couleurs. Je n'ai pas vu la variété blanche, dont on se sert 'pour faire le strie, en assez grande quantité pour avoir une raleur industrielle. Cette couche est recourerte des deux côtés de la rivière par un lit mêlé de gypse et de marne gris-bleudtre, ayant aussi lui dix pieds d'épaisseur. 'Lo gypse est par masses, dont beaucoup se composent de sélénite transparente, incolore, clivable en lames minces. Un banc de gypse, semblable à celui qui vient d'ètre décrit, se trouve sur le côte sud-est de la rivière, à quatre ou cinq milles plus bas que l'extrémité de l'autre banc semblable qui se trouve du snême côté." (Dr Bell - Rapport Geologique pour 1875-76, page 321.)

Le Dr Bigsley prétend que ee gypse est presque caracteristique des calcaires de la baie d'Hndson. Si cette opinion est bien fondée, on peut s'attendre à le trouver eu beaucoup d'autres endroits. Cependant les gisements signalés par le Dr Bell sont assez considérables pour ètre exploités. Co gypse n'est ni assez blauc ni assez fin pour servir à la préparation du strie ou plâtre de Pasis ; mais il pent être employé dans l'agriculture, qui en consomme d'énormes quantités. C'est un des meilleurs fertilisants et l'un des moins dispendienx. Lue fait que 'la rivière Moose est narigable jusqu'à l'eudroit et même plus loin où on les tronve sur ces bords, semble assurer à l'exploitation de ces dépôts de plâtre un brillant avenir.

Pierre de savon - "Les Esr vimaux de la cóto orientalo et des íles de la baie d'Hudson emploient une pierre de savon grise pour faire leurs chaudieres et leurs lampes, qui ont souvent plus de cieux pieds de longueur. Cette pierre appartient à une rariété résistable et durable. Je les ai vus boncher des trous formés dans plusieurs de leura chaudières par un long usage, en y insérant de nouveaux morccaux de pierre cimentés avee de la terre glaise. Autant que j'ai pu le saroir d'eux, cette pierre de saron se troure à peu de distance de la baie des Mariugonins, par $60^{\circ}$.5 de latitude." (Dr Beil-Rapport Géologique pour 1877.78 , pages 23 et $24 \mathrm{c}$.)

La pierre do saron, ou stéarito, est un talc plus on moins pur. Elle est assez tendre pour se tailler de toutes les formes voulues arec des couteaux ou des scies. Flle est infusible à la température ordinaire des fourneaux. Quand elle est pure et compacte, on s'en sert comme 
matière réfractaire pour ondurcir l'intérieur des fournaises et pour faire de petites fournaises portatives. On l'emploie pour fabriquer des rases de cuisine et des tuyaux pour l'cau, surtout pour faire des rases déstinés à contenir des acides et des alcalis. Soumise à une forte chaleur, la pierre de saron perd l'eau qu'elle contient et prend ensuite un bean joli et se colorie facilement. Ainsi préparée, on l'emploie pour faire des boutons ot autres petits artieles de brinbloteni. On en fait aussi des bees de gaz, qui ont sur les autres l'avantage de ne pas se brûler ni se corroder. Pulvérisée, la stéatite s'em. ploie pour lubréfier les machines et donncr le poli à certaines espèces de papiers de tentures. Réduite on poudre et teinte par des acides, la stéatite fait une bonue peinture commune, qui est beancoup en usage aux Etats-Unis. Enfin, c'est avec la stéatite on pierre de saron qu'on fabrique les crayous dont les tailleurs se servent pour carter ainsi que pour faire la craie rénitienne, qui entre daus la composition de certaines couleurs.

Pierres ornementales-Parmi les pierres susceptibles de poli et d'emploi comme ornement, on pent mentionner les agates, les carnolines, Jes épidotes et Jes porphyres des roches de trapp qui se trouvent ontre les détroits de Manitounuck et de Nastapoka. Les agates sont très abondantes, et souvent d'une bonne grossenr, dans ces arnygdaloides ; mais elles sont généralement grossières, mal colorées, et après les avoir iart essayer par plusieurs lapidaires, on a trouvé qu'il est difficile de leur douner un poli bien fin. La caleédoine rouge que l'ou trouve en lits sur l'tle Davian et dans plusieurs autres lles de la chatne Natas. kopa, et la calcédoine vert-olive, ressemblent au jade, quo l'on a trouvée en petiter reines dans l'sle Búlanger, pourant être polies comme pierre ornementales. L'asinerite, ce rure minéral qu'au Canada on n'avait pas encore trouvé $z$ situ, a été vu en petites reines dans le trayp, sur la côte, environ à un mille et demi au sud de l'embouchnre de la petite rivière à la Baleine, en crystanx, avec du quartz, du spath calcaire, de la chlorite et de l'asbeste, et aussi sous la forme granulaire. Les deux rariétés sont rouge-pourpre, et la variété granulaire prend un poli très fin. Le jaspe ronge, avee des particules flottantes, comme celui de la série Népigon, prèrs de la baie du Tonnerre, sur le lac Supérieur, se tronve sur l'sle Sungue. Une argilite vert-grisâtre avec des bigravures noires, cormme celle que les Śauvages du Camada employaient autrefois pour confectionner des articles de parure et autres, a été trourée dans une falaise sur le cồé nord de la petite rivière aux Baleines. Les crystaux de quartz transparent, qui abondent dans les druses de la bande plombifère de la petite riviere aux $\mathrm{Ba}$ leines, \&s., peuvent êtro mentionnés comme se rattachaut à cette argilite. On rapporte qu' ne piorre verte molle, comme la serpentine, que les sauvages taillent pour faire des pipes, se trouve à quelques milles au nord du fort George, mais je rn'vi pas été capable de trouver cet endroit. Dans le voisinage, la rochs est un gniers laurentien." ( Di. Bell-Rapport Géloginue prour 1877-78, pages 220 et 23e.)

Ces pierres ont beaucoup de raleur dans la joaillerie, et d'après la 
description qui précède, il est évident que plusieures pourraient faire l'objet d'une exploitation lucrative.

Pyriles "A plusieurs endroits, le loug de la côte, j'ai mentionné des bois de pyrite de fer en parlant de la présence de l'or et de l'argent. Je l'ai aussi trouvée en grappes dans lest arnygdaloïdes dans les envirnns du golfe Richmond. En 1875, des échantillons de pyrite massive et d'autres variétés de pyrites de fer provenant du voisinage de la petite rivière aux Baleines m'ont été présentés. " (Idem, page 22 c.)

La pyrite ne vaut rien comme minerai de fer, mais elle est beaucoup employée dans la préparatior de la comperose et de l'acide sulfuri. que. Il s'en importe annuellement plus de trois cent mille tonneaux en Angleterre, où elle vaut une dizaine de piastres le tonnean. L'acide sulfurique que l'on tire de la pyrite de fer sert a la fabrication du carbonate de soude et de la soude caustique, a la préparation du phosphate de chanz, de l'alun, de l'acide nitrique et à la clarification du pétrole. Seulement dans le Lancashire, en Angleterre, on emploie plus de quatre tonneaux d'acide sulfurique par semaine à ces différents $\mathrm{u}$ :

Manganèse- "Les minerais de fer spatique décrits plus haut sont si richez en manganèse, que l'on a raison de s'attendre à trouver l'oxyde noir de méta? dans quelques parties des endroits où se trouvent ces minerais. Comme je l'ai déjà dit, la forte proportion de manganèse que contiennent ces minerais les rendra précieux pour la tabrication du Sprigeleisen ou fonte blanche miroitante, et à raisor de leur abondance et de leur accès facile, on trouvera peut-être un jour profitable de les transporter en Angleterre et aux Etats-Unis. " (Idem, page 22c.)

Le manganèse est presque toujours associé aux minéraux de fer spatique, qui en contiennent ordinairement jusqu'à 10 pour 100 . Les mines de fer spatique de l'lle Flint, près du rivage oriental de la baie d'Hudson, renferment au delà de 24 pour $10 \mathrm{C}$ de carbonate de manganèse.

C'est avec le chlore, indirectement avec l'oxyde de manganèse qui sert à le préparer, que l'on décolore et blanchit promptement les fils et les tissus de lin et de coton, la cire et les autres objets susceptibles de supporter l'action de ces agents chimiques. On emploie aussi le manganèse dans la teinture et l'impression des calicots, dans la verrerie et la poterie. Un peu de manganèso oxydé, jeto à propos dans lo creuset du verrier, fait disparattre les nébularités noirâtres causées par la présence de molécules charbonneuses dans la masse en fusion ; Ia plus grande partie du manganèse employé dans les manufactures anglaises est importé d'Allemagne, et cette importition excòde chaque année 30,000 tonneaux.

Zinc - " La blinde (suifate de zinc) se troure avec le spath calcaire en petites veines coupant les dolomites, sar le côté sud de la petito rivière aux Baleines, et en crystaux associés à des grappes de galène dans la bande plombifère des autres localités. Quoique je ne l'aie trouvé nulle part en quantité susceptible d'exploitation, la présence 
de ce minerai mérite d'être signalée, vu qu'on pourra peut-être deccuvrir qu'il existe en plus grande abondance dans cette partie de la côte. "' (Dr Bell-Rapport Géologique pour 1877-78, page 21c.)

Quoique moins abondante, dans ses gites, que le zinc oxydé, la blende est aussi commune et aussi répandne que ee minerai. Il est bien rare qu'elle n'accompagne pas le plomb' sulfuré et mème quelques autres substances, telles que l'argent sulfuré, le cuivro pyriteux, le cuivre gris, le fer sulfuré,le fer carbonaté,ete. Comıne plusieurs de ces minerais se rencontrent dans le voisinage de la localité où l'on a tronvé ces petits gisements de blende, M. le Dr Bell a raison d'espérer qu'ici comme ailleur's on finira par la trouver t!l quantité suffisante pour la rendre susceptible d'exploitation. L'exploitation des mines de sulfure de zinc n'est orünairement que l'accessoire de celles des mines de galène ; elle pourra se faire pareillement à la riviöre aux Baleines, qui n'est pas éloignée de bonnes mines de plomb.

Plomb-" An fort Moose', les employés de la compagnie de la baie d'Hudson m'ont donné des échantillons de pyrites de fer massires, de chert couleur de fumée foncée, comme celui de la baie du Tounerre, d'épidosite, d'agate, de eornaline, de crystaux de quartz, de galène, de sidérite crystalline noire contenant une forte quantité de manganèse : tous ces échantillons avaient été pris dans les environs de la petite rivière aux Baleines. La galène provient d'une veine, sitnée dans les terres à quelques milles de l'embouchure de la rivière, qui fut jadis exploitée par la compagnie de la baie d'Hudson. D'après l'analyse du Dr Harrington, ces éehantillons de galène contiennent 5. 104 onces d'argent par tonneau de minerai. Cette veine est dans une gangue de spath calcaire et la roche enviromnante paratt ètre un calcaire compact, à crystaux fins, d'une conleur gris-rongeatre foncée." (Dr Bell-Rapport Gsologique nour 1875-76, pages 323-324.)

" La baude plombifère parait être fréquemment exposée dans les escarpements de la côte entre le détroit de Manitounuck et le golfe Richmond et sur la rive ouost de ee golie. Qnoique comparativement mince, cette bande est probablement continue dins cet intervalle et à raison de sa richesse en galène, elle pourrait avoir de l'importance an point de vue économique." (Dr.Bell, Rapuort Géologique pour 187ケ.78, page. $15 \mathrm{c}$.

"Dans la partie inférieure de la conche de calcaire magnésien des séries, il y a une bande d'environ trente pieds d'épaisseur... dans laquelle la galène, en grappes, se troure en quantité suffisante pour ètre exploitée. En 1858-59, la compagnie de la baie d'Hudson a fait extraire neuf tomneaux de ce minerai de nombreuses petites ouvertures qui furent pratiquées à environ trois milles au nord-est de son établissement de la petite rivière anx Baleines; mais le minerai parait étre érgalement aboudant ou plus abondant dans quelques endroits do la bande de caleaire du côté sud de la rivière. Cette bande se continue jusqu'au golfe Richmond, à l'entrée duquel j'ai trouré dans la mème bande des grappes de galène qui peseraient au delà de cent livres. Le Dr Harrington a trouvé que des échantillous de la 8 
"mine" du côté nord do la petite rivièro aux Baleines contennit 5.104 oncos d'argent par tomeau de minerai. Il a tronvé que lo minerai pris sur lo côté sud de l'entrée du golfo de Richmond contient, sépalé de la gangue. 12.03 onces d'argent par tonneau de minerai de 2000 livres." (1. Boll-Riuport Géologique your 1877-78, page 20c.)

Quand il est par, co minerai contient 86.6 de plomb et 13.1 de soufre. Mais il renferme presque toujours me certaine partio d'argent, ce qui lni donne la composition suivante: plomb, 84 ; soufre et autres matières, 14 .

Il n'est guère besoin d'insister sur l'importance de ces mines de plomb, qui se trouvent on ne pent mieux situées pour être exploitées arec la plus grande facilité. Combien de millions de livres de plomb ne pent-on pas tirer de cette bande de galène qui a vingt pieds d'épaisseur où elleøa été examinée et peut-être plus aillenrs, et une longueur d'une vingtaine de milles ! Et si, comme on l'a constaté, cette galène contient de einq à treize onces d'argent par tonnean de minerai, il est facile de voir que ces immenses dépôts plombifères renferment réellement des richesses considérables. En Enrope; la quantité d'argent que contient la galène est g'énéraloment assez forte pour dédommager des frais d'alfinage des minerais et procurer mème des bénéfices.

Molybuène-" A la grande rivière anx Baleines, on m'a douné un échantillon de sulfure de molybdène qu'on n'a dit avoir été trouvé dans les environs." (Idem, page 22c.)

Le molybdène sulfuré ne se tronvo ordinairenent que dans les terraius primitifs, où il ne forme ni couches ni filons, mais senlerisent des petits dépôts ou rognons, dans une gangue de schiste micacé, généralement avec du cuivre gris et du cuivre pyriteux. Ce mineral a une très grande valeur ì cause de sa rareté. Sir William Logan dit $q u$ 'à l'exposition de Londres, en $1 \times 62$, il était coté à \$3.45 li livre, à l'état brut. Onl'emploie dans la chimie comme réactif et dans la teinturerie pour domner ce qu'on appelle les couleurs topiqnes, et surtont pour préparer lo bleu Thénard, qui résiste parfaitement à l'action de l'eau et du soleil.

Cuivre.-"J'ai déjà mentionné le fait que l'on tronve des pyrites cuirreuses dans lo roisinage de la montagne à la Peinture, sur les bords du lac Abatagomaw (près du lac Mistassini). A un endroit situé un peu au sud-onest de la montagne, sur la rive du lac, il y a des indices de ce mincrai ainsi que des taches de carbonate de cuivre vert ; mais je n'ai pas vu de couches on de veines bien définies IAa roche est un schiste chloritique vert, nu peu calcaire. Cesindices de cuivre se voient sur une distance de pris d'un demi-mille en allant au nord-est, le long de la rive du lac, jusqu'à un autre endroit où uno couche ou une veine de deux pieds d'épaisseur, contenant des pyrites de cuivre, se trouve dans la roche chloritique sur une étendue de vingt pieds. La partie de la veine qui est exposée dcnnerait probablement partont quatre ou cing pour cent de cuivre; et certaines parties pourraient donner de dix à douze pour cent. Sur une dis- 
tance additionnelle d'environ trois quarts de mille, lo long du lac, partont où la roche parait, on voit dọs indices de sulfure janne et de carbonate vert de, cuivre. An bout de cette distance et immédiatement au bas de la montagne à la l'einture, la roche est fortement chargée de pyrites de fer à grains fins et de taches do sulfure jaune, dans une gangne quartzense jaune. Ici les pyrites de fer forment au moins quinzo on vingt pour cent de la roche, et sur toute la distance déerite plus hatut, environ un mille et quart, on la roit partout, mais en petites quantités. A l'endroit mentionné en dernier lieu, il y a la dépression mentionnée à la page 243. Comme je l'ai dit déjà, cette dépression est remplie do matières de transport et la roche ne s'y voit pas ; mais à en juger par les quantités de pyrites de fer et do cuivre qu'on tronve dais cette roche des deux côtés de l'eufoncement, il est possible qu'il existe un précieux dépôt de minerai de cuivre sous ces matières rupportées. "(Richardson-Rapport Geologique pour 1870-72, pages 295 et 296.$)$

"Dans les onze milles qui suivent (66 milles plus bas que le confluent de la rivière Muskootasagaigan, sur la Mattagami) des diorites molles, de couleur gris-verdâtre un pen léger, massives, esquilleuses, se voient à plusieurs endroits. I'espèce esquilleuse est truversée à une place par des reines de spath calcaire de trois ì dix pouces d'épaissenr, renfermant des indices de pyrite cuivreuse. "(Dr Bell-Rapport Géologique pour 1875- 76 , page 312.)

"On m'a présenté quelques échantillons de pyrite cuirreuse pure, associéss avec du spath calcaire et dess crystaux de quartz, qu'on dit avoir été ramassés dans les environs du golfe Richmond, et j'ai tronvée des taches du même minerai dans des petites veines de spath calcaire environ un mille an nord de l'entrée de ce golfe. Sur l'ile Longue, du côté de la terro ferrne, à environ trois milles de son extrémité sud-onest, de nombrenses veines de spath calcaire et d'autres minéranx qui coupent le trapp, contieunent des taches et de petites grappes de pyrite cuivreuse. "(Do, Rapport Géologique pour 1877-78, page 20c.)

La pyrite cuivreuse, on euivre pyriteux, qui est d'une extrème importance par la richesse de ses produits, existe dans le sein de la terre en reines, filons et couches, et parait appartenir exclusivement aux formations primitives. C'est in cuivre pyritenx qu'on obtient presque tout le unétal qui alimente le commerce. On en distingue trois es. pèces : le cuivre panaché, ou cuivre vitrenx, qui dans son état le plus pur contient 78.8 de suivre et 10.2 de sonfro ; l'érubescile on cuivre pouryre, qui renferme du fer et du sonfre et donne de 56 à 62 pour 100 de cuivre, lorsqu'il est très pur' ; la chalcopyrile, ou pyrite de chivre janne, double sulfure de cuirre et de fer, dont les variétés les plus pures produisent à la fusion de 32 à 34 pour 100 de métal.

Les cépôts indiqués par M. le Dr Bell sont plutôt des indices do gisements considérables que des mines d'une valeur appréciable; inais les couches décourertes par.M. Richardson sur les bords du lac Abatagomaw ont une importance réelle. Jeur étendue et la qualité comme la quintité du minerai qu'elles contiennent les rendent sus- 
ceptibles d'uno exploitation aussi facile que profitable. Si le lac Abatagonaw était accessible par quelque grande voio de communication, il y aurait là pour l'industrie minière une riche jnoisson à récolter.

Asbeste-" Trouvée en petite quantité avee l'axinite, déjà mentionnée, ha environ un mille et demi ua sud de in petite rivière aux Baleines. On dit qu'il se tronve en plus grande quantité ailleurs, sur les bords de la rivière, mais on n'a pas constaté où sont ces localités." (Idem, page 23c.)

Ce minéral est employé pour une foule d'usages et se vend comparativement cher. Dans la province de Québec, il se trouve en abondance aux mines de Colraine et de Thetford, d'où on en exporte de grandes quantités aux Ltats-Unis. Comme on le trouve généralement dans les terrains primitifs, dont il ne fait cependant pas partie intégrante, et accompagné par les minerais de plomb, il y a tout lieu do croire que les gisements indiqués plus haut sont réellement considérables, puisque les terrains primitif's et la galène se rencontrent dans les envirous de la petite rivière aux Baleines, où l'on a trouvé cet asbeste.

Pétrole.- "Sur les bords de la rivière Abittibi, on a trouvé des calcaires bitumineux et des schistes carbonifères, appartenant aux formations devoniennes, qui ont une forte ressemblance avec les couches pétrolifères du même âge de la vallée de l'Athabaska-Machenzie. Ces roches se voient tout le long de la rivière Abittibi, entre le vingtneuvième et le trente-neuvième milles, it partir de son embouchure, et à un endroit le ealcaire coutient du pútrole liquide." (Idem, page 24c.)

Anthracite.- "Pendant que j'étais au fort Moose, le capitaine Tay. lor, employé par la compagnie de la baie d'Hudson, m'a donné des échantillons d'un minéral ayant tontes les qualités d'un bel anthracite, excopté qu'il ne contient, d'après l'analyse du professeur Hoffman. qu'une très petite quantité de cendre. Ces échantillons avaient été apportés par un sauvage de l'lle Longue, an sud de la grande rivière à la Baleine. M. James Colter, de la compagnie de la baie d'Hudson, m'a dit qu'au rapport des sauvages il existe un minéral semblable à quelques milles dans l'intérieur, en s'éloignant de la petite rivière à la Baleine. Je n'ai pu rien constater par moi-même rélativement à son mode d'occurrence, si ce n'est que le sanvage qui avait apporté les échantillons de l'ile Longue m'a dit que lì il y en a en aboudance. Ce minéral paraít provenir de l'altération d'un minerai semblable à l'asbeste, par la perte de presque tout son bitume.'

(Dr Bell-Rapport Géologique pour 1875-76, page 325.)

" L'existence de ce précienx minéral dans l'nle Longue a été mentionnée à la page 25 de mon rapport pour 1875. Il a une fraeture conchoide et un lustre brillant, et M. Hoffinan a trouvé qu'il contient 94.91 pour cent de earbone fixe et seulement 0.35 pour cent de cendre. Il est probable qu'il ne forme pas une veine de charbon bitumineux altéré comme l'unthracite ordinaire, mais qu'il est plutôt le produit 
d'ume poix durcie. ou un minéral comme l'albertite, par la perte de son bitume, et il pent so faire qu'il n'existe pas en grande quantité. J'ai été empèché par les circonstances de visiter la localité où l'ou trouve cet anthracite et qu'on dit être sur l'ile Longue, à quatre ou cinq milles de son extrémité sud-ouest. " (Dr Bell-Rapport Géelogigue pour $187 \%-78$, page $24 \mathrm{c}$.)

"Il n'est pat inprobable que ce minéral ait uno origine semblable à celle de la matière anthracitique noire qui se reacontre en beanconp d'endroits du gronze de Québee. L'échantillon analysé est très com. pact, homogìne ; conleur, noir poix ; poudre, noir foncé ; lustre, inétallique brillant ; frecture, srès conchoïde; ne tache pas les doigts... Les chiffres qui suivent sont le résultat moyen de denx analyses , très concordantes:

Carbone fixe .94 .91

Matière rolatile combustible.......................... 1.29

Ear..................................................... $\$ .45$

Cendre............................................. 0.85

100.00

"La transformation en coke a à peine changé son apparence." (Hoffman-Rapport Géologique pour 187i-78, page 423.)

Que cet anthracite soit plus ou moins parfinit an point de vue rigoureux de la science. il n'en constitue pas moins an bon combustible et une souree de richesse incontestable, s'il existe en grande quantité dans la région où il a été trouvé.

Lignite. - "Juste an bas de l'embouchure de la rivière aux Oies, ou trois milles plus bas que l'endroit où la rivière (Albany) tourne au sud-est, il y a do la marne rouge brillante sur le côté nord, dans une petite lle: un mille plus bas, j'ai trouvé des fragments dispersés d'uu charbon bitumineux brillant.Les employés de la compagnie de la baio d'Hudson disent qu'il n'a jamais été apporté de eharbon dans cette région, et l'on ne pent pas s'attendre à antre chose quand on considère que le transport même des artieles légers et précieux est si coûteux dans ce pays, de sorte que je ne puis pas supposer que ce charbon ait été apporté par la main de l'homine. "(Dr Bell-Rapport Géologique pour $1870-72$, page 112.)

"Au grand Rapide (sur la rivière Moose) la glaise blene contient les premiers coquillages de mer (Tillina et Leda) que j'ai r'a le long de la rivière. L'élération au-dessus de la mor est d'environ 300 pieds. J'ai trouvé sur la rive, au pied des rapides, un petit morceau do lignite ayant une fracture lustrée." (Dr Bell-Rapport Géologique pour 1875-76, page 320.$)$

"Le lignite, qu'on dit apercevoir in situ lorsque l'eau de la rivière est trìs busse à l'embouchure du ruisseau au Charbon, est probablement associé à ces marnes. Des fragments de lignite sont 
dispersés, souvent eu ahondnnce, lo long du lit de la rivière sur toute la distance entro les Fourches et ce ruissean. Il peut se trouver en benucoup d'endroits avec les marnes de conleur légère qui ont probablement la forme de bassins de pen de profondeur, reposant d'une manière non concordante sur les roches palíazolques de la grande région unie située au sud-onest de la brie James... J'ai trouvó des fragments de lignite semblable sur la Mattagani, commo jo l'ai déjà dit, et sur la rivière Albany... M. Hoffman a analysé un des échantillons que j'ai apportés de la riviòro Missinaibi. " (Dr Bell-Rapport Géologique pour 1875-76, page 326.)

Voici cette analyse :

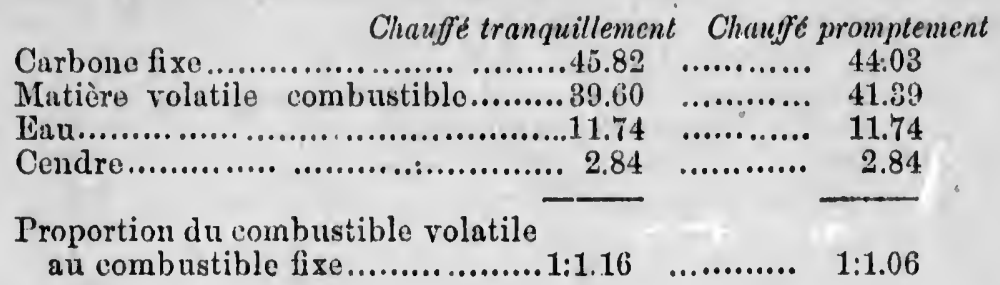

"L'existence du lignite sur ies hords de la rivière Missinaibi a été mentionuée à la page 326 de mon rapport pour 187j. L'été dernier je l'ai vu en plusieurs endroits le long de cetto riviòre entre le LongPortage et lo confluent de la rivière avec la Mattagami. Lo premier ou le plus éloigné de ces endroits est sur la berge occidentale du ruisseau au Charbon, ì trois quarts de mille de son embonchure...Cette couche de lignite a environ trois pieds d'épaisseur et est recouverte nar environ soixante et dix pieds do glaise alluvienne, ou till, mollo ti tenace, pleine de petits gilets et passant au gravier vers le sommet. Une grande partie du lignite a conservé sa texture ligneuse : quelques-uns des troncs englontis dans cette concho mesurent deux pieds de diametre. Ce charbon fait un bon combustible lorsqu'il est sec, mais il contient un peu de pyrite de fer.

"Sur le côté sud-est de la rivière, à neuf milles plus bas que le ruissean au Charbon on deux milles plus haut que l'1le an Pique-Bois, j'ai trouvé une reine horizontale de lignite an milieu d'un bane de till de 125 pieds de hauteur. Cette veine a de $1 \frac{1}{2}$ à $2 \frac{1}{2}$ pieds d'épaisseur et elle se compose en grande partie de branches et de joncs. Au-rlessus du lignite, il y a 80 pieds de glaise grise deveuant jaune lorse al! est exposée à l'air et an-dessus il y a 45 pieds de glaise bleue

"A trois milles plus bas que l'yle an Pique-Bois ou neuf mil plus haut que la rivière Opozatika (Penplier), il y a dans le bord te la rivière, sur le mème côté, une autre couche de lignite. Elle a six pieds d'épaisseur, nais diminue en allant à l'est et elle a la inême texture chisteuse, se composant de lammelles de tiges et de moirsse. Immédiatement au-dessous du lignite, il y a une conche d'un pied d'épaisseur do glaise irrégulièrement mèlée avec des amas do lignite 
impur...Au-dessus du tont et formant le sommet du banc, qui a soixante-cinq pieds do hanteur, il y a dix pieds de glaise dure brane...

"J'ai anssi vu.de petites conches minces do lignite ì deux endroits sur le même bord do la rivière, au pied du rapide qu'il y a six milles plus hant gue la rivière Opayntika, puis à un demi-mille plus bas encore.

"Dans l'intervalle compris entre un et deux milles plus hant que cet afflnent, tout le lit de la riviere parnit reposer sur le lignite. "(Dr Bell-Rupport Geologique pour 1877-78, page 4c)

"Quelques morceiux détachés de lignite ont été trouvés sur la bergé orriautalo de la rivière Abittibi, un pou plus hant que le ruisseau du Gros-Cèdre, à environ vingt-trois milles de son embouchure." (Idem, page 370)

On divise géneralement la honille en trois classes, qui correspondent à l'âge géologique des conches terrestres dans lesquelles se trouve co précienx minérai. L'anthracite, qui est caractérisé par sa grando dureté et par la petite quantité d'hydrogène qu'il dógage, se trouve dans les couches les plus basses ou les plus anciennes des terrains carbonifëres. Vient ensuite le charbon bitunnineux, ou charbon de pierre, qui est noire, d'un lustre plus ou moins vitreux et renferme de dix à soixinnte pour cent de bitume. On l'appelle charbon gras ou charbon maigre, selon que la proportion de bitumo qu'il renferno est plus au moins grande. Le lignite, on bois bitumineux, se trouve dans les couches snpérieures au-dessus du charbon bitumineux. C'est une houille moins perfectionnée que les autres, ce qui explique pourquoi on la trouve généralement duns les terrains d'une formation moins ancienne et à de moindres profonleurs. Les conches de lignite reposent immédiatenent sur l'argile et ne sont séparées du śol que par des amas de sable on des détritus analognes. Il arrive très souvent qu'au lieu de former des conches, les fragments de bois conservant parfaitement leurs formes originaires et leur texture ligneuse, sont simplement dessiminés daus l'argile, le sable ou le graviër. Ces couches de lignite ressemblent à des morceaux irréguliers de trones d'arbres, de branches et de fenilles. L'apparence extérieure et la texture de vette houille varient antant que sa composition chimiqne. La couleur varie du brun léger an noir foncé. Quelques espèces sont très friables ct d'autres très dures. Le lignite renferme jusqu'à 43 pour 100 d'ıan et il en est peu qui u'en renferme pas an moins 20 pour 100. Exposé à une atmosphere sèche, il se désagrège, se pulvérise et perd une grande partie de son humidité, mais ne devient jamais entièrement sec. Osborne domne l'analyse de quatre échantillons do lignite dont la moyenne a été comme suit: carbone, 59.065 ; hydrogène, 5.087 ; oxygène. 24.28 . Isa quantité de cendre résultant de sa combustion varie de 1.50 , pour la meilleure qualité, à 27.2 pour 100 , pour la plus manraise.

En comparant ces chiffres à ceux de l'analyse faite par le professeur Hoffman du lignite trouvé en immense quantité dans la région de la baie James, on arrive nécessairement à la conclusion que ce li- 
guite est de qualité supérieure, ainsi que le dómontre le tableau suivant :

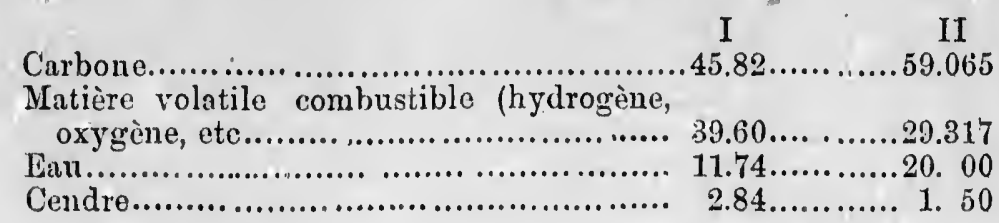

Les chiffres de la colonne I sont ceux de l'analyse faite par le pro. fesceur Hoffman ; ceux de la colonne II donnent le résultat de l'analyse, claprès Osborne, des meilleures espèces de liguite. Il saffit de jetor un coup d'œil sur ce tableau pour vinir que sous le rapport de l'humidité et des matières rolatiles combustibles, notre lignite de la rivière Moose est bien supérieur aux meilleures espèces que l'on trouve ailleurs. Nous pourrions dire la même chose relativement à la quantité de cendre produite par la comburation.

La cendre provenant du lignite renferme généralement beaucoup de matières sulfurenses, ainsi que le uiontrent les analyses suivantes, prises dans Osborne :

I

Sulfate de haux........... 3.6

Sulfite de potasse.......... $\quad 1.9$

Sultite de chanx........... 25.4

Sulfate de fer............... 50.0

Sable

19.1

100.0

II

Sulfate de chaux........... 55.50

Magnésie................... 2.58

Alumine.................. 11.57

Oxyde de fer.............. 5.78

Carbonate de potasse.... $\quad 2.64$

Sable........................ 2.03

100.10

Le lignite, dit Oshorne, est très rarement susceptible d'être employó pour fondre les minerais de fer, un peu à raison de sa friabilité et de son humidité, mais surtont à raison de la composition de ses cendres, Flles abondent généralement en sulfates et en sulfites qui communiquent leur sonflye au métal fondu et le rendent eassant. Il faut donc, ajonte le mème anteur, prendre beancoin de soin pour employer ce charbon dans les forges... Quelques espèces de lignite constitnent nu bon combustible pour les fourneaux à puddler ainsi que pour les fours à réchauffer ; mais leur emploi dans les hauts-fourueaux doit être limité. *

Cé inconvénient, qui se présente pour la fonte des minerais de fer ordinaires, n'exist. pas pour cenx que nous avons dans les mines de Kastakopa. Ce cúinerai renferme une forte quantité de manganèse.

- Usborne, Mutallurgy of Iron and stecl, pages 187 et 207. 
Or il est constaté que la manganèse ábsorbe presque complètement le souffre et le fait passer dans les scories, ce qui fait que pour foudre le minerai manganésifère de Nastakopa, on pourrait suns inconvénient, sous ce rapport du moins, employer le lignite qui se trouve en grande quantité daus la région de la rivière Moose et de la rivière $\Lambda$ libany. Fondu avec la lonte, dit Osborne, le manganèse dégage en grande partie le fer du souffre qu'il contient. Caron affirme que certiins minerais de fer renfermant de la pyrite da cuivre doment un fer libre de soufre, parce que ces minerais renferment aussi beaucoup de manganèse. Pour cela, il suffit de calciner le minerai avant de le fondre. On peut donc affirm $э$ q que les minerais de fer spathique de Nastakopa, qui contiennent juscuu'à 27 pour 100 de manganèse, peuvent être fondus sans inconvénient en employant comme combustible le liguite qu'on trouve au sud de la baie James, ce qui donne à ces miues d.. lignite une valear incontestable, sans compter les autres nsages auxquels on peut employer ce charbon.

Ces gist:ments de lignite sont presque aussi considérables que ceux de la vallée de la Saskatchewan et du Mackenzie; le charbon qu'ils renferment est d'aussi boune qualité et si les traces d'anthracite qu'ou a trouvées sont l'indication de couches un pen étendues, on peut dire que la région de la taie James est pour le Canada ce que la Peusylvanie est pour les Etats-Unis: un immense bassin de houille. A alle seule, l'exploitation de ces houillières pourrait faire vivre une population considérable et fournir un trafic constant à une ou deux lignes de chemins de fer. Actuellement, lo chemin de fer Intercolonial transporte jusqu'à Québec le charbon bitumineux de la NouvelleEcosse, qui ne vaut guère mieux que le lignite de la baie James, ou plutôt de la rivière Moose; or des mines de la Nouvelle-Ecosse à Québec, il y a près de 700 milles, tandis qu'il n'y en a pas cinq cents de Québec aux houillières de la rivière Moose. C'est done une différence de deux cents milles en faveur de cette derniòre localité, ce qui constitue un avantage étorme pour l'exploitation de ses mines de charbon.

Fer-Certains économistes prétendent que l'Angleterre doit en partie sa prospérité et sa grandeur commerciales aux mines de fer qu'elle possède. Si e'est vrai et si la mème cause peut produire ailleurs les mêmes effets, on pent conclure que la région méridionale de la baie d'Hudson est de toutes les parties du Canada celle qui a le plus brillant arenir. Il y a daus cette région des mines de fer inépuisables et des plus faciles à exploiter. Examinons un peu les localités où se trouvent ces précieux gisements.

(Fer magnétique) "Dans les endroits où la dỷke (sur la rivière Mattagamie, entre le portage de l'Oiseau-Noir et les chutes à la Fourmic) a été récemment enlevée, laissant le mur de roc à nu, celui-ci est doux, plus ou moins perpendiculaire et recouvert d'une couche d'oxyde de de fer juuue. Entre les côtés proprement dits de ces dykes et lo mur de roc, il y a sou vent une donblure ou enveloppe de felsite compact, blanc sale on de couleur fauve et plus ou moins tachetée, ayanr une douce 
fracture conchoidale. Cette enveloppe est évidemment riche en fer, car les surfaces qui se décomposent sont reconrertes d'une croûte pulvérnlente jaune-rougeâtre; soumise à l'action des acides, cette croûte ne fait pas effervescence. A deux places, j'ai remarqué que cette roche contient de petits amas de minerai de fer fortement magnétique." (I)r Bell, Rapport Geologique pour 1875-6, pages 314-5.)

"Le sable de fer' magnétique est accumulé par l'action de l'eau en quantités cousidérables à différents endroits le long de la côte, notamment à la grande et à la petite rivière aux Baleines, aux environs du petit cap Jones et près de la rivière Langlands. " (Dr Bell, Rapport Geologique pour 18-7-8. page 22e.)

"J'ai trouvé des lits de minerai de fer magnétique interstratifiés dans des schistes silicenx, à l'endroit où la rivière à la Truite se jette dans la tête du lac du Genou." (Dr Bell, Rapport Géologique pour 1878-9, page $36 \mathrm{c}$.)

(Hématite rouge ou peroxyde de fer) " La grande proportion de cailloux de quartzite granulaire d'une coulenr très foncée et l'abondance de fragments arrondis d'une hématite silicense, dure, rubannée, contenant en général 50 pour 100 de f'er, qui se trouvent dans les couches diinviennes le long de la rivière Albany, méritent d'être signalées. Ces blocs erratiques viennent probablement d'une grande distance au nord-est, ainsi que cela se voit par leur usure et la direction générale des stries glaciaires. " (Dr Bell, Rapport géologique po:tr 1870-2, page 112.)

(Hémutite brune ou limonite) "Cette localité-1/a Grand-Rapide, sur la rivière Mattagami-est remarquable par la présence d'un grand dépôt de minerai de fer. Ce dépôt se trouve du côté nord-ouest de la rivière, au bas des rapid's. Il forme le pied de la falaise sur une distarce de plus de 300 verges, d'nne manière presque continue, et sa largeur telle qu'exposée, est de vingt à vingt-cinq verges. Les parties les plus hantes s'élèvent à une quinzaine de pieds an-dessus du niveau de la rivière. La surface de ce minerai est tachetée, brune oul jaumo ronjeâtre, et offre à l'wil nne apparence spongieuse on cloisonnée, comme mue grande masse de fer des marais. A la surface, et que'quefois jusqu'à nne profondeur de plusieurs pouces, c'est une hématite brune compacte,souvint renfermée dans des croûtes botyoïdes, arec une stneture à colonnes radiéc, mais plus bas, c'est uu minerai spatique cristallin à grains fins, compact et gris foncé, apparemment pur. L'hématite brune provient évidemmeut de la décom. position du carbonate. L'hématite brune, d'après l'analyse faite par II. Hoftiman, donne 52. 42 pour 100 de fer métallique et le minerai de fer spatique ne renferme que très peu de matiòre insoluble: de fait, il n'y a guère plus, chimiquement parlant, de place pour les impuretés dans ce mincrai de fer spatique, puis qu'il produit me hématite brune si riche..Le sur de calcaire terreux et ennux qui aroisine le minerai est creusé en cavern's rerticales, avec des parties crénelées et arrondies comme les côtés de grandes marmites. Ce mur est quelquefois en partie recouvert par une mince couche de carbonate très ferrugineux. 
Le minerai de fer ne se trouve nulle part en contact arec la roche." (Dr Bell, Rapport Geologique pour 1875-6, page 321.)

(Fer spatique ou fer carbonaté) "Litle Flint, (l'tune des iles du groupe du détroit de Nataskopa) qui a peu d'étendue, est située à trois quarts de mille au sud de l'ile Bélanger. Dans cette lle, le ros plonge S. $80^{\circ} .0$ (mag) < environ $7^{\circ}$, et se compose de quarante pieds de grès gris recouvert par soixante pieds d'argillites et de schistes felsitiques, le tout surmonté par trente pieds de conche de fer spatique manganésifère interstratifié arec uu grès argilleux rerlâtre. Le minerai de fer, qui esi en grande abondance, est répandu en lits minces, rendus noirs par l'action de l'air, et la surface a une curieuse apparence, finement réticulée. Je Dr Harrington a trouré qu'un échantillon de ce minerai ramassé sur l'sle Flint contient $25 \cdot 44$ pour cent de fer métallique et plus de 24 pour cent de carbonate de manganèse. Ces minerais existent en grandes quantités daus tontes les iles du détroit de Nastakopa." (Dr Bell, Rapport Geologique pour 1877-8, page 16u.)

"Les bandes de carbonate de fer spatique formant la roche supérieure (à l'exception du trapp dans les trois lles du nord) do tontes les lles de la chaine du détroit de Nastukopa zonstituent, d'après Harrington, de précienses mines de fer. Ce monsieur a constaté qu'un échantillon, représentant la moyenne de ce minerai, à texture compacte et pris sur l'1le Flint, contient $\mathbf{2 5 . 4 4}$ pour 100 de fer et plus de ringt-quatre pour $r$ ont de carbonate de manganèse. Un échantil. lon crystallisé provenant de l'tle Daviau a donné 27.83 pour 100 de fer métallique. Ces minerais spatiques forment une couche d'une épaisseur royenne d'an moins ringt pieds dans toutes les lles do ce groupe qui, comme je l'ai dit, a une longueur d'environ quatre-vingtdix milles, sans compter les lles situées plus au nord. Cette conche est divisée en lits de quelques ponces d'épaissenr chacun. Lorsque la fracture est fraiche, sa couleur présente les differentes nuances de gris, jaune faure et brun. L'action de l'air sur les parties exposées leur fait prendre la couleur noire et les différentes nuances de brun. Tous les lits ne sont peut-être pas également riches; maics le plus grand nombre de cenx qui se treurent sur le's les que j'ai risitées le sont assez pour constituer un minerai frécieux pour faire du spiegreleisen. Ce qui caractérise ce minerai, c'est son émorme abondance. Formant la conche supérieure sur presque toutes ces grandes 1les, où le plongement est si bas et les strates inférieures ne so voient que dans les falaises du côté de l'est, les conches de fer curbonaté sont répandues sur la plus grande partie de l'etendue de ces fles qui, toutes rémies, forment une aire de plusieurs milliers d'acres carrés. Comme il n'y a pas de bois sur ces lles et comme le roc est beatcoup fratassé par la gelée, etc., le minerai, déjà concassé, pourrait être recueilli en quantités inépuisables. Jes 1 les offrent de hous monillages pour les nurrires et le minerai pourrait être facilcment embarqué en beancoup d'endroits.

"Sur le côté est de l'ile Longue, sur une distance de trois milles à partir de son extrémité sud-ouest, près de la ligne de la marée, an-des- 
sus du grès et du sehiste qui recouvre une roche de trapp compact, il y a des lits de matières très ferrngineuses, dont l'épaisseur varie de dix à quinze piuds. Dans une fle d'environ un mille de longeur et situè à un demi-mille au sud-ouest de l'extrémité sud de l'lle Longue, il y a une bande furrugineuse située dans les mêmes conditions et ure autre plus haut, entre deux épaisses couches de trapp " ( Dr Bell, Rapport Géologique pour $187 i-8$, pages $21 \mathrm{c}$ et 22c.)

M. le professemi Hoffman, qui a fait l'analyse de ce minerai, dit dans son rapport:

"Un échantillou d'une variété compacte venant de l'sle Flint a donné ì l'analyse :

Carbonate ferruginenx.......................... 5270

Carbonate manganésifère........................ 2464

Carbonate de chaux............................. traces

Carbonato de magnésie.......................... 1181

Résiduinsoluble............................ 1094

10009

Fer métallique 2544

" Ce minerai était gris brunâtre et avait une pesanteur spécifique de 3,49. La matière insoluble était blanche et se composait en grande partie de silice. Ce minerai présente min intérêt tout particnlier, à raison de la proportion un peu extraordinaire de manganèse qu'il contient et qui le rend précienx pour la préparation du spiegcleisen. Des minerais semblables sont drpuis longtemps exploités dans plusieurs localités en Europe, mais là les dépôts les plus importants se trouvent dans les terrains deroniens et permiens.

"L'lle Daviau, près de la côte orientale de la baie d'Hudson, est une localité où le Dr. Bell a trouvé du minerai de fer spatique. Un échantillon ramassé par lui, et destructure distinctement crystalline, contient 27,83 pour cent de fer. La proportion du manganèse u'a pas été dléterminéé, mais elle est probablement très forte."

En France, lo traitement de ces minerais de fer spatique, est a peu de chose près le même que celui du fer hydraté; mais presque toujours on y applique la méthode catalane. On les considère commo les plus riches et il n'est pas rare d'en obtenir 0.34 a 0.36 de métal. Lo fer carbonaté, snivant Drapiez, abonde dans certains terrains primitifs; il y forme des couches d'une puissance et d'une étendue considérables.

"Ite minerai du fer spatique ( $\mathrm{FeO}, 0$ ") est anhydre ct contient, lorsqu'il est ptur, 48.275 pour 100 de ter métallique, ordinairement inèlé à du carbonate de protoxide de unanganèse, du carbcuate de maguésie et de la chaux. Il est de couleur jaune ou brun léger. Cassure blanche. Lors: üil est blanchâtre ou de couleur crème, il est à peu près pur, comine à Rosebury, dans le Connecticut. On le regarde alors comme un minerai de première qualité pour la production 
de l'acier En traltant ce minerai arec soin, dans un hant-fourneau à air froid et au moyen d'une manipulation appropriée dans les forges, il donne un fer en barres d'une forc., qui n'est pas surpassée et il se tranoforme en acier avec une facilité extraordinaire...Dans le meilleur fer en barre de Snède, de nểe que dans le fer d'Allemagne provenant du minerai spatique, une quantité de 0.35 de carbone rend ce ser acérain Un minerai de fer manganésifère qui se trouve aux environs de Kuoxville, dans le Tennessee,est extrait de la mine pour être transformé en une très belle fonte blanche miroitante qui, d'apris toutes les apparences, est supérieure pour la fabrication de l'acier à la fonte blanche renfermant du zint, et d'après ce qu'on rapporte, la fonte provenant de ce minerai est pour le moins igale à la fonte blannhe d'Allemange qu'on importe à Troy pour alimenter les uslues où l'on fabrique l'acier Bessmer...La fonte blanche provenant du. minerai de fer spatique est beaucoup em ployée dans ce procédé (la transformation de la fonte en acier). Une des meilleurs sources d'approvissionnement de fonte blanche et grise pour la fabrication de l'acier Bessmer, e'est l'établissement de George Marienhätte, près d'Osuabrük, en Prusse, qui possède des mines de charbon, d'hématite brune et de fer spatique. Le marché sur lequel s'écoulint les produits de ces usines st trouve dans le voisinage. e'est-à-dire daus la Prusse Rhéniane et la Saxe. On emploie principalement cette fonte pour la fabrication de la broche, des tôles de chaudières, de l'acieripuddle et de l'acier Bessmer. A raison de sa grande force, la fonte de inoulage se vend à un prix élevé. L'exportation de cette fonte à Sheffeld, en Angleterre, est considérable et augmente constamment." 1

Le fer carbonaté spatique se trouve en filons dans les terrains primitifs et de transition. Il renferme toujours du carbonate de manganèse ou de magnésie, et fréquemment du carbonate de chaux. $C e$ menerai est mécieu. parce qu'il fournit les fontes blanches lamellaires les plus propres à être transformées directement en acier. C'est à lui que l'Allemagne doit ses célèbres aciers de Styrie et des bords du Rhin, supérieure pour certains usages aux aciers de cémentation. Parmi les variétés de fontes d'affinage, signalons les fontes blanches à grandes lames provenant de minerais carbonates spatiques manganèsifères, monnes a être transformés directement en acier, qui sont produites dans la Styrie, la Carinthie, la Thuringe, la Westphalie, la Savoie et le département de l'Isère." 2

"Ce groupe, ajoute le Dictionnaire de Commerce, c'est-à-dire legroupe sud-est de la France, qui renferme trente-cinq établissements métallnrgiques, se distingue de tous les antres par des procédés métallurgiques spéciaux et par la fabrication presque exclusive des aciers naturels. La fonte est produite dans des hauts foumeanx d'une forme spéciale, par l'emploi exclusif du charbon de bois et de minerais car-

1 Osborn.-Melallurgy of Iron and Steal, pages 47, 61, 115, 57, 895 et 906.

3 Dictionuire de Commerce, all mot "fer" 
honatés spatiques grillés. Cetto fonte, en général blanche et à grandes lames dans sa cassure, est en majeure partie transformée en acier naturel dans des forgus d'affinerie, par une méthode perfectionnée et connue, d'après le nom du principal centre de finbrication, sous le nom de melhode de Rives. Une faible portion de la fonte est transformée en fer malléable par l'affinage comiois. Ce fer est employé à Saint-Etienne pour la fabrication des aciers cémentés.

Les mines des Isles Nastapoka présentent tous ces caractères, et là comme en Suède,en Hongrie et en Espagne, ils forment des couches d'une puissance et d'une étendue considérable. D'aprês les renseignement fournis por le Dr Bell, ces gisements occupent une aire de plusieurs mille acres carrés et les couches qu'ils forment ont vingt pieds d'épaisseur. En supposant une étendue de 5000 acres et un rendement de vingt livres de fer an pied cube de minerai, ce qui est audessous de la quantité ou plutôt de la proportion constatée par M. Hoffman, ces gisements renfermeraient 43,560,000 tonneaux de fer métallique, et si l'on suppose que ce fer vaut sur les lieux mêmes dix piastres le tonneau, ces mines représentent uue valeur de $\$ 435,600.000$.

Nous attirons tout spécialemeut l'attention sur ces chiffres, qui patr. ont extrnordinaires à cenx qui ne se donneront pas la peine de se resdre compte des faits.Depuis quelques années,on parle tant de mines et les lanreurs de spéculations sur les mines ont fait tant dupes daus ce pays, qu'on a fini par ne pas croire à l'existence de nos richesses minieres. Dans ce cas-ci, il est facile de se former une opinion exacte. Les couches sout à peu près uniformes en richesse et en épaisseur et eiles reconvrent un groupe d'lles roisines du détroit de Nistapoka et s'étendant sur une distance de quatre-ringt-dix milles. L'épaisseur de ces conches est d'une vingtaine de pieds et elle est d'autant plus facile à constater que les lits ferrugineux sont à la surface du sol.

Cette circoustance donne une raleur particulière aus mines de Nastakopa. Dans la plupart des mines, nous pourrions mesme dire dans toutes les mines de fer, à l'exception des gisements d'hématite, il faut crenser, ourrir le roc et fitire des travanx considérables pour atteindre le minerai. Ici, il n'est pas nécessaire de faire ces travaux dispendieux; Ie minerai se trouve à la surface du sol et la geléo a tellement désagrégé la roche qu'elle est fricassée, comme dit le Dr Bell, en sorte qu'il n'y a qu'à la ramasser pour la transporter où l'on reut. Puis il y a partout de bons monillages pour les narires, $c 3$ qui complète les facilités d'exploitation de ces mines inépuisahles. Si on trouvait plus avantagenx de ne pas fondre le mineari sur les lieux mêmes. on pourrait le transporter en Augleterre, ou dans la province de Québec. Du détroit de Nastakopa a Liverpool, la distance ést de moins de 2,700 milles. Dn mêmu détroit au fort Moose, il n'y a qu'environ 600 milles, et du fort Moose à Québec, à peu près la mểme distance. On parle depuis longtemps de continuer le chemin de fer de Quebec et du lac Saint-Jean jusqu'à la baie James, et la construction de ce chemin, qui pourrait se faire dans les conditions les 
plus faciles, déterminerait probablement l'exploitation des mines da Nastakopa, qui pouraient ì elles seules fournir un immense trafic à ce chemin de fer. D'ailleurs, le bois et lo liguite qui abondent dans lia région de la rivière Moose permettriient de fondrenvantagens'ment le minerai sur les bords de la baie James et de ne trausporter en chemin do fer que le métal prẻt à employer dans l'industrie métall urgique.

Ce qui précède montre clairement que la région de la baie d'Hudson est tres riche en minéraux susceptibles d'ètre explontés, notammant le cnivre, sur les bords du lac Abatagomaw; le lignite,dans la vallée de la Mattagamie; le fer spatique, sur les bords de la mẻme rivière; le fer magnétique sur les bords du lac du Genou, qui n'est qu'une expansion du cours de la rivière Hill; le fer spatique, dans les lles dn détroit de Nastakopa, et le mauganèse, qui fait partie de ce minerai; la galène sur les bords de la petite rivière avix Baleines, et de la mer, jusqu'à l'entrée du golfé Richniond, un peu au sud des Iles où le minerai de fer abonde. Cette deruière région n tout ce qu'il faut pour devenir le plus grand centre de l'industrie minière au Canada: il ne lui manque qre les grandes roies de communication par terre, et ces grandes voies de communication ne manquerout pas de se coustruire quand on seru bien convaincu de l'importance et de la richesse àu pays qu'elles sont destinées à ouvrir à la civilisation.

\section{OHAPITRE VII}

LES REGIONS COLONISABLES-LEUR ÉTENDUE-CE QU'ELIES SONTRÉCOL'TES, SOL, BOIS, MINES, CLIMAT-LEUR AVENiR.

Pour compléter ces notions sur la grande contrée que uous voulons faire connastre, nous croyons à propos de citer les différentes opinions do cenx qui l'ont risitée on exploitée, en domnant d'abord tos appréciations qui s'appliquent is tout le' territoire,puis cellons qui : 'ous? trait qu'd chacune des trois régrions qui, au point de vue du clims dn sol et de ses produits, forment des parties distinctes et bien caractérisées.

Territoire de la baie d'IIudson.- " Relativement anx prodnits régétaux, je pense que tous ceux qui croissent en Russie pourruient être enltivés avec profit dans le territoire da la baie d'Hudson. Tous les produits naturels que nous importons de Russie, tels que le lin, le chanvre et les céréales, pourraient étre cultivés d'une manière profitable et avantageuse dans sertaines parties $d u$ territoire qui environne la baie d'iIndson. La salsepareille pousse comme plante indigène et de qualité supérieure dans ce pays. Nous en importons chaque année 180,000 livres do Russie, de Honduras et d'autres eudroits. Chaque aunév nous importons de Russic 40,000 galons de canneberges qui abondent tout autour de la baie d'Hudson. Je ne doute guère qu'un graud nornbre des régious accessibles abondent en riehesses métalliques d'une infiuiment plus grande valeur que tous les reveuus que le commerce des pelleteries est susceptible de produire." 
Cet extrait est tiré du témoignage donné devant un comité de la Chanbre des Communes en Angleterre par M. Isbister, qui a dmeuré dans le pays dont il parle, l'a parcouru en tous sens et étudié arec soin.

" La première région et la plus étendue est celle qui s'étend depuis la côte de Labrador, en faisant le tour de la baie d'Hudson, jusqu'aux terres arctiques, vers le nord. Cette région est entiêrement une région forestière, capable de fournir en immense quautité le bois, qui forme une partie si importante du commerce d'exportation du Canada. Ces interminables forêts se composent principalement d'épinettẹs de fortes dimensions. On peut se former une bonne idée de la salubrité du climat par le grand âge auquel parviennent beaucoup des employés de la compagnie, qui passent leur vie dans ce pays. Je n'ai entendu parler d'aucune maladie à laquelle les blancs sont exposés, sauf la gottre " (McLean's Hudson Bay)

Il serait inutile de multiplier ces citations, qui ne fout que confirmer d'une manière générale ce que nous avons exposé d'une manière plus détaillée dans tout ce qui précède. Examinons plutôt ce qui a été écrit respectivement sur chacune des trois grandes régions dont nous avons indiqué les limites à grands traits, en décrirant les pricipales rivières : la région de l'East Main, ou de l'est; la région de la rivière Moose, ou du sud, la région des rivières Nelson et Churchill, ou de l'ouest.

Région de l'est - Elle comprend tout le plateau égoutté par les rivières qui se jettent dans la baie James et la baie d'Hudson, à l'est, entre le 60 e digré de latitude au nord et la rivière Nottaway au sud, ce qui renferme la contrée du lac Mistassini. C'est par excellence la région des mines; c'ust là que se trouvent ces gisements de fer et de plomb, dans les environs du golfe Riuhmond, et de cuivre, dans le roisinage du lac Abattagomaw, près du lac Mistassini, qui offrent à l'industrie minière un si vaste champ d'exploitation. Les forêts d'épinettes blanches renferment aussi d'imenses quantités de bois suscep. tibles d'être exportés.

La plus grande partie des planches d'épinette blanche qui sont exportées de Norvège en Angleterre ne mesurent que cinq pouces de largeur, d'après le témoignago donné par M. Stuart Thaine devant le comité de la colonisation et de l'immigration en 1878. •

Ce monsieur dit aussi que c'est dans les forêts de la Norrìge qu'on prend la plus grande partie des bois d'épinette qui s'emploieut en Angleterre. Cir les forêts de l'East-Main peuvent fournir des bois bien supérieurs à ceux de la Norvège,puisque mểme au fort George, situé à quatre cent cinquante milles an nord du fort Moose ou de l'extrémité sud de la baie James et jusque sur les confins de la limite septentrionaie des forêts, on fait en grandes quantités des billots d'épinette qui mesurent vingt-quatre ponces de diamètre, ainsi que cela

\footnotetext{
- Rapport du comité permaneut de la colonisation et de l'immigration, 1878 page 122.
} 
est constaté par M. le Dr Bell. S'il y a sur les bords de la GrandeRiviêre des forêts où l'épinette peut clonner d'aussi beaux billoto, il est évident que ces forêts se continuent au sud jusqu'a la riviêre Notaway, puisqu'en allant dans cette direction le sol et le climat s'améliorent et sont hien plus favorables à la croissance des bois. La distance entre ces deux rivières est d'environ trois eents milles; si la lisière occupée par ces forêts d'épinette a seulement une largeur de trente milles - c'est la largeur des bonnes terres, d'après ce qu'on a rapporté an Dr Bell-ces forêts d'épinettes de l'East-Main occupent une aire de 9000 milles on 5760,000 acres en superficie. C'est-di-dire qu'il y a là une rógion forestièrt presqu'aussi considérable que celle du Saint-Maurice.

Les terreins agricoles sont moins étendus. Lo sol ef assez bou dans presque toute la région des forêts; mais on prétend qu'au délà de la rivière Fast-Main, le clinat constitue une obstacle sérieux à l'exploitation agricole, qui se trouveriit ainsi circonscrite ì un territoire fort restreint dans la vallée des rivières Rupert et Natoway. On peut estimer sans crainte à 1000 milles carrés, ou 640,000 acres, l'étendue de ces terrains cultivables. Le climat n'est pas assez chaud pour faire mûrir le blé ; mais l'orge, l'avoine, les pommes de terre et toutes les légumes viennent à perfection. La compagnie de la baie d'Hudson ezploite depuis bien longtemps une ferme où ellélèvedes bêtes à cornes, des moutons et des cochons, cequi prouve qu'on récolte sur cette ferme les grains néees saires à l'alimeutation de ces animaux. Les ter. rains bas qui avoisinent le rivage de la mer produisent en aboudance l'herbe et le foin saurage dont on nourrit le bétail en hiver. L'établisement de la compignie de la baie d'Hudson à l'East-Main, dit le Dr Bell, est maiurenu pour faire l'élérage du bétail. Les bêtes à cornes et les montons que nous avous vus là étaient eu excellente condition. Ailleurs, il dit que cette région furait un bon pays à pâturages. Quant à la température du cette région, en printemps et eu autorane, on peut s'en former une idée par ce qu'en dit Dobbs à la page 12 da son livre sur la baie d'Hudson :- "Le capitaine Gillam entra dans rivière liupert lu 29 septembre .. Le 9 décembre, ils furent pris par les glaces dans la rivière... En avril, 1769, lo froid était déjà fini... En 1770, la glace parut sur la rivière Rupert le 10 octobre, mais il y ent du temps chand après cette date... La riviêre prit le 6 novembre. Vers le 20 mars, le dégel commença... Sur la rivière, la glace était fondue le 20 avril. "-La date de ces phénomines est à peu prìs celle des mêmes phénomènes dans la province do Qnébec, et s'il faut juger par là de la température clurant le reste de l'aunée, on est bien forcé d'admettre que cette région est tout à fait susceptible de culture.

Si l'agriculture offre certains désavantages daus la région de l'EastMain, elle présente aussi de's nrantages incontestables. Si les miues, les forêts et les pecheries, qui renferment tant de sources de richesses à exploiter dans cette coutrée, finissent parattirer uno certaine population ouvrière, il estérident que les produits de la ferme auront une graude valeur à raison de la proximité des endroits où ils seront requis pour la 
consommation. Alors ou verra sans doute de bravas pionniers da la colonisation s'empresser de livrer a la enlture les millions d'acres de bon sol arable qui se trouvent sur le bords de la meret que l'ignorauce a fnit classer jusqu'moujourd'ui daus la catégorio des terains incultivables et in habitables. Il arrivera pour le territoire de l'East-Main ce qui est arrivé dans notre proviuce pour celui du Sagnenay : pendant longtemps, jusqu'ti 1850, grâce aux rapports inexacts des traiteurs, on a regurdé toute la vallée du lac Saint-Jean comme un pays inculte on inhabitable et un deces traitenrs alla même jusqn'à dire à un employé du département des Terres de la Couronue que ce pays avait si pen de valeur, qu'il serait inutile d'y transporter une menle ou nne scie de monlin. Des explorations faites avec soin ont mis à néant tousces rapports mensongers on exagérés et aujourd'hni cette prétend ne région stérile est regardée à juste titre comne nue des plus belles parties agricoles de la province; clle est habitóe par une population de près de trente mille annes, sans compter que nos jetnes cultirateurs n'attendent que l'ouverture du chemin de fer jusqu'aux bords du lac Saint-Jean pour aller s'y établir en masse.

Région de i'ouest.-Elle renferme la vallée, ou plutôt le plateau qu'égouttent les rivièresChurchill et Nelson. Ce platean a une étendue d'environ 242,000 milles, on 188,800,000 aeres en superficie. De cette étendue, environ 73,600 milles ou $47,104,000$ acres sont cultivables et habitables, sous le double rapport dn sol et du climat. Ceci exclut les deux tiers an moins de la région traversée par la partie inférieure de la rivière Churchill et celle qui avoisine la rivière Nelson en approchant de la mer. L'aire cultivable est $r$ riséo en deux parties distinctes: celle de la rivière aux Castors en celle de la région des lacs, située au nord et à l'est du lac Winnipeg.

Les ferrains cultivables que nous comprenons cians la région de la rivière aux Castors s'étendent jusqua anx environs du lac de l'sle à la Crosse, et forment me aire de 12,000 milles ou $7,680,000$ acres carrés, environ la moitié de tonte la superficie du Noureau-Brunswick, qui est de 27,700 milles carrés. Daus cette contrée de la rivière aux Castors, les céréales ordinuires : l'orge, l'aroine, lo seigle, les pluntes racineuses, les légumes et lous les régétanx vieument à perfection; l'on récolte nême le blé suns difficulté daus benucoup de localités. Á. l'ile à la Crosse, à Athabaska même, écrit Mgrr Taché, en défrichant les bords des lacs, on est certain do la récolte du froment et des légumes. Le sol de la contrée de la rivière anx Castors, dit Macdonald, est bon et fort, bieu boisé, fortement boisé de rringnifiques épinettes et d'autres bois précieux.... Le fort (de l'sle à la (rosse) est propre et compacte, le terrain enviromant est bas ct marécageux. La pèche dans le lac est facile et donne un approvisionnement constant de nourriture saine et fraiche, l'été comme l'hiver. La petite fermo est productive et les quolques bestianx qu'on y gardait étuient en excellente condition. (*)

(") Peace River-A canoe royage from Hudson's Bay to Pacific, p.o.ges 53 et 57. 
Sir Johu Richardson dit que !a rivière aux Castors égoutte une petito étendne de prairie et d'après la deseription qu'en donue le capitnine Paliser, cette région est bien hoisée et aussi fertile que la vallee du bras nord de la Suckntihewan, qui constitue la partie la plus riche des beatux terrains désicnnés sous lo nom de Zone ferlale.

Le professenr Maconn, de la cornmission géologique, a parcouru en 1875 toute la région comprise entre le portago Méthy et le fort Carlton, en passant par le lac: Vert. Dans son rapport, on trouve les notes suirantes :

"Je remarquai que les pommes de terre (qu'il asheta d'un vienx métis) poussaionnt dins un terrain qui n'avait étó dépouillé que le printemps de la forêt yui le reconv rait... On avait récolté do l'orge l'année précédente, d'où il faut concelure que l'orge et les pommes de terre murissent anx environs dn lac Móthy. La gelée avait attaqué les pommes de terre lo 9 (Ae septembre); mais j'ai appris ensuite qu'olle les arait pareillement attaquéus dans la province de Manitoba le 21 noût, ou dix-neuf jours plus tôt qu'an Portage-la-Loche. (1)

"La rivière Crense et le lac do l'sle à la Crosse sont tons deux environnés par des forêts de trembles, ce qui dans le nord indique toujours nu bon sol. Le fort est agréablement situé sur un bras du lac.. Du cóté droit, ell face du fort, se trouve la nnaison du missionnaire eatholique...J'ai fait un examèn soigné des environs et j'ai été extrèmement surpris de voir que les pommes de terre étajent encore verdoyantes et même que les fèves ramenses n'avaient pas été attaquées par la gelée (le 22 septrubre). Les végétaux de tontés sortes viennent bien; l's navets, lis pommes de terre, les carottes et les chonx étaient de fortì grossenr. Le blé, l'orge et l'avoine rénssissent bien : ruais le llé n'est pas regardé comme une récolte certaine, quoiqu'il semble que la gelée ne fair jamais dommage à rien. Aucuus des végétanx cultivés ne proviennent de semences hâtives, et si on employait des sémis d'espèces hatives, la production des végétaux, dans tons les endroits de cette région du nord, serait toujours une affaire certaine. A la nission, on cultive le blé et les autres céréales et l'on a bâti un moulin à farine de quatre chevanx vapeur pour moudre le blé récolté dans le voisinage. Lee blé d'automne devrait réussir ici, vu que la neige reconvre le sol tant qu'elle n'est pas fondue par les fortes chaleurs du mois d'arril... Apparemment, il tombe ici beancoup plus de plnie que dans la région de la vivière à la Paix, et il y a peut-être moins de chaleurs, ce qui fait que les récoltes arrivent probablement pius tard is maturité. 2

"Dans le voisinage du las,.Vert, le sol est d'excellente qualité...A u fort, la ré:olte do pommes de terre a uxcédé 500 minots cette année. L'orge vient bien, mais le blé est une récolte douteuse. La gélée du 8 septembre a été très forte ici et elle a endonmagé les pommes 
de terre, ce qui montre quo le climat ost plus froid que plus au nord. Jusqu'à cette date (la fin de septembre) la tempórature a été superbe et tout à fait chaude; il y a en la nuit quelques gelées légères, mais pas assez fortes pour détruire les flenrs ou arrẹter la végétation. Cetto région est partont propre ì fuiro des étublissements; lo sol est de premiere qualité et tout à fait see. Il y a des myriades de poissons-poissons blanes-dans lo lac de la rivière..."

Il fandrait ôtre bien exigent pour s'obstiner à ne pas classer cette régrion de la rivière aux Castors, du lae de l'yle à la Crosse et du lae Vert parmi les bonnes régions colonisables du Nord-Ouest. La températue d'été est belle et réellement ehande, le sol est presque partout d'excellente qualité, les forêts renferment en abondance des trembles et des épinettes blanches de la plus bello venue, d'assez grandes dimensions pour faire de bons bois de construction, les lacs et lès rivières fourmillent de bons poissons, les córéales, même le blé, ainsi que les légumes et les régétaux de toutes sortes, viennent à perfection, tel que l'atteste lo témoignuge do tons ceux qui ont visité et qui comnaissent ce pays : que peut.on exiger davantage pour faire des établissements prospères?

Eh bien, il y a dans cette région, $7.680,000$ acres de ce beau terrain agricole. C'est assez, mème en faisant largement la part do tous les incour énients, de tous les décomptes possibles, pour établir une po. pulation agricole de 300,000 âmes. Narons-nous pas raison de classer cette contrée au nombre des régions qui méritent d'attirer l'attention?

L'espace compris entre cette rérion ot la rivière Nelson renfermo en jlusieurs endroits de belles forêts d'épinettes et do bons terraius; mais comme on n'a pas de renseignements complets et détaillés sur la nature du climat, du bois et du sol, nous rangeons pour le moment cet espace dans la catégoric bien trop exagérée des régions inhabitables. D'ailleurs, il y a suffisamment de belles terres colonisables immédiatemerit an sud, sur les bords de la Saskatchewan, pour continuer la suite des établissement jusqu'à la partio nord du lac Winnepeg.

La contrée que nons appellerons la région de la rivière Nelson, est entourée par une ligne partant de la Pointe-aux.Monsses, contomruant le lac de l'Ile et la rallée de la rivière Shammattawa. jusqu'à une centaine de milles du rivage de la baie d'Hudson, puis reunontant au nord de lin riviere Nelson jusqu'à son point de depart. L'aire comprise dans ces limites forme une éteudne d'à pen près 61,600 milles ou 39.424,000 acres carrés, à peu près le tiers de la superficio totale de lis province d'Ontario et plus du quart de celle de la province de Québec. Les terrains siluriens et les formations laurentiemnes et huroniennes se partagent ce territoire à peu près par égales moitiés; mais même dans ces dernières formations, il y a d'immenses étend ues de bon terrain, surtout de terre glaise. La température de la saison agricole n'est guère plus basse que celle de la. Rivière-Ronge et partont les

- Idem, pago 181 
céréales-même le blé en certains endroits, et les végétaux de toutes sortes riennent ì perfection. Les forêts renferment d'immenses quantités de bons bois de construetion, surtont l'épinette blunche, le pin de Bank et l'épinette rongo, sans compter le tremble, lo peuplier et lo bouleau, qui peurent fournir le combustibie el les bois pour les usages domestiqnes. notamment pour cloturer les terrains.

Lo fort Norway est le point le plus marquant de toute cette con. trée et il est pour ainsi dire habité par une population agricole. Le pays qui environne ce fort est plutôt ondoyant que montueux et le sol est bon en plusieurs endroits. La pêche et la chasses sont abondantes. Jiéturgeon, pesant jusqu'r cent livres, le brochet, et surtout le poisson blanc, fourmillent dans le lac et dans les rivières. Sur les ilets rocheux du bean lac Playgreen, le gibier est si abondant qu'on ranasse les cuffs à pleins canots pour les transporter an fort Norway.

Mais ees ressourees, si précienses qu'elles soient comme aecessoires, ne suffirient pas à rendre le pays habitable, si le sol et le climat n'étaient pas bien meill'urs qu'on ne le suppose genéralement. Sur ce poin, il est facile de citer des témoignages qui ne laissent aucune place aux doutes ni anx préjugés. Le facteur en chof M. Macdonald ét G. Gladman, qui ont habité lo pays, le Dr Bell, qu l'a explorí et étudiés arec cet esprit d'observation et cette justesse d'appréciation qui le distinguent, s'accordent tous pour représenter cette région comme un pays agricole d'une valeur incontestable.

"On récolte, dit Gladman, de bonnes pommes de terre au fort Norway et au village des Sanvages, qui se : ronve dans les environs. Les Sanvages ont bâti de bonnes maisons do bois équarri, et eultivé des morceanx de terre. Ils sont industrieux et deviennent gruduellement indépendants de la chusse aux pelleteries. Le sol, an village, est asse\% bon. Jo ne sache pas qu'on ait cultivé le blé; mais comme cette localité se trouve sur le mème parallèle que le fort Cumberland (où la culture du blé réussit bien) on pourra cultiver ce grain quand il aura été fait des défrichements plus cousidérables.

"De 142 à 1845, je fus stationné an fort Oxford. Je n'éprourai pas ln moindre difficulté à récolter des légumes et des pomines de terre en assez grande quantité pour les besoins des gens de la compagnie de la baie d'Fundson, et il en restait encore pour le fort York et pour les Saurages" •

Nons arons cité à la page 22 l'appréciation générale que le Dr Bell donue da sol qui convre tonte la région de's rivières Hayes et Nelson. Dans un rapport spécial adressé an ministre de l'intérieur, il s'exprime d'une manière aussi farorable. "J'ai déjà mentionné, dit-il, le beau sol de glaise qui couvre la noitié eupérieure de la vallée de la rivière Nelson. Tons les rapports s'aecordent à dire qu'un sol de glaise également bon, avec de rares turgeseences rocheuses,s'étend vers le nord-onest dans la région égouttée par la rivière au Bois Brulé et

(`) Rapport du comité chargé de s'enquérir des droits de la compaguie de la baie d'Hudeun, page 5. 
sur toute la distance jusqu'à la rivière Churchill... Ondit que la bonne terro forme une aire considérable an sud de la route des bâteanx (les rivieres Franklin, Hill, d'Acier et Hayes), y compris la région enviromnant le lac God et le sonrs supérieur de la rivière Serern. Que la sol est fertile, c'est ua fait qui est pronvé par les jardıns dụ fort Norway, du lac Travers et du fort Oxford, autant d'endroits où l'on fait $\mathrm{d}$ f tres-belles récoltes de plantes-racines et de légumes. L'orge mû 'it wien vers le nord-est, an moins jusqu'an fort Oxford. Jes ecpèces de bcis les plus ntiles dans cette région sont l'épinette blanche et l'épinette rouge, que l'on woure jusqu'an delà de la vivière Churchill, ainsi que le cyprès, qui. se rencontre presque jusqu'aux environs du fort York. Ces especes seraient toutes bonnes pour faire des traverses de chemins de fẹ et des poteaux de télégraphe ainsi que da bois de chautrage."

Dans son rapnort à la commission géologique, le Dr Bell entre plus dans les détails et domn, assez de renseignements pour mettre le lecteur en position de se former nue juste idée de tontes ces régions. "En général, lisons-nons dans cet excellent rapport, les forèts et la flore de la rógion de la rivièro Nelson iadiquent nu elimat flus donx que celui du la régrion correspondinte de l'autre côté de la baie d'Fudson. Ceci parait résulter, an partie du rooins, des rents de sud qui prévalent en été et apportent l'air ehand. probublemont de la rallée du Mississipi, jusque lans cello de in rivière Ronge et ai-dessus du lac Winnipeg, dont la cempérature est élevée et uniforme durant less mois d'été. Cela provient aussi de l'absence des gélées d'été dans la région du fort Norway, qui paraft jonir d'un climal pour le moins aussi bon que celui de la prorince de Manitoba. Las petits frnits, les concombres, les meions mus qués at les légumes de tautes sortes parviennent à maturité au fert Norway. L'orge est une récoite sure. Jusqu'au jourd'hui, comme il n'y arait pas raison d'essayer la culture du blé, l'éxpérimentation de cette culture ne parait pas avoir étó faite dans cette région : mais il est très probable que sette culture réussirait, va qu'il est de fait que le blé mûrit parfiatement dans la région de l'A thabaka et de la riviène à la Paix, ras s des localités situées à plus de mille milles plus au nord-ouest.

"La rivière Nelson porte pers la mer la haute tempéature que les enux du lac Winniperg reçoivent on partie de celles des rivières venant. du sud et de l'ouest, ee qui a pour elfét de faire pousser dans la vase glaisense de ses berges un forte rúgétation do rospaux, de joncs ei une grande variété de plantes aquatiqnas. Le climat de cot te région est agréable en été, sans excis do pluie, et la tempèrature d'hiver, ynoique t' oirle, est représentée coinine étant pure, uni orme, avec genlenent uno quantité modérée de neige. La terro serait facile à défricher, et qand on ensidere l'anprovision nement illimité de bois pour la construction, le chanflige, et la bonne ecu qui se trovive partont,

(*) Zapport du ministre de l'Intrieur jour 1858, pages 9 ol 10. 
dans laquelle on trouve cul abondences une variété d'excellent poisson, ainsi que la plus grande proxinnité a laquelle cette région se trouve de l'Europe, on arrive à la conclusion que ce pays offre anx -immigrés quelques avantages qui ue se rencontreul pas dans la région des prairies située plus à l'ouest.

"A u fort Oxford, l'orge, les pois, les fères, les plantes-racines, les légumes et le foin réussissent bien et la région environnante p arraì faire un beau pays de nétaieriè et d'élévage. Mème au fort $i \mathrm{crk}$, la pomme de terie et plusieurs espèces de légurnes peuvent itre cultivées avec succès."

De tont ce rui précède, il faut bien conclure que la répjion qui nous occupe constiune indubitablemeut un bon pays agricole. Le sol est excellent partont. Il se compose de graise et de débris alluviens qui forment là comme partout ailleurs une excellente terre à grains et à paturages. Les terrains glaisenx font croftre l'herbe, le trêfle et le mil en abondance. C'est sux ces terrains que daus la province de (Nuébec, dalls la vallée du Saint-Laurent coinme dans celle du Richelier, on récolte ces immences quantités de foin qui fournissent à la consom. mation localo et alimentent en sus un énorme commerce d'exportation aux litats. Unis. L'orge, l'aroine et les pois vienment en abondances dans ces terrains glaiseux. Pour ce qui concerne les paturages, la région de la rivière Nelson a sur les prairies des rivières Rouge et de la Suskatchewan l'immense arantage d'être bien fournie de bonne eau. Ontre le fort contingent des lacs ot des rivières, l'eau se trouve partout, dans la contrée sitnée an nord-est du lac Wiunepeg, à une profonder d'une quinzaiue do pieds sons la surface du sol; cette ean est pure et d'excellente qualité, ce qui n'est pas toujouri le cas à l'ouest de ce lac où l'on a quelquefois beancoup de difficuté à trouver un endroit propre à creuser un puits.

T.es forêts de la régrion située à l'est du lac Winnipeg sont un autre avantage que cette région possède sur celles de's prairies. Duns la proviuce de Quebec, le défrichement contie enviros dix piastres i'acre. et il ne derrait pas coûter plus daus la contrée traversée par les rivières Hayes et Nelson. Lih bien, an prix que vaut le bois dans cette contrés vousine des grandes prairies, où il n'y en a pas, la forêt qui re. convre le sol vaut bien plus que le cont du défrichement. Puis, tandisqu'en hiver le colo" de's prairies u'a rien à faire, ne peut rien trouver à fuire qui soit pruit.ible, celui des régions boisées a ses foréts à exploiter et troure la line sonrce de richesse pour le moins érüles à celles que donue l'agroiculture. Le fort York est moins éloigné de Liverpool que Qnébec et la descente des bois par les rivières Hayes et Nelson n'est certuinement pas plus difficile sur ces rivières qu'elie l'est sur l'Ontaouais et le Saint-Manrice, par ou passent presque tous les bois qui sont deseendus à québec potr être mis a bord res navires quiles tramsporteut en Ingleterre. L'exploitation des forêts de

- Dr Bell.-Rapport GCologigue pour 1877-i8, pagea 29cc et 30cc. 
la rivière Nelson, qui renferment en immense qưantité de l'épinette blanche propre à faire du bien plus beau bois que celui qu'on importe de Norvìge en Angleterre, est done aussi fucile et éridemment moins dispendiense que celle des forêts de lit province de Québec. Arec cette perspentive en rue, le colon pourrait-il raisonnablement manifester çuelquo rópugnance pour ces terrains, quand il est hors de donte yu'ils forment un sol d'une richesse incontestable!

La températura ne permet pas de cultiver le blé. C'est un inconvónient, assurément, mais un inconvénient bien moius considérablo quon ne le suppose généralement. Après tout, le blé ne constitue qu'une partie comparativement médiocre des produits agricoles. 1)'après le recensement de 1871, dans la province de Québec la récolte totale des céréales, blé, orge, avoine, seigle ct pois, est de $21,507,100$ minots, dont 2,058.076 minots de blé, ou 9.52 pour 100 de la récolte totale. Comme on le voit, la culture du blé ne constitue pas un dixiène de la culture des céréales et pas un vingtième de toutes les opérations agricoles. L'tbsence on l'impraticabilité de la culture du froment ne rend donc pas une région impropre à la culture, et mème arec ce désavintagé, la cuntrée que traversent le cours supérienr. des rivières Hayes et Neloum n'en est pas moins m beau pays agricole, offrant des arantages incontestables à la colonisation.

Mais, dira-t-on, dans la région de la rivière Nelson, la surface du sol ne dégèle en été qu'a deux on trois pieds de profondeur et plus bas in gelée reste en permanence dans la terre.

Ii est rrai que dans les enrirons du fort York, on troure la gelée à trois ou quatre pieds sous terre en été. Mais cela ne se roit pas à tune centaine de milles en allant dans l'intéricur des terres. A cette distance de la mer, on tronve aussi de la gulée dans la terre, même durant l'été, dans les inurécag'es on sur les bozds des rivières, dans les endroits où les rayous du soleil ne peuvent pas pénétrer; mais cela se voit pareillement dans d'autres parties du Canada où lo climat n'offre aucun obstacle â l'agrienlture. L'été dernier, dans le commencement du moib da juilllet, ì noins do denz milles de Québsc, il y avait sur les bords de la rivière Siaint-Charles un banc de neige cui n'était pas uncore fondu. Inntile do dire que lo sol était cornplistement goló sons ce bane de neige, te qui n'empèche pas qu'ì cent pieds plus loin, dans un terrain exposé aux ruyons du soleil, il y arait 1 u clianp de grain qui avait la plus belie apparence. Celui qui aurait conclu de l'existence de ce bane de neige que les bords de la rivère Saint-Charles no soni pas cultivables, parce que la gélée reste dans' ol en certains endroits anrait done commis me erreur grare. C h th est de même de la glace que l'on rencontre occasionnellement sur les bords de la riviòro Nelsou: il pent se faide que le sol reste gulés en été dans les endroits où il est inaccessible anx rayons solaires, mats partont ois il est exposé an soleil, il dégèlo complète. ment dans la partio cultivable de cette région.

Enfin, qu'on l'examine à u'importe quel point de vae, cette région est parfaitement habitable et cultivable, et même eu retranchun les 
deux tiers de son étendue pour faire largement la part de l'imprévu, il reste encore 13,141,333 acres à livrer à la colonisation. C'est à peu près l'étendue de tous les terrains occupés de la province de Québec, qui est habitée par une population excédant treize cent mille âmes Ou pourrait donc former une bello province dans cette région, dont les rivières forment en plusieurs endroits de bonnes lignes de navigation. En supposant que les $13,141,393$ acres de terrains cultivables soient occupés par une population la moitié noins dense que celle de la province de Q r. . wec, ì y a là suffisamment d'espace pour établir ou plutôt faire vivre près de 700,000 personnes. C'est autant que la population collective de la Nonvelfe-Ecosse et du Nouveau-Brunswick, qui n'étaient habités en 1871 que par 573,344 âmes.

Région du sud-Ill serait pent-être plus exact de l'uppeler " région de la baie Janes," ear nous comprenons dans ses limites toute l'immense plaine dont les eaux s'écoulent dar" cette baie, par la vallée de la rivière Severn qui a sou embonchure dans la partie sud de la baie d'Hu.'son. De l'est à l'ouest, e'est-à-dire des sources de la rivière Hannah à celle de la rivière Severn le territoire a huit cent milles de longueur, et sa plus grande largeur, cntre le cap Henriette-Marie et la tète du lac Long, excède quatre cent inilles. Sa superficie totalo t. d'environ 245,0,0 milles, ou 148,800,000 acres carrés. Cette étendue excède de 51,645 milles carrés la superficie de la province de Québec, qui n'est que de 193,355 milles, ou $123.7+7,140$ acres carrés.

Cornme de raison, il faut défalquer les parties que la climat ou la mauvaise qualité du sol rendent impropres à la culture. Au point de rue du climat, il faut retrancher tout l'espace situé an nord de la latitude du fort Albany, bâti à l'erubonchure de la rivière du même nom. Cet espace occupe une aire d'environ 45,000 milles carrés. Si on y ajoute une douzaine de milliers de milles par la partie extrême de l'est, on forme un total de $\$ 0.000$ milles. Quant à ce gui regarde le sol, il faut défalquer, en certains endroits, la lisiere rochense on peu fertile qu'avoisine la hauteur des terres ou la iigne de paitage des caux entre le bassin du Saint-Laurvnt et celui de la baie d'Hudson. Ces terres de peu de valeur, comme sol agricole, ont uue étendue d'à peu près 15,000 milles carrés, en mettant le plus haut. ehiffre. Ces défalcations faites, 11 reste done pour la partie cultivable, sous le double rapport du sol et du clinat, une étendue de 170,000 milles, on 108,800,000 acres en superficie.

Dans tout cet espace, le niveau du sol est uniforrae, à peine accidenté en do très rares cudroits par des collines le peu d'étendue et dont la harteur n'excède nulle part cinq cents pieds. Près de la hauteur des terrus, qui forme la limito sud de ce territoire, l'élévation du sol an-dessus du niveau de la mer varie entre neaf ot douze cents pieds. Oetto élévation diminue graduellement en allant rers la baie d'Hudson, et à une centaine de milles de la hauteur des terres, elle u'atteint pas quatre cents. Dans le sens longitudinal, le niveau de cette grande plaine ne forme qu'une pente irrégulière et uniforme d'une centaine de pieds, en allant vers l'ouest, sur une dis. 
tance de cinq à six cents milles. Entro l'extrémité nord du lac Winnipeg' et le lac Abittibi, ésoignés d'environ huit cent milles l'un de l'antre, il n'y a qu'une différence de nivean de 147 pieds: le lac Abittibi est éleré de 857 pieds an-dessus du nireau de la mer, et le lac Winnipeg de 710 pieds. Il serait difficile de trouver un pays plus plan et moirss accidenté.

Le platean égoutté par les diflérentes riviéres qui forment le réseau hydrographique de la rivière Moose - les rivicir... Abittibi, Mattagami ot Missinaibi-forme une étendue d'ì peu, $38,400,000$ acres, ce qui excède un pen la zurface de la vallée de l'U an auais. L'aire égouttée par la ririêre Albany est un peu moins grande et ne couvre quo 23, 000,000 d'acres. Le cours ce cette rivière, en suivant sa branche sudest, a plus de 500 milles de longueur et elle est navigable jusqu'à Martin's Falls, à 250 milles de la mer.

Maintenant, cette immense région est-elle cultirable, susceptible d'être habitée et colonisée d'une manière avantageuse par une popuIation agricole?-Oni, incontestablement. Nous avons des faits en abondance pour établir ce point. Dans presque toutes les parties de ce vaste territoire cle la baie James, on fait de l'agriculture depuis plus d'un siècle, anx différents postes de la compagnie de la baie d'Hudson et le résultat de ces exploitations partielles prouve hors de toute conteste que dans toute cette région le sol et le climat sont aussi favorables à l'agriculture que dans la province de Manitoba et daus la plus grande partie des régioms habitées de la province de Québec.

"J'ai résidé au fort Moose durant quinze ans, dit Gladman. C'est le dépót principal de la compaguie dans le département du sud. Ce poste est tsien à l'abri des vents du nord. Climat et sols bons. La récolte des pommes de terre et d'antres légumes en grande aboudance. L'orge mûrit bien. Des petits fruits, tels que raisins, grosuilles, fraises et frambroisses croissent ì l'état sanyage, en aboudunce. Je ji'ai jamais eu commaissance qu'on ait semé du blé, vu que l'on considérait la saison trop courte. On élerait des bètes à comes, des chevaux, des montous et des cochons qu'on établait durant l'hiver. (Rap. port du comité nommé ponr s'enquérir des droits de la Cie de la baie d'Hudson, pages: 2 et 3 '

"Il y a (au fort iŕose) des pins blanes (les emp"oyés de la corapaguie de: la bale d'Hudson désignent l'épinette blanche sous le nom de pine) mesurant quarante pouces de diamètre...J'ai pénétré jusqu'à 50 ou 60 milles dais les terres et je pense que le sol et le climat de la rivière Moose sont prepres à la production des fèves, des pois et de l'orge, comme dans plusieurs parties du Yorlsshire. J'ai vu de l'orge et de l'avoine récoltées au fort Moose, qui étaient de qualité supérienre à ce que j'ai jamais vu aux ̂les Okney ...J'ai moi-mểme semé daus le mois d'octobre, comme essai, une douzaine de grains cie blé qui après avoir passé l'hivex sous la neige, mûrirent a perfection un mois d'aout. (Témoiguagee du Dr Thompsou devant le comité parleneataire de 1749.)

Robson corrobore ce que Thompson ronstate au sujet do la cultu- 
re du blé. "Du blé d'antomne, dit-il, semé au fort Moose, a bien sup. porté les gélées d'hiver et très bien mûri l'été suivant."

"Ils bèchent (au fort Moose) la terlé, et sèment les pois et les fèves à la mi-mai, et à cette époque ils peuvent bècher jusqu't une profondeur d'un pied et demi ì denx pieds," rapporte Thompson. C'est-àdire que vers le 15 mai, le sol est dégelé jusqu'à un pied et demi ou denx pieds de proforidenr, de sorte que le dégel avance aussi promptement et aussi à boune heure que dans la prorince de Québee. Lit pourtant le fort Moose est un des endroits oì le printemps est le plus froid et lo plns tardif dans toute la région de la baie James. Enfin le capitaine Mitchell constate qu'an fond de la baie, au nord du fort Moose, le dég' al commence le 3 ou le 4 mai.

Tous ces témoignages sont corroborés par celui du Dr Bell, qui a passé plusieurs étés dans cette région depuis 1875. "A notre retour au fort, lisons-nons dans soll rapport pour $1877-8$, page $27 e$,-̀̀ la fin de septembre, nous arons constaté qu'il n'y arait pas gelé do tout l'été ut les plantes les nlus tendres, telles que les melons et les concombres, les fêves, les ci. en plein air, étaient encore rermeilles et florissantes. L'été, tontefois, a probablement été plus beau qu'à l'ordinair

C'est possible; mais il fant avouer qu'on trour d'endroits, dans la province de Québec et dans 1 -.is grande partie de celle d'Ontario, où des pluntes aussi tendres y ne le melon et le palma-christi sont encore vermeilles, n'ont pas été attaqués par la gelé, à cette époque de l'anné.

"Au fort Moose, dit encore le Dr Bell, quoique le sol soit froid, cest une terre glaise humide, avec une surface unie, non égouttée, on récolte chaque année une vuriété considérable de produits de ferme et de jardin. Entre antres produits récoltés en 1874, il y avait 1,700 minots de bonnes pommis de terre. L'avoine, l'orge, les fêres, les pois, les navets, les betteraves, les carottes, les choux, les oignons, les tomates, \&e. sort cultivés at récoltés sans plus de soin qu'il en faut dans les autres parties du Canada, et je suis informé que du blé qui se tronva semé accidentellement a mûri, mais on n'a pas fait d'essais, autant que je sache, pour s'assurer si ce grain pourrait ou non être cultivó régulièrement. On gardo au delà de quatre-vingts bètes à cornes an fort Hoose, sans compter les chevaux, les moutons et les cochons."

(Rapport Geologrique pour 1875-6, page 341.)

Cet ensembie de témoiguages établit d'une manière on ne pent plus positive que la région avoisinant lo föt Moose est un bon pays agricole, jouissant d'un aussi bon climat et possédant un bien meilleur sol que la plus grande partio de la province de Québee. Vependant, lo climat s'améliorera beaucoup lorsque les défrichements auront découvert le sol, l'auront exposé à l'action bienfaisante des rayous salaires et quand les travanx de drainage pour les fins agricoles l'auront débarassé de l'humidité qui furorise le rayomnement nocturne et contribue tant it abaisser la températuro moyenne de l'été, de même qu'à occasionner des gelées à boune heure en autounne. Il est bien 
connu que dans notre province, du moment que les défrichements sont un pen arancés, et le sol ameubli par la culture, on n'a plus de ces gelées a bonne heure qui fout le désespoir des colons lorsqu'ils commencent à défricher leurs terres. A l'ópoque des premiers établissements dans les terrains bas da comté d'Arthabaska, la gelée détruisait une partie d's récoltes; maintenant qu'il y a de grandes étendues en culture, la grelée ne cause ancuns dégats et la ternpérature d'été et d'automne s'est grandement améliorée. Il cu sera de nême daus la région du fort Moose, qui jouit déjà d'une température permettant de eultiver avec succès tons les produits de ferme et de jardin. Nons avons vn aillenrs que la qualité du sol - tue riche terre glaise d'alluvion-est excellente, cu qui, joint à la douceur comparative du climat, fait de cette contrée nne des belles régions agricoles du Canada.

Le climat s'améliore cependant en allant vers le sud, dans le grand plateau égoutté par les rivières qui apportent le tribut de leurs eaux ì la rivière Moose. Sur les bords de ces rivières la culture, même celle du blé, réussit parfaitement.

"J'ai passé l'hiver au fort New-Brunswik (sur la rivière Missinaibi) dit Gladman dans son témoignage, la première année de mon engagement dans le service de ia compagnie, en 1814. A ce poste, le sol est bon. D'excellentes pommes de terre y étaient réeoltées, ainsi que toutes les sortes de légumes. L'aroine mûrissait bien et faisait de bonne fnrine, monlue avec nu nioulin à la main, en acier. L'orge réussissait bien ausisi. A cette époque, en 1814, on n'avait pas essayé le blé ; de puis, ainsi que j'en ai ité informé, l'expérience de cette culture a été faite avec beancoup de snecès. On faisait l'élerage des bètes à eornes, qui étaient gardées à l'étable durant l'hiver. Je ne connais rien qui puisse empêcher de fonder là de bons établisements, c'est un peu éloigué des marchés. Ce poste est environ à mi-chemin entre le fort Moose et le lac Supérieur."

"L'agrieulture etl'horticulture, écrit le Dr Bell, scat pratiquées avec succès par les employés de la compagnie de la baie d'Hudson aux postes des late Mattagamie et Missinaibi. A ce dn.mier endroit M. John McIntyre, maintenant du fort William, m'a informé qu'il a constaté que le blé du printemps mûrit bien. (Rapport G'éologique pour 1875-6 page 340 .)" Disons en passant que dans la région où se trourent ces lacs, les forèts de pins ronges et de pins blances sont communes partout. (Idem, page 341.)

Ces lics sont éloignés d'une couple de cents milles du fort Monse et la région intermédiaire est anssi avantagense, sous le double rapport de la qualité du sol et de la douceur du climat, que les denx points extrê.nes. Quant au sol, voici ce qu'en dit le Dr Bell:

"Dans mon rapport pour 1875, j’ai donné un aperçn général du sol ete de la région située entre les grands lacs et la baie James. La route des canots, depuis Michicopotin jusqu'au fort Moose, traverse des terrains plus ou moins rocheux jusqu'au lae Missinaibi.Cependant, même clans cette région, la proportion de la surfice rochense à l'aire totale sembleêtre comparativement petitc. Mais après avoir passé les "ter- 
rains marécagenx' au nord du lac Missinalbi. le voyageur ne pent pas s'empêcher d'être frappé par l'abondance et la fertilité générale du sol exposé sur les bords des riviéres Missinaibi et Moose, tout lo long de ces rivières, jusqu'an fort Moose. Ce sol se compose en grande partic d'un terroir ou d'une terre brunâtre, un peu graveleuse, reposant su. une glaise d'allurion, et quelquefois sur une couche de glaise stratifiée or sur le roc. solide qui, cependant, se roit rarement, si ce n'est aux chutes et aux principanx rapides. Mais dans le tiers central de cette distance entre le lac Supérieur et la baie Janaes, ou drepuis le fort Brurswick jusqu'au Long-Portage, une glaiso de conleur légère forme ordinairement la surface du sol. En plusieurs endroits, j’ai examinéle terrain jusqu'à une distance d'un mille ou deux de la rivière dans le but spécial de m'assurer de la qualuté dn sol, et dans tous les casjel'ai trouvé exrellent, mass tendant à devenir plus marécagenx en s'éloignant do la rivière, dans la région dévonienne qui se trouve plus bas que le Long Portage. J'ai pris des échantillons du sol en quelques endroits pour les soumettre plus tard à l'analyse. En traversant une région anssi étendue de forêt presque continue, on est porté à oublier la raleur que cette vaste région peut aroir pour les fins agricoles. Mais le spectacle des fermes du fort New-Brunswick et du fort Moose fait voir en petit ce que l'on pourrait étendre à une grande partie de cette contrée. Je ne donte pas qu'il riendra un jour où ce territoire sera habité par une nombrense population:" "Rapport Géologique pou" $1877-8$, pages $7 \mathrm{c}$ et $8 \mathrm{c}$.)

C'est dans cette région centrale que se trouvent les mines de plâtre,de lignite et de fer que nons arous décrites dans lo chapitre consacré à la minéralogie.

Plus à l'est, daus la vallée des rivières Hurricanaw et Abittibi, le sol est d'aussi bonne qualité. Voici ce qu'en dit M. Walter McOuat, qui a fait l'exploration du lac et de la rivière Abittıbi :

"Le lac $\Lambda$ bittibi est onvironné de tous côtés par un terrain de glaise mui. A plusiemrs endroits, couendant, le roc perce la surface de la glaise. Ceci se voit surtout le long de la rive sud du lae d'en hant, où les collines dioritiques, dejà décrites, approchent du lac; mais mème daus ces endroits, il y a généralement une lisière de glaise le long de la rive. Vers le nord, et surtout vers le nord-ouest, la glaise forme la surfice du sol d'une manière presque contiuue et il est bien connu que ce terrain de glaise se continue dans cette direction jusqu'aux rirages de la bain d'Hudson (ce qui comprend ia vallée de la rivière Hurricanaw.)

"Plusiea, acres de ce sol de glaise sont cultivés an poste de la compagnie de la baie d'Hudson à Abittibi, et avec des résultats satisfaisants. Le seu! produit qu'on a récolté jusqu'à présent, e'est la pomme de terre; mais j’ai été iniormé par un homme qui est chargé de la culture (un canadien-franģis qui est depuis plus de trente aus à Abitibbi, mais qui a été élevé daus une fanille de cultivateurs près de Sorel, dans la province de Québec) que plusieurs antres récoltes, y compris le blé, ont été essayéess autrefois, et ont douné des 
résultats tcls, ' qu'il est porţ́ à affirmer que toutes les céréales ordinaires peuvent être cultivées avec autant de fuccès à Abittibbi que sur les bords du Saint-Laurent. Cette opinion, exprimée par un lomme qui a été pendant tant d'aunérs engagé d'une manière pratique dans ia culture du sol, mérite d'ètre signalée et derrait être digne foi.

"On cultire le mais en plus d'un endroit près do la tête du lac T'émiscanningro et l'on dit qu'il réussit bien. To puis persomnellement attester ce fait, vu qu'ou m'en a montré des épis bien mûrs, qui avaient été récoltés durant l'été de 1872 sur la ferme de M. Angus McBride, ì la tête du lac." (Rapport Géologrique pour 1872.73, pages 134 et 135 )

Le lac Abittibi n'est pas à cinquanto milles au nord-ouest du lac Temiscamiugue, ou Témiscumang, comme l'appelle M. McOuat, et il u'est éleré que de 245 pieds an-dessns de ce dernier lac, en sorte que la rempérature d'été doit être à peu près la mème daus les deux localités. Or an lac Témiscamingue, la températuro du printemps, de l'été et de l'antomne est à très peu d'exception près la même que celle de Québec: la moycune est de 37058 pour le printemps, de 65 ○ 25 pour l'été et de $40 \circ 07$ pour l'automne. La température d'été au lac Témiscamingue accuse près de cinq degrés d" chaleur de plus qu'il n'en faut pour la culture du blé, et en supposant que ces cinq degrés manquassent an lac Abitibi, la température serait encore suflisamment élevée ponr permettre de cultiver dans cette localité toutes les céréales, même le bié, ce qu est l'avis de M. McOuat et de l'agriculteur dont il cite l'op:nion.

La qualité clu sol de la valée de la rivière Hurricanaw est connue : on sait qu'elle est excellente, puisque la surface dn terrain se compose ue terve glnise et de dépôts alluriens; mais il n'y a pas de données précises star la nature du elimat. Cependant, il est faeile de s'en former une juste iảée en procédaut par induction. Si la température d'été, dans les localités situées plus an nord, est propre aux opérations agricoles, il est clair qu'nlle doit l'être pour le moins autant dans la coutrée de la rivière Hurricanaw, plus an snd. Or voiei co que dit le Dr Bell de la température au lort Rupert et de tonte la région aroisinant l'extrémité nord-est de ia baie James :

"Durant notre voyage le long de la cóte (jusqu'au golfe Richmond, à plus de 500 milles au nord du fort liupert), en allant et eu revenant, durant les mois de juillet, août et septembre, nous avous joui la pius grande partie du temps d'une très belle température. I' est tombé très peu de pluie et il n'y a eu que deux ou trois jours de brouillard. Les vents les plus fréquents renaient du sud et la température était chande et agréable. La supériorité de la température, comparée à celle du lac Supérieur,a été un sujet de remarques fréquentes parmi nos " voyageurs," qui avaient été habitués pendant toute leur vie au clirnat du lac Supérieur...

"D'après le tableau des observations qui précède, la température moyenne de l'a: entre le 11 juillet et le 21 septembre serait de $62 \frac{1}{2}$, ce 
ani est à très peu de chose près la température moyenue des trois mois et la moyenno pour juillet et nout serait de $65 \frac{1}{2}$. Comme le plus grand nombre de ces observations ont été faites lo matin on le soir, et comrac les nuits étaient généralement chaudes, grâce à la prédominence des vents du sud, ce chiffre n'est pent-être pas éloigné de la vraie température moyeune de ees deux mois et il est seulement do ho au-dessus de la moyeme de la température, pour les mêmes nois: des dix principales stations (météorologiques) entre Halifax, daus la Nouvelle-licosse, et le fort Simpson, sur la rivière Mackenzie." (Rupport Géologiqne pour 1877-8, pages 26c et $27 \mathrm{c}$.

Comme nous l'avons observé plus haut, la température, dans la vallée de la rivière Hurricanaw, doit être pour lo moins aussi élevée que celle de l'East-Main, qui est bien plus au nord, et comme la tompérature d'été de cette dernière région est de $65 \frac{1}{2} \circ$, il faut bien en conclure que celle de la contrée qui nous ocenpe est pour ls moins anssi élerée, ce qui est plus que suffisant pour la culture de toutes les céréales, mème du blé.

Le grand territoire égontté par les rivières Hurricanaw et Abittibi fuit doac incontestablement partie des régions cultivables au double point de vue $d u$ sol et du climat. Ce territoire a presque la même étendue que la supł'ficie du Nouvean-Brmnswick, qui est habité par plus de 300,000 ârnes.

Dans la région de la rivière Albany, qui est à pen près égale à l'Ontaonais par la longneur de son cours et l'étendua de bon terrain qu'elle égontte, les eriployés de la compagnie de la baie d'Hudson font depuis longtemps de l'agriculture avec un bon succes et récoltent des arains de toutes sortes, mẹme du blé. Le sol et le climat sont presque partout très propres à l'agriculture. Ce fait est attesté par un grand nombre de témoignages, dont nous citons les principanx. l'renous d'abord ce que Dobbs,page 13,écrit sur le climat.Après avoir dit qu'il est à peu près le même qu'au fort Noose et au fort Albany, il ajoute en parlant des saisons à cetto dernière localité :

"En 1729, la gelée commença en octobre... Sur le ruisseau, près du fort, la glace se forma le 13 ; le 21 il y avait beancoup de glaces flottantes sur la rivière. Ise 31 elle était prise jusqu'au ruisseau Charles. Le $\tilde{5}$ novembre, la glace était prise sur toute la rivière, mais cette glace n'était pas assez forte pour porter (un homme). Mars.. jusqn'an 17, temps beau, clair, avec un peu de neig'e De cette date au 29 , temps clair, joliment chaud. Le 30 , tombée de neigre, et alors il commença à dégeler sur le haut du jour. Ce dégel continua jusqu'à la mi-arril ; à cette date, deux joư's de gelée... le 29, la glace partit jusqu'à la tête de l'tle... la rivière continua à charrier des glaces jusqu'au 5 mai... Alors l'eau de la rivière baissa de cinq pieds, en conséquence de la rupture de la glace dans la mer. Le 8 les Sauvages arrivèrent en canots pour faire la traite. Le 16 on commença à bêcher le jardin. Le 22 le cours de la rivière devint régulier... temps beau, chand, du 11 mai à la mi-septembre...

"Très gros arbres de toutes sortes (pages 15-6)...il y a énormément 
de bonne herbo pour faire du foin... et l'on peut récolter partout dans l'intérienr' des terrís toutes sortes de plantes léguminouses et de grains et cultiver toutes sortes d'arbres fruitiers do mène que sons les mêmes climats en Énrope, car tout ce qu'on a essayé de eultiver a très bien réussi."

Tout cela est confirmó par les témoignages entondus par le comité elu parlement impérial en 17 i9. "J'ai mangé là des navets, dit Sargent, aussi bons que cenx que j’aie jamais mangés en Angleterro... A mon avis, l'avoine mùrirait an fort Albany, où j'avais un,cerisier qui produisait des cerises noires...Je crois que la belle saison est suffisarnineut longue pour lạ culture du blé... L'herbe pousse en quantité suffisante pour nourrir le bétail...Jo crois que le grain viendrait à maturité à vingt milles au,nord du fort Albuny." Griffin a va l'avoine renir à perfection à ce jort. Il y a vu récolter des navets, de la laitue des choux et quelques carottes. Les pois, les fèves et les choux vionnent en abc.1dance et à pərfection. "Si, dit-il, j'obtenais la concession de cent acres de terrain et la liberté de fairo la traite, jo m'établirais là du plus graud cœur." Enfin le climat et le sol sont de même qualité qu'au fort Moose, où il est bien connu que l'ngriculture rénssit parfiaiteñent.

Lo climat et le sol s'améilorent sensiblement à mesure qu'on avance vers le sud et lo sud-onest, et dans la région supérieure do la rivière on cultive tontes les céréales aux environs des postes de la compaguie de la baie d'Hudson, notamment aux forts Osnaburg. Henley, Martin's Fall et du lae Seul. Barnston, qui a résidé à Martin's Falls, à 250 milles de l'embouchure de la rivière, prétend qu'en hiver cette localité a la température de la Russie, et durant les mois de juillet et et d'août celle de la France et de l'Allemaugne. Dans les stisons ordinaires, les bourgeons se forment sur les arbres vers le 12 mai et les feuilles ont tout leur développement à fa fin de ce mois. Elles jaunissent et commencent à tomber a commencement d'octobre. Il gèle quelquefois dans la première quinzaine de juin. La neige commence ì tomber le 20 octobre ot convre le sol le 1er novembre. En 1871 , le Dr Bell a contstaté que sur les bords du lac Long, les feuilles des pommes de terre n'avaient pas encore été attaquées par la gelée lors. qu'on les récolta daus la première semaine d'octobre.

Ces données établissent elairement que le cours des saisons est à péa près le même dans la vallée de la rivière Albany que dans la plus grande partie les antres provinces du Canada. Ita végétation commence aussitôt le printemps et la gelée aussi tard l'automne. Entre Martin's Falls et la baie James, la rivière est libve de glace duraut six mois et dans l'été la température est suffisaminent élevée pour faire mûrir toutes les céréales. Le climat est semblable dans la partie sud de la vallée de la rivière Serern, que lobbs représente conme une très belle rivière, coulaut à travers une région bien boisée, riche et fertile.

Si maigres, si incomplètes qu'ils sont, comparativement à l'immeuse étendue du territoire auquel ils s'appliquent, ces renseignements éta- 
blissent d'une manière incontestable que cette grande plaine est réellement propre à la colonisation. Du moment qu'on l'aura dépouillée des belles forêts de pins et d'épinettes qui la recouvrent, la terre rétribuera libéralement lo travail du défrichenr qui la livrera do la eulture. Les produits de la ferme tronveront, dans les premiers temps du moins, un écoulemont facile dans les chantiers, car du moment qu'on en connattra la valeur et l'étendue, on ne manquera pas de livrer a l'industrie forestière l's bois qui se rencontrent presque partout en quantité et de qualité snffisantes pour fournir un fort contingent an commerce d'exportation. Les rivières offrent de grandes facilités et bien peu d'obstacles sérieux à la flottaison des bois : ces facilités sont cornplótées par les ports de mer de la baie James, qui sont moins éloignés que Québec de Liverpool et des autres grands centres de commerce maritime du lioyaume-Uni. L'exploitation des mines contribuera aussi au développement de ce pays, qui constitue uue des parties les plus intéressintes, les plus riches du Canada, une des contrées qui ont un bel avenir. Nons avons vu que l'étendue des terres arables, susceptibles d'une exploitation agricole réellement avantageuse,est d'à peu près $108,800,000$ acres carrés. C'est un peu plus que l'étendue totale de la Suède, qui est habitée par une population de 4,500,000 àmes. Il n'est pas irraisonnable de prédire que la région de la baie James pourrait faire vivre une population aussi nombreuse. Le climat de ces deux pays est le même, ils sont également riches en mines et en forêts et il $n^{\prime} y$ a de différence qug aans le sol, qui est bien meilleur daus li région de la baie James qu'en Suède. Enfin, le fort Moose n'est pas beaucoup plus isolé qua Stockolm, du este du monde; il est pareillement accessible par la navigution, er il sera beaucoup plus facile de le mettre, an moyen d'un chomin de fer, en communication directe arec toutes les grandes villes commerciales des anciennes provinces do la Coufédération, qu'il u'est facile de relier par ce moyen au reste de l'Europe, la

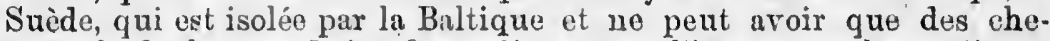
mins de fer locaux. Qu'on fasse disparattre l'ignorance, les préjugés et l'apathie cui existent au sujet du territoire do la baie James, et avant vingt ans il y aura là une province riche et prospère, avec ses lignes de navigation, ses chemins de fer, ses grands centres do commerce, enfin tout ce qui constitue le progrès et le bien-être d'un peuple. 


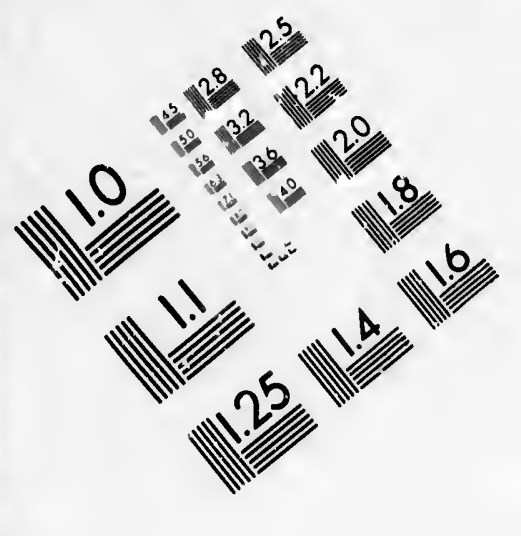

\section{IMAGE EVALUATION TEST TARGET (MT-3)}
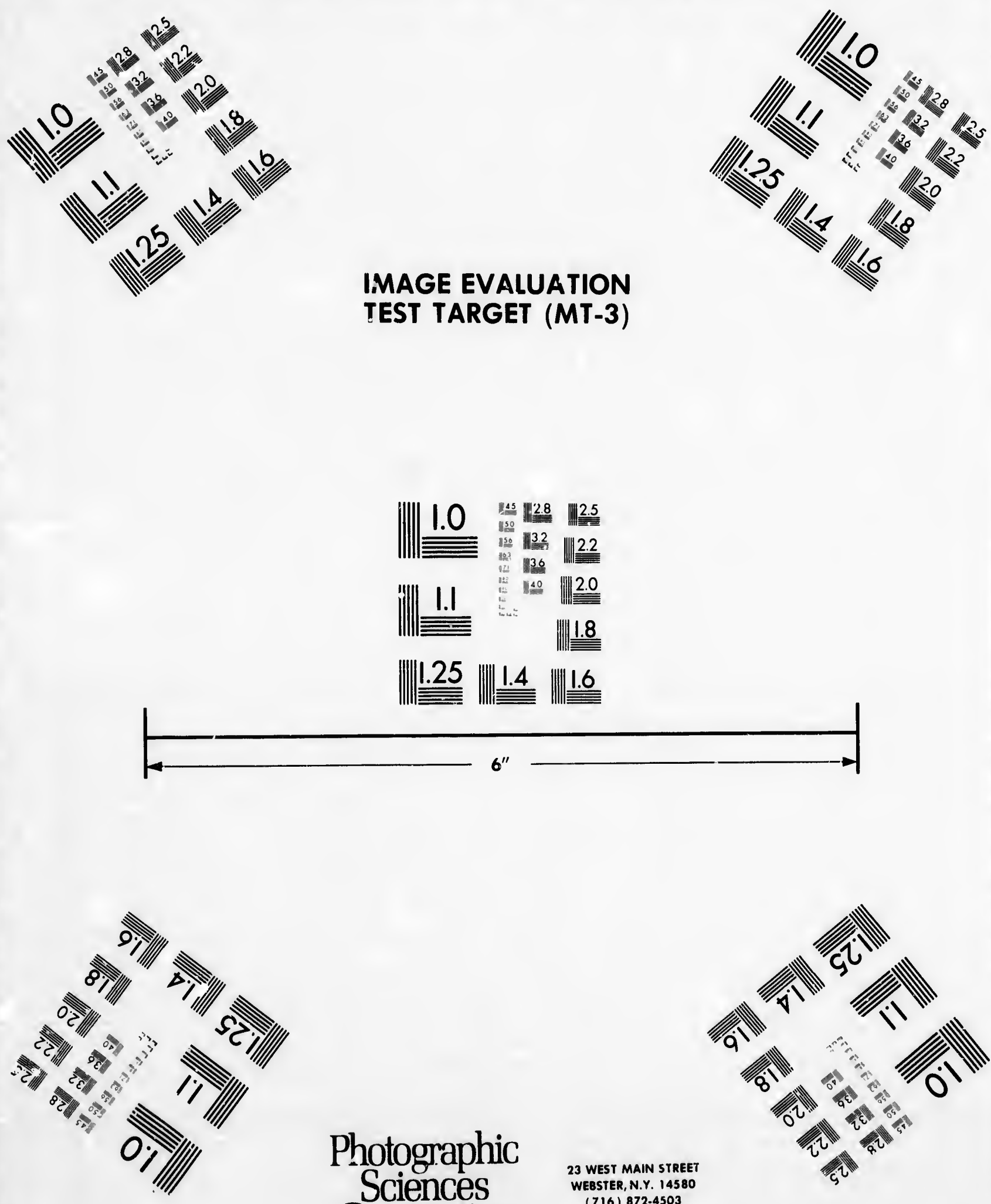

Photographic Sciences

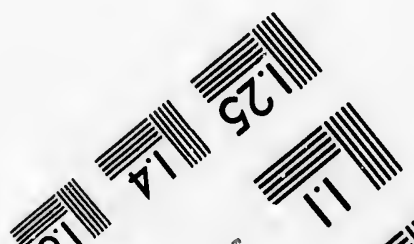

Corporation

(716) 872-4503 



\section{OHAPITRE VIII}

NECESSITE D'UN CHEMIN DE FER POUR OUVRIR CE TERRITOIRE $\boldsymbol{A}$ IAA COLONISATION-QUELLE DIREOTION DOIT SUIVRE OE CHEMIN-DANS QUELLES CONDITIONS IL PEUT ETRE CONSTRUIT-L'AVENIR QUI IUI BST RESERVE

Ponr coloniser, il faut des chemins de fer ; en vain une région offrirait-elle les plus grands avantages sons tous les autres rapports, elle n'est pas recherchée si elle n'est mise en communication avec les grands centres d'affaires par une voie ferrée. Depuis une quinzaine d'années, des milliers de jeunes gens voudraient aller s'établir dans la belle vallé du lac St-Jean, mais en sont empèchés par l'isolement fans lequel se tronve ce beau pays. Au nord-ouest, les plus beaux terrains agricoles se trouvent dans la régicn de la rivière à la Paix et de l'Athabaska, qui est bien supérieure comme sol et comme climat a celles de la Saskatchewan et de la Rivière-Rouge; meis ces dernières régions sout traversées par un chemin de fer, et cette considération seule les fait rechercher de préférence aux beaux pays que traversent l'Athabaska et la rivière la Paix, où le climat est plus doux, le sol meilleur, où les richesses minérales de toutes sortes abondent, mais ou il n'y a pas de perspective qu'oll constrliso d'ici à loug. temps un chemin de fer pour relier cetto magnifique contréo au reste du Nord-Ouest.

Cette nécessité d'un chemin de fer, qui peut être relative ailleurs, est absolue pour le territoire de la baie James. La navigation de la baie et du détroit d'Hudson peut donner au commerce des transports une certaine accommodation pendant trois ou quatre mois dans l'année; ma: cela n'est pas suffisant pour transporter les millions de minots de grain et les énormes quantités de bois que cette contrée pourra fournir à l'exportation, quand elle aura une population assez nombreuse. D'ailleurs cette comunication par la mer ne serait pas coustante, et du moment que la navigation cesserait, ce pays se trouverait complètement isolé. Enfin, pour le transport du commerce ordinaire, il faut des moyens de communication prompte, peu dispondieux, susceptiblss d'être utilisés tous les les jcurs et d'une manière permanente, ce qui ne peut se faire que par nue voie ferrée. C'est ce qui expliciue pourquoi la colonisation progresse si rapide. ment lorsqu'elle est favorisée par ces grandes artères du commerce moderne. Tant que la construction du chemin de fer Canadien du Pacifique n'a pas été assurée d'une manière positive, il était presqu'impossible de faire prendre aux immigrés la route du Nord-Ouest; mais dès qu'il fut connu que ce chemin se ferait, le désir de prencire des terres dans la région de rivière Rouge et de la Saskatehewan s'est em. paré de tout le monde, méme de la population bourgeoise des anciennes provinces, dort l'émigration au Nord-Ouest commence à prendre des proportious alarmantes. Des terrains qui ne valaient rien il y a quatre ou cinq ans se vendent aujourd'hui d dos prix exorbitants, et 
Winnepeg, qui nétait qu'une humble bourgade en 1870, est en train de devenir promptement le Chicago du Canada. Il en sera de mème dans la superbe région de la baie James, du moment qu'on prendra les moyens de la rolier aux autres provincęs par un chemin do fer.

La construction de ce chomin de fer pourrait s'exécuter dans les conditions les plus faciles. La nature a marqué elle-méme le tracé que cette voie devrait suivre. Au sud-est de la baie James, les Laurentides, qui entourent le bassin formé par cette baie, n'ont que peu d'élévation, ne sont pas même assez hautes pour mériter lo nom de montagnes. Leur sommet forme un plateau uni et s'abaissant en pente douce des deux côtés. L'uniformité de niveau se prolonge sur Ses deux versants, au nord-ouests jusque dans la vallée de la rivière Moose, et an sud-est jusque dans celle du lac Saint-Jean. A partir de ce deruier point le tracé adopté pour is gêmin de fer de Québec au lac Saint-Jean ne présente aucune difficuî : iause jusqn'à Québec. Pour rendre l'exposé de ce projet plus clair, entrons dans quelques détails topographiques.

$1^{\text {ro }}$ Section - En partant de Québec, le chemin de fer du NordOuest - nous lui donnons ce nom par anticipation - suivrait jusqu'à l'extrémité sud-ouest du lac Sain--Jean le tracé de la ligne actuellement en voie de construction, e'est-à-dire que cette ligne deviendrait le premier tronçon du chemin projeté. Ce tronçon aurait environ cent quatre-vingts milles de longueur.

$2^{\circ}$ Section - Cette deuxième section șrrcit formée par l'espace compris entre lo lac Saint-Jean et la hauteur des terres, ou une distance d'environ cent trente milles. La ligne passerait au sud du lac Askatiche, sur les confins de la hauteur des terres, qui est élevée de 1288 pieds au-dessus du nivear de la ızer.

$3^{\circ}$ Sectıon - Elle s'étendrait do la hauteur des terres à la rivière Mattagrami, à cent milles au sud du fort Moose. C'est l'endroit où la Mattagami cesse d'être navigable dans les haul es taux. La longueur de cette dernière section serait d'environ 320 milles et la déclivité d'une douzaine de cents pieds.

La longuear collective des trois sections serait d'environ 630 milles.

Le tracé de la première section et la nature des terrains qu'ello traverse sont trop bien connus pour qu'il soit nécessaire de donner des détails sur ce point. Les rapports de MM. les arpenteurs Casgrain et Sullivan, puis celui de M. l'arpenteur Dumais sur la région situóe entre le lac Saint-Jean et le lac Edouard, établissent qu'en plusieurs endroits et sur de longues distances, le tracé adopté iraverse des terres susceptibles do culture, des forêts d'une grande valeur. Quant as $x$ accidents du terrain, ils n'offrent aucun obstacle sérieux à la construction d'un chemin de fer. On sait que cette ligne a pour but. de mettre la vallée du lac Saint-Jean en communication directe avec la ville de Québec.

La deuxième section traverserait une région généralement unie, très-fertile sur nne grande partie de ce parcours. La surface du sol 
est quelquefois accidentée par des rochers qui la percent; mais cos rochers n'ont que très peu d'étendue et sont de rare occurrenoo : ils ne forment nulle part le moindre obstacle d l'établissement d'une voie ferrée, puisqu'ils pẹvent être évités avec la plus grande facilité, sanş affecter sensiblement la course générale de la ligne. Il n'y aurait pas de rampes à fortes pentes; le terrain est ani partout et descend graduellement, insensiblement depuis la hauteur des terres jusqu'au lac Saint-Jean. La différence de niveau entre ces deux points n'est que de 978 pieds : le lac Askatiche, sur. la hauteur des terres, est d 1288, et le lac Saint-Jean à 310 pieds au dessus du niveau de la mer. Cette différence de niveav donne une inclinaison moyenne de 7.52 pieds au mille.

La ligue passerait une certaine distance - de vingt a trente milles - au sud de la rivière; mais, comme les reliefs du sol sont a peu près les mêmes a cette distance que sur les bords de la Chamouchouan, il est facile de voir qu'elle ne rencontrerait nulle part d'élévations abruptes et difficiles à franchir par un chemin de fer. De son embouchure au trente-sixième mille, la Chamouchouan coule à travers une région comparativement unie et son cours sélève de 219 pieds an-dessus du lac Saint-Jean; mais a partir du trente-sixiène mille, ses rives deviennent rocheuses, souvent escarpćes et encaissées dans des collines de gniess dont la hauteur varfe de cent cinquante à cinq cents pieds. Ces collines ne s'étundent pas loin daus l'intérieur des terres. Au confluent de la riviëre Shecobish, vers le milieu du ciuquante huitième mille, le cours de la rivière est à cinq cent soixante pieds au-dessus des eaux du lac, ce qui fait une pente de trois cent quarante et un pieds pour les vingt-deux milles compris entre ce dernicr endroit et le trente-sixième mille. A cux seuls, les rapides et les chutes de la Chaudière, qui n'ont pas un mille de longueur, abaissent le cours de la rivière de cent ving i et un pieds. Entre !a rivière Shicobisk et le ruisseau de la Cloche, distance de ringt-trois milles, la pente du courant est de quatre-vingt-six pieds. De la rivière du Chef au lac Ashuapmouchouan, la rivièro n'est qu'un rapide continuel et fait une descente de cent quinze pieds entre le $102^{\circ}$ et le $99^{\circ}$ milles, et de coixante-cinq pieds dans les neuf milles suivants. La pente $d u$ courant diminue graduellement depuis ce dernier endroit jusqu'aux sources de la rivière. Dans toute cette section, il n'y a donc que des pentes doucces, des rampes très ordinaires sur tous les chemins de fer, si l'on peut juger du niveau du sol par celui du cours de la rivière.

Une bonne partie de ce tracé passerait à travers des terrains d'une richesse et d'une fertilité extraordinaires. A partir de l'estrémité sud-est du lac Saint-Jean et jusqu'd une soixantaine de milles en allant vers l'ouest, le sol est excellent; il se compose d'une terre forte, le plus souvent d'une couche de cerre jauno grasse reposant sur la glaise. Oes terrains sont unis et exempts de rochers. La forêt se compose de merisier, d'orme, de frêne, de tremble, d'épinettes, de 
sapins, de pins, eto., et tous ces arbres sont de fortes dimensions." Ces berux terrains se continuent rers le sud jusqu'au SaintMaurice et ils renferment plus de 8,000,000 d'acrcs d'ex sellente terre arabl. . Cette étendue est suffisante pour étzblir ane population de trois à quatre cent mille âmes. Il ne faut pas être prophìte pour prédire que le chemin de fer qui mettra cette région en comniunication avec Québec a la plus bello perspective de succès comme entreprise payante.

La troiême section serait facile d̀ construire et traverserait upe immense étendue de bons terrains. Entre la hauteur des terres et le terminus sur la rivière Mattagami, la différence de niveau est d'enriron 1188 pieds, ce qui fait une moyenne de 3.71 pieds au mille. Il n'y anrait pas de rampes abruptes dans tont ce parcours. Du moment qu'on a passé la hauteur des terres, le sol est plan et la surface du pays incline insensiblement et uniformément en allant vers l'ouest jusqu'à la baie James. Le cours des rivières Nottaway et Hurricanaw qui suit à pen près la même direction que le tracé de la ligne projetée est à peine accidenté par des cascades insignifiantes, vers la ligne de démarcation entre les terrains laurentiens et les terrains siluviens; mais mème dans ces endroits, la surface du sol est unie, nulleznent percée par des éruptions rocheuses d'une étendue qui vaille la peine d'être mentionnée. Sur plus des deux tiers de cette distance, les terrains sont formés par des glaises d'alluvicn el des débris de calcaire qui constituent un sol d'une excellente qualité. On pourrait faire presque partout de bons établissements agricoles.

Quant au trafic qui se ferait sur cotte section, il est facilo d'en apprécier l'importance quand on considère que cette ligne aurait son terminus à peu près dans le centre d'un territoire renfermunt plus de $100,000,000$ d'acres be bonne terre arable, des forêts inépuisables, des mines de lignite d'une valeur incontestable et du bon minerai de fer en quantité presqu'iliimitée et des pêcheries d'une grande richesse. On pourrait anssi prendre le fort Moose pour terminus; mais cela éloignerait de cent milles du chemin de fer la pius belle partio de toute cette région, tandis qu'en fixant le terminus à la tète de la navgation sur la rivière Moose, on n'isole la partie nord que durant l'hiver, vu qu'en été elle aurait la navigation pour communiquer avec le chemin de fer.

Aux yeax de beancoup de gens, l'idée de construire un chemin pour aller à la baie James semblera peut-être extraordinaine, sinon absurde ; on est si habitué à n'entendro parler de cette région que comme un pays inhabitable, aride, sans ressources aucunes, que beaucoup de gens, sans se douner la peine de rélléchir et de se renseiguer, seront portés à se demander s'il ne faudrait pas autant parler d'un chemin de fer pour aller au pôle nord. C'est le raisonnement de l'ignorance et des préjugés. Cependant, il ne faut pas réfléchir bien

- Rapport de 11. l'arpenteur Gagnon. 
longtemps pour se convaincre qu'il est peu d'eutreprises de chemins de fer dont l'utilité sjit plus évidente et les perspectives de succès plus brillantes. Il y a dans la région de la baie James plus de cent millions de bonnes terres cultivables sous le double rapport du sol et du climat et cette région ne pent être ouverte a la colonisation que par la construction d'an chemin de fer,puisque la navigation par la baie d'Hudson ne peat donner qu'un moyen de communication tout à fait précaire, et dans tous les cas bien insuffisaut. Comme nous l'avons dit ailleurs, l'étenduo des terres cultivables dans la région de la baie James égale l'étendue totale du la Suède qui, bien que situéu. dans les mèmes conditious sous tous les rapports, n'en est pas moins habitée par une populatiou de 4,000,000. Pour ue pas heurter de front les préjugés, nous admettrons que notre Suède Canadienne ne sera jamais habitée par plus d'un million de personnes. Mais; cela n'est-il pas suffisant pour assurer l'aveuir d'un chemin de fer et en motiver la construction? Avons-nous beancoup de lignes, dans les anciennes provinces do la Confédération, yui aient été établies dens des nonditions aussi arantageuses? Un inillion! mais c'est presque le chiffre de la population de la province de Québec. On objectera peut-être que cette région n'est pas habitée-Saus doute qu'elle ne l'est pas, et ¿'est précisément à cause de cela qu'il faudrait un chomin de fer pour l'ouvrir a la colonisation. Les plaines de la Saskat. chewan ne sont pas plus habitées, ce qui n'empêche pas qu'on est en voie d'y construire neuf cents milles de chemin de fer. Si la construction de ce chemin de fer a sa raison d'être, celle du chemin de la baie James l'a pareillement, puisqu'aux environs de cette baie il y a une pius graude étendue de bonnes terres cultivables qu'il n'y en a dans touts la vallée de la Saskatchewan.

\section{CONCLUSIONS}

En résumant tout ce qui précède, on arrive uécessairement aux conclusious suivantes :

$1^{\circ}$ Le bassin méridional de la baie d'Hudson, - c'est-d-dire la partie des territoires cédés au Canada par la Compagnie de la baie d'Hudson, bornée au nord par le $60^{\circ}$ degré de latitude, à l'est par le $70^{\circ}$ méridien de Greenwich, au sud par la ligne de falte des Laurentides et a l'ouest par une ligne traversant diagoualement le $110^{\circ}$ degré de longitudé, du sud-ouest au nord-est - forme une aire d'environ 750,000 milles carrés, ou plus de trois fois l'étendue de la prorince de Québec. De cette étendue, il faut déduire environ 250,000 milles pour l'espace occupé par les eaux de la partie sud de la baie d'Huảson et de la baie James, ce qui laisse poux les terres.nne superficie de 500,000 milles, ou plus de deux fois l'étendue de la province de Québec. 
$2^{\circ}$ La configuration de ce territoire ressemble à une section latérale de cóne tronqué ou à un are dy cercle dont les Laurentides forment la circonférence, la partie sud de la baie d'Hudson et la baie Jumes le centre, puis les grandes rivières qui f e jettent dans les deux baies, les rayons. La surface de cette immense contree est partout uniforme, a peine accidentée par de très rares buttes rocheuses de très peu d'étendue et d'à peine deux ou trois cents pieds de hauteur, dans les terrains d'origine cristalline qui avoisinent le sommet des Laurentides, qri ne s'élèvent guère à plus de mille pieds au-dessus da niveau de la mer, au sud de la baie James, et s'abaissent graduellement en allant vers le nord-ouest. Entre les Laurentides et la mer, le niveau du sol s'abaisse insensiblement, à tel point qu'on trouve \& peine deux r $:$ trois chutes d'une cinquantaine de pieds de hauteur, vers le point ile roncoutre des terrains cristallins avec les formations plus molles de l'âg? silurien, sur le cours de 'toutes les grandes rivières qui traversent cette région pour arriver à la baie James ou à la baie d'Hudson.

$3^{\circ}$ Les terrains un peu éiavés, formant une étroite rivière qui borde tout ce territoire au sud-est, au sud et au sud-onest, appartiennent aux formations laurentiennes et huroniennes : ils sont plus accidentés, plus socs, que ceux de la zone iuférieure, qui appartiennent aux diffé ents âgea du groupe silurien. Dans cette zone, le sol est marécageux et humide en certains endroits, parce qu'il est trop uni ; mais il se compose presque partout d'argile, en strates on en dépòts alluviens, de terre végétale ei de dépôts superficiels qui constituent d'excellents terrains agricoles. Les lacs sont comparativenient très-rares, ce qui n'empecche pas qu'on peut partout se procurer facilement et en quantité inépuisable de la bonne eau potable en creusunt la terre à quelques pieds dé profondeur.

4. Ce raste territoire renferme au moins 244,600 milles carrés, on $156,544,000$ arres de bonnes terics agricoles, susceptibles du plus haut degré de culłure au double point de vue du sol et du climat, qui sont aussi bons, sinon meilleurs, que le sol et le climat de la "zone fertile" de la Saskatchewan. Ces terres cultivables se répartisssut de la manière suivante dans chacune des trois divisions naturelles de ce territoire : région de l'est, ou East-Main, 1000 milles, ou 640,000 acres ; région du sud, on de la baie James, 170,000 milles, ou 108,800,000 acres; région de l'ouest, on des rivières Churchill et Nelson, 73,600 milles, ou $\$ 7,104,000$ acres. En supposant que ces régions s'établissent, quant à l'étendne movenne cultivée et occupée par chaque habitant, dans la même proportion que la province de Québec, elle renferme assez d'espace et de bounes terres pour supporter et faire virre dans l'aisance une population de $15,(00,000$, quant à l'étendue occupée, et de $31,308,000$, quant à l'étendue en culture. En se basant sur les mèmes données, prises dans le recensement de 1871 pour ce qui concerne la province de Québec, chacune des trois régions du territoire que uous étudions pourrait supporter la populatiou euivante : 
Région de l'East-Main...... $\quad 64,000$ ames......

Région de la baie James... 10,880,0C0 " .......

Région des rivières Chur-

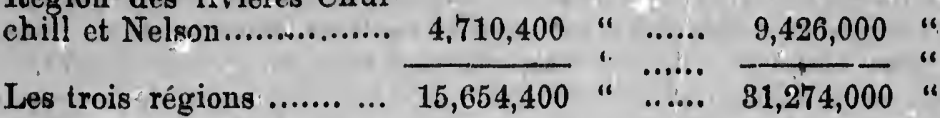

La colonne de ganche est basée sur l'étendue occupée, et celle de droite sur l'étendue en culture dans la province de Québec en 1871.

5 - L'étendue des forêts où les bois sont susceptibles d'étre employés pour les usages domestiques et pour l'exportation forme une aire d'an mois 200,000,000 d'acres. Jusqu't la rivière George ou Grande-Rivière, dans la région de l'East-Main et jusqu'd nne cinquante de milles de la mer, sur les bords de la rivière Nelson et de la rivière Hayes, on trouve des forêts dont les arbres sont assez grus pour faire des bons billots de vingt-quatre pouces de diamêtre, notamment les épinettes blanches. Ies principales essences sont lo pin blanc, le pin rouge, l'épinette ronge, le cyprès ou pin de Banks, le tremble, le peuplier, le sapin, le cèdre et le bouleau. L'épinette blanche est le bois le plus répandu et le plus précieux : dans les trois régions elle forme des forêts bien supérieures, quant a leur étendue et à la grosseur générale des urbres, aux forêts de même espèce que nous arons dans la province de Québec. L'épinette rouge forme des forêts presqu'aussi belles dans la contrée située ai sud de la baie James. Il $\mathrm{y}$ a de belles forêts de pin blanc et des forêts de pin rouge de moindre étendue, dans la lisière qui avoisine la hauteur des terres entre le lac Abittibi et les sources de la rivière Kenogami. O'est la continuation des forêts du haut de l'Outaonais. On rencontre aussi dars cette région des sapinz et des cèdres suffisamment longs et gros pour avoir de la valeur commo bois de service. 'Le boulean est très gros dans les environs du fort Norway, où les Sauvages l'entaillent le printemps pour faire du sirop avec sa sève, comme on fait du sucre avec la sève de l'érable dans d'autres parties du Canada Le tremble et le peuplier sont les essences dominantes dans la valiée de la rivière aux Castors et du lac Vert où ces arbres atteignent des dimensions colossales et forment de bellos forêts.

L'exploitation de ces forêts, pour le commerce d'exportation, pourrait se faire dans les conditions les plus avantagenses. Les grandes étendues de terrain qu'elles couvrent, dans l'East-Main et la région de la baie James, sont traversées par de nombreuses et grandes rivières dont le cours est généralement libre et nulle part intercepté par des obstacles sérieux. Sur la plupart de ces rivières, la flottaison des bois peut se faire avec la plus grande facilité, dopuis la hauteur des terres jusqu'à la mer, ce qui fait une distance d'environ trois cents milles, à partir des endroits les plus éloignés. Puis, des ports les plus reculés de la baie James, la distance jusqu'aux ports d'Angleterre n'est pas plus grande, est même moindre qu'eutre Québec et Liverpool, et guère plus considérnble qu'entro les principaux ports 
du Royaume-Uni et ceux de la Baltique, où sont pris pour la plus grande partie les bois qui sont importés en Angleterre.

$6^{\circ}$ Il y a daus cette région des mines d'une puissance et d'une richesse incalculables. Les principaux minéraux sont lo fer, le plomb le cuivre, le manganèse, l'argent, le lignite, l'authracite, le plâtre, le pétrole et différentes espèces de pierres d'oruementation. Les espèces les plus susceptibles d'exploitation sont le for. le plomb, lo lignite, lo nuanganèse et le plâtre.

Il y a des gisements considérables d'oxyde de fer magnétique, sur sur les bords de la rivière Mattagani; d'hématite rouge, ou 'de for oligiste, dans les ccuuches diluviennes de la rivière Albany ; d'hématite brune, ou de limonite, au grand rapide de la riviére Mattagami. Ce minerai a donné à l'analyse 52.42 pour 100 de fer métallique. Mais ces mines ne sunt rien, comparées à celles des lles du détroit de Nastakopa, où l'on a découvert du minorai de fer spatique manganésifêre en quantités inépuisables. Dans toutes ces 1les, qui forment une chalne de plus de quatre-vingt-dix milles de longueur, le minerai de fer forme a la surface une épaisseur d'une vingtaine de pieds Deux échantillons ‘de ce min rai ont douné respectivement à l'analyse 25.44 . et 27.83 pour 100 de fer métallique, ce qni constitue un minerai d'une exploitation lucrative, puisque d'après Osborne, qui fait autorité en pareille matière, tout minerai de fer renfermant au moins 16 pour 100 de métal peut '3tre exploité avec profit $:$ Le minerai de Nastakopa contient presque deux fois cette proportion - de fer inétallique. Outre cette qualité, son extraction pout se faire dans des conditions tout particulièrement avantageuses. Le minerai forme la couche supérieure da terrain, qui est complètement dénudé; la roche, comme dit le Dr. Bcll, s'est. fracturée par l'action de l'air et de la gelée, en sorte qu'on peut enleve: une grande partie du minerai saus être obligé de miner, ce qui diminue énormément løs frais d'extraction et en réduit le coût, sorti de la mine et prêt à livrer à l'exploitation ou aux hauts fourneaux, à une somme insignifiante. Et dans les environs des fles il y a partout d'excellents mouillages où les navires,même ceux d'un fort tirant d'eau, peurent aborder et ancrer avec la plus grande sécurité comme la plus grande facilité. Ces gisements sont assez riches et assez étendus pour fournir au moins 40 , 900.000 de touneaux de fer.

Ce minerai est à peu près sans égal pour la fabrication de certains aciers, notamment l'acier Bessmer, dont l'usage s'est tant répandu depuis quelques années, surtont pour la confeetion de lisses de chemin de fer. En Angleterre et aux Ltats-Unis, a Troy principalement, une bonne partio des fabriques d'ncier Bessmer est alimentée par la fonte des minerais de fer spatique qu'on importe d'Allemagne en grande quantité. Aux Etats-Unis, on a ouvert depuis quelques années treize usines où l'on fabrique l'acier Bessmer pour le convertir en lisses de chemins de fer, tant la demande de ces lisses augmente rapidement.

- Osborne, Metallurgy of Iron and steel, page 31. 
Oes usines emploient 10,840 ouvriers, dont les salaires se isont élevés a $\$ 4,980,839$ en 1881. Pour la mème année, le produit de cess usines a été de $\$ 55,835,000$, et les dépenses pour matières premières réparations, etc., ont atteint le chiffre de $\$ 36,375,926$. Le enpital engagé dans ces treire établissements est de $\$ 21,000,000$. Lin France, il y a plus de trenie-cing usines où l'on transforme le minerai de fer spatique en fonte blanche à grandes lames et on acier. Enfin, en Allomagne, c'est avec ce mème minerai, mais généralement de qualité inférieure à celui de Nastakopa, qu'on fabrique les fameux aciers de Styrie et des bords du Rhin, qui sont bien supérieurs pour certains nsages aux aciers de cémentation. La présence du manganèse dans les minerais spatiques, comme ceux de Nastakopa, rend le fer malléable qu'on en obtient bieu plus propre à la fabrication do l'acier, et 'en Suède, quand le manganèse manqua, on y su,pplée par des mélanges manganésifêres qu'on introduit dazs le minerai de fer. Notre minerai de Nastukopa est donc de qualité supérieure sous ce rappont, puisqu'il contienc 24.64 pour 100 de carbonate manganésifère, d'après l'analyse qui en a été faite par le professeur Hoffman. Il est donc indubitable que ces mines renferment des richesses immenses, d'une valeur incalculable, que leur exploitation pourra se faire dans les conditions les plus faciles et les moins dispendieuses, que nous avons lat des minerais capables de fournir l'acier Bessmer de première qualité au monde entier, et que l'exploitation de ces mines contribuera puissamment a la colonisation de tonte la région avoisinante comme d celle du territoire de la baie James. Les mines de fer oligiste, de fer magnétique et de limonite de cette dernière région ont aussi une grande valeur; mais leur exploitation sera probablement retardée par celle des minerais spatiques de Nastakopa, qui offrent tant d'avantages et de si brillantes perspectives.

La yquène forme une couche d'une trentaine de pieds d'épaisseur et qui se prolonge sur le bord de la mer depuis l'embouchure de la petite rivière à la Baleine jusque dans le golfe Richmond, qui en est éloigné d'une trentaine de milles. Cette galène contient plus de 80 pour 100 de plomb, et deux échantillons analysés par le $\mathrm{Dr}$ Harrington ont donné respectivement 5.104 et 12.03 onces d'argent par tonneau de minerai de 2000 livres. Ces mines peuvent étre exploitées avec la plus grande facilité, et elles l'ont même été en 1858-9 par la compagnie de la baie d'Hadson, qui en a tiré nenf tonneaux de minerai pris dans quelques petites ouvertures, aus environs de la rivière aux Baleines. La quantité d'argent que l'on troure dans le minerai est suffisante pour payer une grande partie des frais d'exploitation de ces mines, qui ne sauraient manquer d'attirer la plus sérieuse altention des capitalistes.

Le manganèse, qui se trouve en si grande quantité dans le minerai de fer spatique de Nastakopa, est un minérai d'une grande utilité. On l'emploie dans la préparation clu chlore, et il en est importé plus de 30,000 tonneaux en Angleterre chaque année. Il constitue donc un objet d'exploitati.n d'une grande importance, et comme il passe dans les scories par la fusion du minerai de fer spatique, le traite. 
ment de ces minerais nous permettrait de produire lo mangan dese meilleuir marohé et en plus grande quantité que n'importe où ailleurs; dans des conditions partioulièrement avantagenses, puisque le minerai spatique permet de produire a la fois le fer et le manganèse, deux mótaux de la plus grande ntilité.

Les mines do cuivre da lac Abatngomaw renferment de grandes richesses qui pourront étre utilisées quand cette région sera traversée par un chomin de fer allant de Québeo d la baie James. Oes gisenesnts cupriferes couvrent une átendue de plusieurs milles carrés, ot l'extraction du minerai est d'autant plus facile et moins coûteuse que les roches cuivreuses formeut les couches superficielles du sol.

Depuis quelque temps, la compagnie du chemin de fer Canadien du Pacifique emploie pour chauffer ses locomotives du lignite provenant des mines de la rivière Souris. On dit que ce charbon donne plus de chaleur et moins de funée que la houille qu'on employait auparavant. Oeci serait de nature à donner de la valeur aux mines de lignite qu'on a découvertes dans les vallées do la rivièro Moose, de la rivière Missinaibi et do la rivière Albany. Co lignite, s'il faut en juger par les analyses da professeur Hoffman, est de qualité supérieure. Il y a des conches qui ont jusqu'à trois pieds d'épaisseur, et des recherches plus étendues, sinon plus soignées, que celles qui ont été faites jusqu'd ce jour, établiront que ces gisements de houille fossilifêre occupent une aire de plusieurs milliers de milles carrés: Lo charbon qu'on en tirera pourra être employé comme combustible sur les chemins de fer et surtout pour fondre les minerais de fer spatique de Nastakopa La principale raison qui s'oppose à l'emploi du lignite pour la fasion des minerais de fer, c'est le $E 0$ fre qui se trouve dans la cendre et qui pourrait se communiquer au métal fondu : or, nous a visns vu que cette objection disparalt, quand le minerai renferme du manganèse, qui absorbe le soufre dn combustible employé pour la fusion. Corame la fer spatique de Nastakopa renferme une forte proportion de manganèso, il pourra étre fondu sans inconvénient avec le lignito qui $\$ e^{\text {l }}$ rouve dane la région de la baio James, compa. rativement a peu do distance, en des endrrits qui permettent de faire les transports par la navigation, et par conséquent à très bon marché. Toutes ces circonstances réunips tendent a donner nne grando valeur à ces mines de for et d's charbon; en mèmo temps qu'elles en facilitent énormément l'exploitation.

Les antres minerais qui se trouvent dans cette région, notanment le plâtre, l'asbesto et lanthracite, fffrent aussi un champ d'exploitation qu'on ne manquera pas d'ut'lisor à mesure que le pays s'établira. On peut dire sans crainte que tout ce pays constitue l'une des plus belles et des plus riches régions minières du Canada, et méme de l'A mérique du Nord.

7. Le olimat, dans toute l'étenda que nous avons donnée comme susceptible do culture avantagense, est aussi bean et méme plus chaud, dans la région de la baie James, que dans la province de Manitoba et dans les prairies du Nord-Ouest. La saism agricole, 
c'est-d̂-dire la période exempte de gelées, est plus longue an fort Moose qu'd Winnipeg, plus longue que dans le district de Muskokn, et aussi longue que dans la plus grande partie do la province de Québec. Le fait que la flore des environs du fort Moose est la mème que celle des environs de Québec dérnontre clairement que le slimat doit être à peu près lo même dans ces deux losalités. A l'extrémits sad ue la baie Iames, la présence et la fonte des neiges retardont ui peu le printomps; mais en revanche la chaleur qui s'exhalo des ealux de la mer prolonge lo doux temps en antomne, de sorte que tout considéré. la belle saison est aussi longue que dans les régions do la rivière Rouge et plus longue que dans la partie nord-vuest de ln province d'Ontario. Le froid est un pen vif en hiver, comme dans le Nord-Ouest ; mais l'atmosphìre est si claire et si puro qu'on sapporto sans endurer lo moindre malaise les plus basses températures hibernales. Il tombe peu de pluie en été et peu de neige en hiver. Les rivieres commencent à charrier vers la fin d'avril ; lo dégel commenco dans la premièro quinzaine de mars, sous des températures qui s'élèvent souvent jusqu'à cinquante degrés; le sol se découvre au commencement de mai et peut être cultivé vors le miliea de ce mois. Les bourgeons des arbres sortent généralement entre le 12 et lo 15 mai, et la feuillaison est complète au $1^{\text {er }}$ juin, sonvent même avant cette date, dans les localités les plus favorisées sous le rapport de la température. Dans la région sud et sud-onest de la baie James, la première neige tombe généralernent à la fin d'octobre ou au commencemeit de noveunbre; la plus grando quantitó arrive avec lo noois de décembre, qui est ordinairement le plus neigeux et le plus froid. Durant les mois de janvier et février, le ternps est sec, clajr et très beat: Généralement parlant, le climat do la région de la baie James est pour lo moins aussi chand, et il est moins humide, moins brumeux que celui du district de Québec, ainsi que co fait est établi par les observations météorologiques qui ont ćté f'ites jusqu'd présent.

La preuve que le climat de ce vaste territoire est bien adapté à ia poursuite des opérntions agricol ss se troure dans les cultures qui se font deprais plus d'un siècle en plusieurs endroits de cette région. A l'exception du bié, on récolte partont les céréales et les légunes ordinaires aveo la plus grandé facilité. Les fermes de la compagnie do la baie d'Hudson à la rivière Rupert, au fort Moose, an fort NouveauBrunswick, à Osriaburg, les cultures faites par les particuliers an lace Oxford, an fort Norway, dans la vallée de la ririère aux Castors et sur les bords du lac de l'ile à la. Crosse, pronvent surabondamment ce fait. Sur plusieurs de ses fernes, même à celle de la rivière Rupert, la compaguie de la baie d'Hudson entretient constamment jusqu'ì quatre-vingts têtes de bêtes à comes, sans compter les moutons, les cochons et même des chevaux, à nu ou deux endroits. A moius qu'ils ne soient faits autrement que les antres, ces animaux doirent se nourrir de foin et de grains, et comme on n'en importe pas de l'étranger, il faut bien conclure que ce toin et ces grains sont récoltés sur les lieux mêmess, ce qui pronve d'uno hauière péremptoire que la 
oulture des céréales réussit bion partont, et que le climat n'est pas un obstacle d la culture.

An fort Moose, on récolto mémo la tomate, qui est une plautuexcessivement délicate, saus la cultivar avec plus do soin que dans les au. tres parties du Cauada. On re s'est jainai: beaucoup occupó de la calture du blé, parce que ce grain n'a guère d'ntilité à raison du petit. nombre de la population et surtout parce qu'on n'a pas do moulin pour le moudre couvenablement ; mais l'expérience qu'on a afite de cette culture dans la régiou de la rivière Moose et de la rivière Albany, a une cinquantaine de milles de la mer, prouve qu'elle réussit parfaitement. Mème an fort Moose, le blé d'automne a supporié les gelées d'hiver et très bien mûri au mois d'août suivant, quoi que cette localité soit environnée par un sol humide, froid, peu arantagoux, pins, dans le printemps, exposée aux vents froids de la mer et soumise d certaines inflencos climatériques défavorables qui ne se font pas sentir un peu plns loin dans l'intérieur des terres. Lo succès de la culture du blé dans la région ouest, c'est-à-diro dans la vallée de la rivière aux Castors ot sur les bords du lac de l'tle a la Crosse, cst attesté par Mgr Taché et le professeur Macoun, qui ont va de leurs yeux mèmes ce qu'ils raplortent.

Enfin, il est établi que io climat de cet immense territoire, sanf quelques variations locales et ancidentelles, est aussi bon, aussi chaud, aussi favorable aux opérations egricoles que le climat de la plus grande partie de la province de Québec, que celui do Muskoka et de la partie nord de la province d'Ontario.

$8^{\circ}$ La plus belle partie de ce territoire est la région $d u$ sud, on de la baie James, qui renferme au moins $108,800,000$ acres de très-bonnes terres arables, des foréts plus étendnes et presqu'aussi riches que celles de la provincé de Québec, des mines H $^{3}$ lignite, de plâtro et dè for d'une grande valeur, et qui pourra supporter et faire vivre dans l'aisance nue population d'au moins dix millions d'habitants, quand on la mettra en communication avec les grands centres de commerce, par la construction d'un chemin de fer.

La région de l'est a surtout do la valeur comme pays minier. C'est là que se trouvent les mines de fer spatique et de galène dont nous avons parlé ailleurs.

La région de l'ouest, surtout dans la vallée snpérieure do la rivière Nelson, renferme une quarantaine de millions d'acres de bonnes terres et des fortês capables de fonrnir beaucoup de bons bois à la consommatiou domestiquo comme à l'exportation. Cette région oceupe une excellente position cominerciale. O'est là que se concentreront les produits des prairies de l'ouest, si on réussit à construire le chemin de fer de la rivière Nelson et à établir une ligne de navigation maritime régulière entre le fort York et Liverpool, ce qui abrégerait de plusieurs centaines de milles la distance entre les riches plaines du Nord-Ouest et les ports anglais.

Il y a donc dans cette grande contrée, que nous désignons sous ie nom de bassin méridional de la baie d'Hudson, suffisumment de terres 
cultivables, an double point de rue de la dociceur du climat et de la bonne qualité du sol, pour établir nne population d'une trentaine de millions d'habitants, en supposant que les établissements se fassent, quant a l'étendue de chaque exfloitation, dans la même proportion que dans la partie labité de la province de Québec. Nous roulons bien admettre que cela ne se réalisera pas en entier d'ici a longtemps, et que les terres recouvertes de forêts de la région de la brie James n'attireront peut-être pas un très grand nombre de colons agricoles tant qu'on n'aura pas perdu un peu de l'engouement qu'on a contracté depuis ces dernières années pour les terres en prairie du Nord-Ouest. Mais cela n'empéche pas les faits d'exister ni la région do la baie James d'être ur excellent pays agricole, une contrée où les millions de pauvres gens qui vivent en Europe dans la znisère et le dénuoment pourraient sans peine se faire des établissements où ils vivraient dॄns l'aisance, le confort et la prospérité. Cela n'empêche pas non plus qu'il existe dans cette région des forêts et des mines d'une richesse incalculable, d'une exploitation on ne peut plus facile, plus lncrative, et si nous réussissons seulement a convaincre de ce fait quelques capitalistes entreprenants, nous aurons atteint en grande partie le but que nous avions en écrivant cetio esquisse. 
Observations meteorologiques fuites au fort York en 1876

\begin{tabular}{|c|c|c|c|c|c|c|c|c|c|c|c|c|c|c|c|c|c|c|c|}
\hline & $\ddot{\Xi}$ & & $a m b r$ & edes & 3 rent & ts a & 7a. $\mathrm{m}$ & $1.2 \mathrm{p}$ & $\mathrm{m} . \mathrm{c}$ & 0 & m. & & Pluie & & Nei & & 101 & $\dot{8}$ & \\
\hline & 高 & N. & N.E & E. & S. E & s. & S. 0 & 0 & N. 0 & 息 & 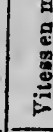 & $\stackrel{\stackrel{\Xi}{ت}}{:}$ & 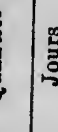 & & 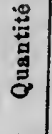 & 总 & $\mid \begin{array}{ll}0 & \\
0 & \\
0 & 0 \\
5 & 0 \\
\vdots \\
0 \\
0 \\
0\end{array}$ & 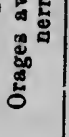 & \\
\hline ivier.. & 4 & & 0 & 8 & 5 & 4 & 17 & 7 & 23 & 17 & 74 & 0 & 00 & 0 & 113 & 20 & 0 & & \\
\hline rriar & 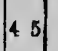 & 8 & 6 & 9 & 3 & 8 & 12 & 14 & 2 & 9 & 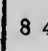 & 0 & 00 & & $\begin{array}{lll}6 & 5\end{array}$ & 14 & 0 & & \\
\hline 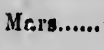 & $\mid \begin{array}{lll}3 & 1 \\
1\end{array}$ & & 15 & 0 & 0 & 8 & 6 & 9 & 23 & 15 & 58 & 0 & 00 & 0 & 67 & 11 & 0 & & \\
\hline ril ...... & 6.12 & & 13 & 13 & 10 & 10 & 3 & 11 & 6 & 5 & 9 & 0 & 04 & 1 & & 15 & 1 & & \\
\hline$i_{0}$ & 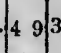 & & 24 & 7 & 2 & 10 & 9 & 5 & 3 & 1 & 78 & 0 & 25 & 1 & 10 & 4 & 1 & 1 & \\
\hline in ... & 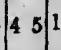 & & 15 & 19 & 14 & 18 & 2 & 10 & 9 & 1 & 91 & 7 & 70 & 7 & & & 1 & 1 & \\
\hline . & 15 & & 10 & 16 & 5 & 23 & 1 & 12 & 8 & 1 & 123 & 17 & $10 / 19$ & & & & 5 & 2 & \\
\hline & $\int^{4}$ & & 8 & 6 & 1 & 19 & 9 & 19 & 4 & 0 & 16 & 22 & 7011 & & & & 1 & 1 & \\
\hline$t \ldots \ldots$ & 69 & & 7 & 18 & 8 & 14 & 5 & 3 & 18 & 0 & 0 & 9 & 4011 & & i & & 1 & 3 & \\
\hline Phre & I 1 & & 12 & 10 & 0 & 13 & 0 & 4 & $29^{\circ}$ & 0 & 16 & & 97 & & & 18 & 7 & & \\
\hline &. & & 0 & 1 & 0 & $: 3$ & 12 & 12 & 24 & 0 & 12 & & 00 & 0 & $\left|\begin{array}{ll}20 & 1\end{array}\right|$ & 17 & 1 & & \\
\hline & 2 & & 0 & 0 & 0 & 20 & 20 & 17 & 13 & 0 & 17 & 0 & 00 & 0 & & 17 & 0 & & 11 \\
\hline & & & & 107 & 441 & 160 & 96 & 123 & 180 & 49 & & & & & & & 18 & 8 & \\
\hline
\end{tabular}

Pour les vents, ce iableau se repartit comme suit pour les quatre saisons :

\begin{tabular}{|c|c|c|c|c|c|c|c|c|c|c|c|c|}
\hline Saisons.......... & (N.) & & (N. E.) & (E.) & & (S. E.) & (S.) & & (\$.0) & (0.) & & $(N .0)$ \\
\hline Hiver............. & 46 & ....... & 6 & ...... 17 & ....... & 8 & ...... 32 & ....... & 49 & ...... 38 & $\ldots . .$. & 57 \\
\hline Printerups.... & 69 & $\ldots . .$. & 52 & ...... 20 & $\ldots . .$. & 12 & ..... 28 & ....... & i8 & .. 25 & & 49 \\
\hline Eté................. & 56 & ..... & 33 & ..... 41 & $\ldots . .$. & 20 & ...... 00 & ....... & 12 & $\cdots:$ & $\ldots . .$. & 21 \\
\hline e....... & 64 & $\ldots$ & 19 & $\ldots . . .29$ & ....... & 14 & 40 & ....... & 17 & $\ldots \quad 19$ & ....... & 71 \\
\hline Moyenne $0 l 0$ & 21.83 & & 10.40 & 10.10 & & 041 & 15.25 & & 7.15 & 11.72 & & 17.15 \\
\hline
\end{tabular}


Observations metcorologiques faites au fort Moose en 1879

\begin{tabular}{|c|c|c|c|c|c|c|c|c|c|c|c|c|c|c|c|c|c|c|}
\hline \multirow{2}{*}{ 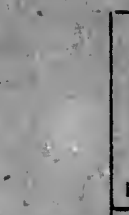 } & & \multicolumn{10}{|c|}{ Nombre des rents a $9 \mathrm{am}$ m., 2 p. m. ot 7 p. m. } & \multicolumn{2}{|c|}{ Pluie } & \multicolumn{2}{|c|}{ Noigo } & \multirow{2}{*}{ 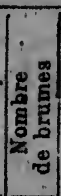 } & \multirow{2}{*}{ 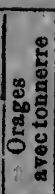 } & \multirow[b]{2}{*}{ 量 } \\
\hline & & $N:$ & N.E & E. & $\mid$ S. $\mathrm{E} \mid$ & s. & $\mid$ s. o $\mid$ & 0. & N.O & 曾 & 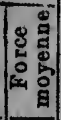 & $\mid \begin{array}{l}0 \\
\text { 量 } \\
\text { มี } \\
0\end{array}$ & 疍 & 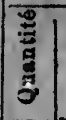 & 림 & & & \\
\hline & 6.3 & 5 & 3 & 5 & 1 & 7 & 26 & I4 & 24 & 8 & 2.1 & & 1 & 0.3 & 9 & & & 7 \\
\hline ier & 4.7 & 11 & 13 & 1 & 0 & 3 & 17 & 15 & 17 & 7 & 2.3 & 0 & 0 & 0.2 & 5 & & & 5 \\
\hline & 6.1 & 15 & 19 & 4 & 8 & 3 & 14: & 7 & 16 & 7 & 2.5 & $0.04 \mid$ & 1 & 0.8 & IS & & & 7 \\
\hline & 6.5 & 20 & 30 & 0 & 1 & 3 & 4 & 4 & 18 & 10 & 2.6 & 1.22 & 6 & 0.1 & 2 & 2 & to & 3 \\
\hline & 7.6 & 8 & 21 & 5 & 1 & 6 & 16 & 10 & 21 & 5 & 2.8 & 1.95 & 11 & 0.8 & 4 & 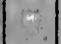 & & 4 \\
\hline & 8.3 & 27 & 28 & 1 & 8 & 3 & 13 & 0 & 13 & 3 & 3.0 & 1.64 & 9 & & 1 & 、 & 2 & 2 \\
\hline & 0.9 & 10 & 21 & 6 & 2 & 2 & 20 & 14 & 9 & 3 & 2.6 & 2.79 & 12 & & & 11 & 1 & 1 \\
\hline at & 7.0 & 19 & 14 & 0 & 2 & 1 & 23 & 16 & 12 & 6 & 2.1 & 6.11 & 17 & & 1 & 2 & 1 & 6 \\
\hline nt & 7.5 & 17 & 11 & 5 & 6 & 6 & 25 & 7 & 8 & 8 & 2.7 & 5.46 & 19 & & & 1 & 1 & 6 \\
\hline & 7.1 & 7 & 2 & 8 & 5 & 16 & 27 & 10 & 7. & 14 & 2.5 & 1.74 & 14 & 3.0 & 5 & & & 7 \\
\hline Nor & 7.9 & 9 & 5 & 1 & 0 & 8 & 14 & 12 & 29 & 12 & 2.5 & 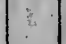 & 3 & 25.5 & 19 & & & 3. \\
\hline & 7.8 & 2 & 3 & 0 & 2 & 1 & 20 & 22. & 33 & 10 & 2.7 & & $1)$ & 27.4 & 23 & & & 3 \\
\hline & 6.6 & 156 & 170 & 33 & 30 & 59 & 219 & 131 & 204 & 93 & 2.5 & 2095 & 94 & 58.1 & 83 & 6 & 8 & 54 \\
\hline
\end{tabular}

Les vents du nord forment 15.56, cenx du sud-est 16.96, ceux do l'est 2.29 , ceux du sud-est 2.99 , ceux du sud 5.88 , ceux du sud-onest 21.84, ceux de l'ouest 13.07 et ceux du nord-ouest 20.35 pour 130 de la totalité de vents. Le groupe des vents du sui du sud-ouest et de l'ouest, qui sont des vents chauds, représente 1 . i seul 60.79 pour 100 , pour tonte.l'année, et pour les cinq mois de mai, juin, juillet, aout et septembre, qui constituent la saison agricole, la proportion de ces vents chauds est de 37.59 pour 100 , ce qui explique un pea la haute température d'été de cet endroit. Pour les quatre saisons, on trouve le tablean suivant .

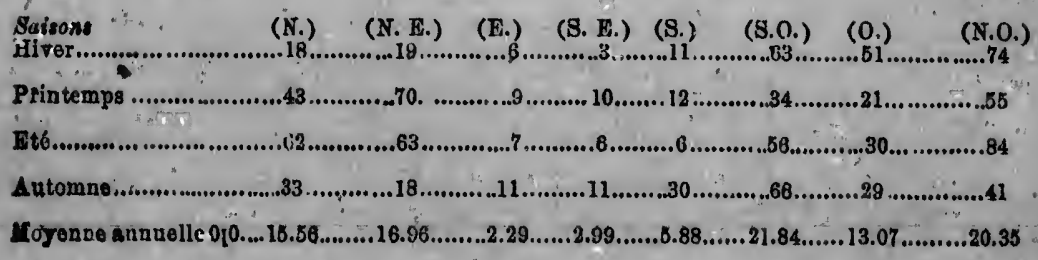





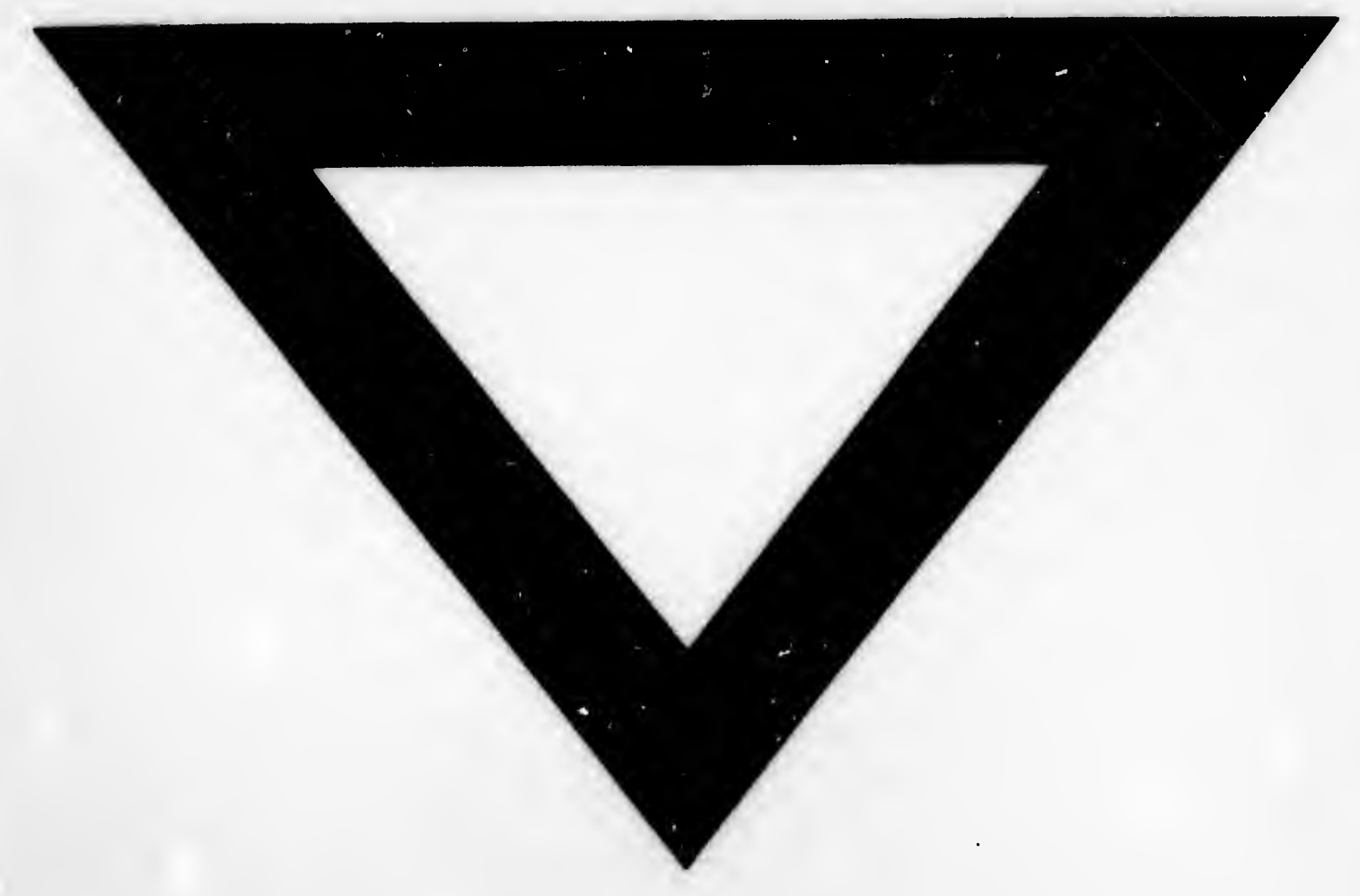

